\title{
Systematic Opacity Calculations for Kilonovae
}

\author{
Masaomi Tanaka, ${ }^{1 \star}$ Daiji Kato, ${ }^{2,3}$ Gediminas Gaigalas, ${ }^{4}$ Kyohei Kawaguchi ${ }^{5}$ \\ ${ }^{1}$ Astronomical Institute, Tohoku University, Sendai 980-8578, Japan \\ ${ }^{2}$ National Institute for Fusion Science, 322-6 Oroshi-cho, Toki 509-5292, Japan \\ ${ }^{3}$ Department of Advanced Energy Engineering Science, Kyushu University, Kasuga, Fukuoka 816-8580, Japan \\ ${ }^{4}$ Institute of Theoretical Physics and Astronomy, Vilnius University, Saulètekio Ave. 3, Vilnius, Lithuania \\ ${ }^{5}$ Institute for Cosmic Ray Research, The University of Tokyo, 5-1-5 Kashiwanoha, Kashiwa, Chiba 277-8582, Japan
}

4 June 2020

\begin{abstract}
Coalescence of neutron stars gives rise to kilonova, thermal emission powered by radioactive decays of freshly synthesized $r$-process nuclei. Although observational properties are largely affected by bound-bound opacities of $r$-process elements, available atomic data have been limited. In this paper, we study element-to-element variation of the opacities in the ejecta of neutron star mergers by performing systematic atomic structure calculations of $r$-process elements for the first time. We show that the distributions of energy levels tend to be higher as electron occupation increases for each electron shell due to the larger energy spacing caused by larger effects of spin-orbit and electron-electron interactions. As a result, elements with a fewer number of electrons in the outermost shells tend to give larger contributions to the bound-bound opacities. This implies that Fe is not representative for the opacities of light $r$-process elements. The average opacities for the mixture of $r$-process elements are found to be $\kappa \sim 20-30 \mathrm{~cm}^{2} \mathrm{~g}^{-1}$ for the electron fraction of $Y_{\mathrm{e}} \leq 0.20, \kappa \sim 3-5 \mathrm{~cm}^{2} \mathrm{~g}^{-1}$ for $Y_{\mathrm{e}}=0.25-0.35$, and $\kappa \sim 1 \mathrm{~cm}^{2} \mathrm{~g}^{-1}$ for $Y_{\mathrm{e}}=0.40$ at $T=5,000-10,000 \mathrm{~K}$, and they steeply decrease at lower temperature. We show that, even with the same abundance or $Y_{\mathrm{e}}$, the opacity in the ejecta changes with time by one order of magnitude from 1 to 10 days after the merger. Our radiative transfer simulations with the new opacity data confirm that ejecta with a high electron fraction $\left(Y_{\mathrm{e}} \gtrsim 0.25\right.$, with no lanthanide $)$ are needed to explain the early, blue emission in GW170817/AT2017gfo while lanthaniderich ejecta (with a mass fraction of lanthanides $\sim 5 \times 10^{-3}$ ) reproduce the long-lasting near-infrared emission.
\end{abstract}

Key words: radiative transfer - opacity — stars: neutron

\section{INTRODUCTION}

Coalescence of neutron stars (NSs) is a phenomenon of interest in a wide area in astrophysics: it is one of the primary targets of gravitational wave (GW) observations, a candidate progenitor of short gamma-ray bursts (GRBs), and a possible origin of the $r$-process elements in the Universe. In fact, the detection of gravitational waves from a NS merger has been achieved for the first time in 2017 (GW170817, Abbott et al. 2017a). Subsequent electromagnetic (EM) observations over a wide wavelength range (Abbott et al. 2017b) identified the counterpart AT2017gfo, and provided rich information including the link between NS mergers and GRBs (Abbott et al. 2017c) and $r$-process nucleosynthesis by the NS merger.

In particular, intensive observations have been

^ E-mail: masaomi.tanaka@astr.tohoku.ac.jp performed for AT2017gfo in the ultraviolet, optical, and infrared wavelengths (e.g., Andreoni et al. 2017; Arcavi et al. 2017; Chornock et al. 2017; Coulter et al. 2017; Cowperthwaite et al. 2017; Díaz et al. 2017; Drout et al. 2017; Evans et al. 2017; Kasliwal et al. 2017; Kilpatrick et al. 2017; Lipunov et al. 2017; McCully et al. 2017; Nicholl et al. 2017; Pian et al. 2017; Shappee et al. 2017; Siebert et al. 2017; Smartt et al. 2017; Soares-Santos et al. 2017; Tanvir et al. 2017; Tominaga et al. 2018; Troja et al. 2017; Utsumi et al. 2017; Valenti et al. 2017). The observed properties are broadly consistent with kilonova (Kasen et al. 2017; Tanaka et al. 2017; Perego et al. 2017; Rosswog et al. 2018, e.g., ), thermal emission powered by radioactive decays of newly synthesized $r$-process elements (Li \& Paczyński 1998; Kulkarni 2005; Metzger et al. 2010, see Rosswog 2015; Tanaka 2016; Fernández \& Metzger 2016; Metzger 2017 for reviews). The presence of the "red" (near-infrared, NIR) 
component implies that the ejecta are composed of lanthanide elements (Kasen et al. 2013; Tanaka \& Hotokezaka 2013). On the other hand, the "blue" (optical) component suggests that lighter $r$-process elements are also synthesized (Metzger \& Fernández 2014; Kasen et al. 2015; Tanaka et al. 2018). These multiple ejecta components are naturally expected in numerical simulations (see e.g., Shibata et al. 2017; Perego et al. 2017; Kawaguchi et al. 2018; Radice et al. 2018b; Shibata \& Hotokezaka 2019).

Although $r$-process nucleosynthesis is confirmed in GW170817/AT2017gfo, the exact abundance pattern synthesized by the NS merger is not yet clear. The most straightforward ways are identifying elements in the observed spectra and measuring their abundances. However, due to the large Doppler shift, blend of many absorption lines, and incompleteness of the atomic data, identification of the all the observed spectral features are challenging (see Watson et al. 2019, for the identification of Sr II lines).

Another method is modelling the light curves. For a simple one-zone model with a constant opacity, the typical peak time of the light curve scales as $t_{\text {peak }} \propto \kappa^{0.5}$, where $\kappa$ is the opacity. Accordingly, the peak luminosity scales as $L_{\text {peak }} \propto \kappa^{-0.65}$ if the radioactive decay luminosity decreases with $t^{-1.3}$, which is typical for neutron star mergers where $\beta$ decays of many $r$-process nuclei are involved (e.g., Metzger et al. 2010; Hotokezaka et al. 2017). Since $\kappa$ is sensitive to the element abundances (see below), we can indirectly infer the abundance from the light curves. In fact, many attempts of light curve modelling have been performed by assuming simple, constant opacities (e.g., Cowperthwaite et al. 2017; Villar et al. 2017; Perego et al. 2017). However, the opacities in the NS merger ejecta heavily depend on the wavelengths, and evolve with time by reflecting the changes in density, temperature, and thus, ionization/excitation states. Therefore, to connect the abundance patterns in the ejecta with the observed properties, we need to consider detailed atomic opacities of $r$-process elements

In fact, understanding of atomic opacities of $r$ process elements in kilonova has grown in the past several years. Kasen et al. (2013) first performed atomic structure calculations for selected lanthanide elements while Tanaka \& Hotokezaka (2013) compiled available data for $r$ process elements. They found that, as in the case of Fe-rich ejecta of Type Ia supernovae (Pinto \& Eastman 2000), the main contribution of the opacities come from bound-bound transitions of heavy elements, i.e., bound-free, free-free, and electron scattering opacitieis are subdominant. They also found high bound-bound opacities of lanthanide elements, which make kilonova fainter and redder than previously expected. Then, atomic structure calculations for selected lanthanide elements and lighter $r$-process elements have been performed by Fontes et al. (2017), Wollaeger et al. (2018), and Tanaka et al. (2018). More recently, Kasen et al. (2017) and Fontes et al. (2020) provided atomic calculations of all the lanthanide elements.

However, available atomic calculations still do not cover many $r$-process elements that NS mergers synthesize. Due to this situation, opacities of some representative elements have been used to compensate lacking data in detailed radiative transfer simulations. But the previous studies showed that, for example, the opacities of $\mathrm{Nd}$ and Er are different although they are both lanthanide elements (Tanaka et al.
2018; Fontes et al. 2020). Because of the lack of systematic atomic calculations, it has not been clear how large elementto-element variations of the opacities exist across the wide range of $r$-process elements, and how these variations affect the opacities of the NS merger ejecta.

In this paper, we perform the systematic opacity calculations of the $r$-process elements to understand the elemental variation of the opacities and physics behind it. As in the previous studies of kilonova opacity (e.g., Kasen et al. 2013; Fontes et al. 2017; Tanaka et al. 2018), providing very accurate atomic data (so-called spectroscopic accuracies, which are required for the opacities of stellar interior or stellar atmosphere), is beyond our scope since it is not yet computationally feasible (see Gaigalas et al. 2019; Radžiūtè et al. 2020, for efforts on selected elements). On the other hand, we aim at providing the complete dataset for the boundbound opacities of $r$-process elements. In Section 2, we show results of atomic structure calculations. We discuss elemental dependence of the opacities in Section 3. Then, we apply the opacity data for radiative transfer simulations in Section 4. Finally we give a summary in Section 5. Throughout of the paper, magnitudes are given in $\mathrm{AB}$ magnitude system.

\section{ATOMIC CALCULATIONS}

\section{$2.1 \quad$ Methods}

We perform systematic atomic calculations for the elements from $\mathrm{Fe}(Z=26)$ to $\mathrm{Ra}(Z=88)$. In this paper, we mainly focus on kilonova emission at $t \gtrsim 1$ day after the merger (hereafter $t$ denotes time after the merger). In such timescale, the temperature in the ejecta is $T \lesssim 20,000 \mathrm{~K}$, at which typical ionization stages of heavy elements are either neutral, or singly to triply ionized states (I - IV). We calculate atomic energy levels and radiative transitions of these ions using HULLAC (Hebrew University Lawrence Livermore Atomic Code, Bar-Shalom et al. 2001).

Since the calculation methods are same as in Tanaka et al. (2018), we give only a brief overview of the calculations. In the HULLAC code, the orbital functions are derived by solving the single electron Dirac equation with a central-field potential which includes both a nuclear field and a spherically averaged potential due to electronelectron interactions. Then, $N$-electron configuration state functions are constructed by coupled anti-symmetric products of the orbital functions. Relativistic configuration interaction (RCI) calculations are performed with the configuration state functions. In the RCI calculations, we include the ground-state and low excited-state configurations, which have a bulk contribution to the bound-bound opacity. The configurations used in our calculations are summarized in Table B1. The total Hamiltonian consisting of the DiracCoulomb Hamitonian, the Breit interaction and the leading QED corretions is diagonalized with the multi configuration state functions, and atomic energy levels are obtained as eigenvalues of the total Hamiltonian. Electric-dipole transition probabilities are calculated in length (Babushkin) gauge.

In the HULLAC code, the central-field potential is constructed from an electron charge distribution of the Slatertype orbital (see Equation (3) of Tanaka et al. 2018), for 


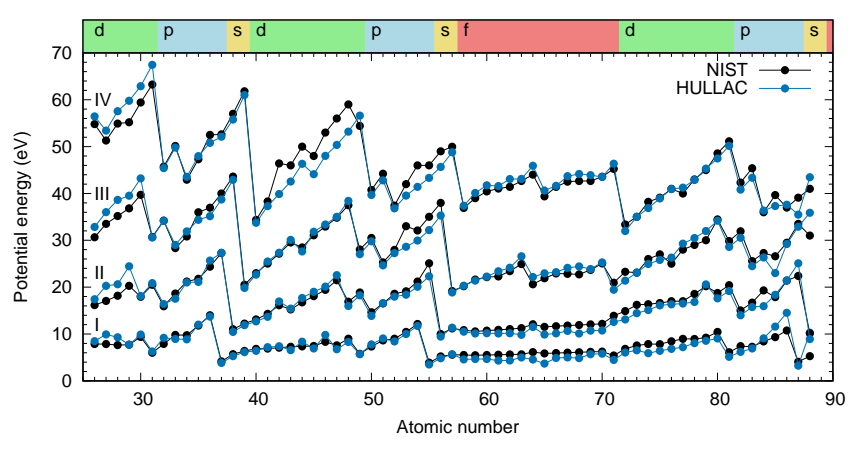

Figure 1. Comparison between the calculated ionization potentials using the HULLAC code (blue) and the those given in the NIST ASD (black, Kramida et al. 2018).
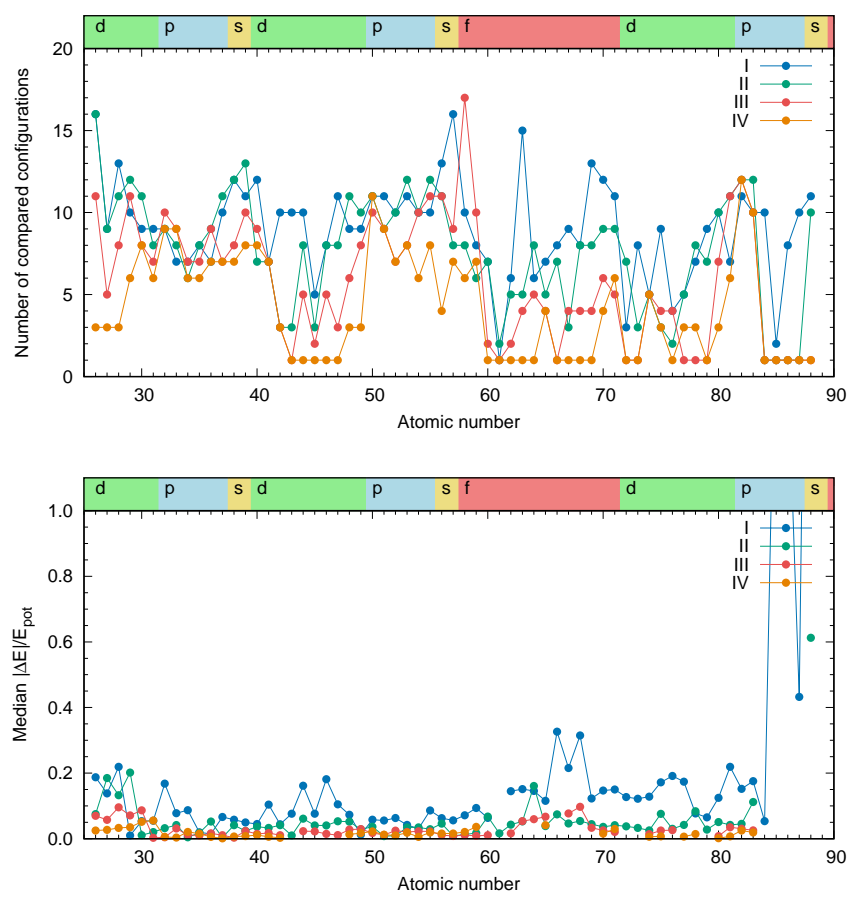

Figure 2. Comparison of the lowest energy of each configuration between our calculations and the NIST data (Kramida et al. 2018). Top: The number of configurations used for comparison. Bottom: Median of $|\Delta E| / E_{\mathrm{pot}}$, where $\Delta E$ is the difference in the lowest energy for a configuration between our calculations and the NIST data and $E_{\text {pot }}$ is the ionization potential.

which we use the ground state configuration of the next higher charge state. The potential is optimized so that the first-order configuration average energies of the ground state and low-lying excited states are minimized. The configurations used for the energy minimization are shown in bold in Table B1. To perform systematic calculations, we normally choose only the ground configuration for the energy minimization. However, we also include other configurations when the lowest energy for each configuration significantly deviates from that in the NIST Atomic Spectra Database (ASD, Kramida et al. 2018).
Since the calculations involve several assumptions as described above, we test the validity of our results by comparing the calculated ionization potential and derived energy levels with those in the NIST ASD. Although some uncertainties exist in the NIST data, this is the best possible way to evaluate our results. We do not include actinide elements because the energy levels are poorly known for most of them.

Figure 1 shows comparison of ionization potentials. Our atomic calculations give reasonable agreement, capturing the trend as a function of atomic number. In general, the agreement in higher ionization states is better, and the results of the neutral atoms shows the largest deviation in particular at high atomic numbers $(Z>60)$. The averaged fractional accuracies as compared with the NIST data are $14 \%, 7 \%, 4 \%$, and $4 \%$ for neutral atoms, singly, doubly, and triply ionized ions, respectively.

Overall agreement in the energy levels is similar to our previous results for selected elements (Tanaka et al. 2018). Some examples of energy levels are shown in Appendix A. Figure 2 shows typical accuracy of the lowest energy level for each configuration. For each configuration, we evaluate the difference in the lowest energy between our calculations and NIST data $(|\Delta E|)$. The lower panel shows the median of $|\Delta E|$ normalized by the ionization potential $\left(E_{\text {pot }}\right)$. The number of configuration used for the comparison is shown in the upper panel. As shown in the figure, a typical $|\Delta E| / E_{\text {pot }}$ is $<20$ $\%$ for neutral and $<10 \%$ for singly to triply ionized ions (except for $Z \geq 85$, see Section 2.2).

It is important to understand how accuracies in atomic calculations influence the bound-bound opacities. Since the NIST database only includes critically evaluated energy levels, the information of energy levels are not complete, and thus, we cannot compare the accuracies of all the energy levels. Therefore, it is not possible to directly evaluate the impact of the accuracy in atomic calculations to the opacities. Only the possible way is comparing the opacities calculated with different atomic codes or different assumptions in the atomic calculations. Kasen et al. (2013) shows that the difference in the strategy in the atomic calculations results in the difference in the opacity of $\mathrm{Nd}$ up to by a factor of 2. Tanaka et al. (2018) and Gaigalas et al. (2019) calculated the bound-bound opacities of selected $r$-process elements by using the HULLAC code and the GRASP2K code (Jönsson et al. 2013), which enables more ab-initio calculations without free parameters. They found that (1) overall wavelength dependence of the bound-bound opacities agree very well, (2) the maximum deviation is about a factor of 2 in ultraviolet wavelengths, and (3) the difference in the Planck mean opacities is up to a factor of 1.5. A similar agreement, within a factor of $1.5-2$, is also seen in the opacities of $\mathrm{Nd}$ II calculated by Kasen et al. (2013) and Fontes et al. (2017).

Therefore, we regard that a typical level of systematic uncertainty in the bound-bound opacity is about a factor of 2. As shown in the following sections, the elemental variation of the opacities is much larger than this uncertainty. This means that lack of data for some elements have a bigger impact to the opacity than the accuracy and systematics in the atomic calculations. 

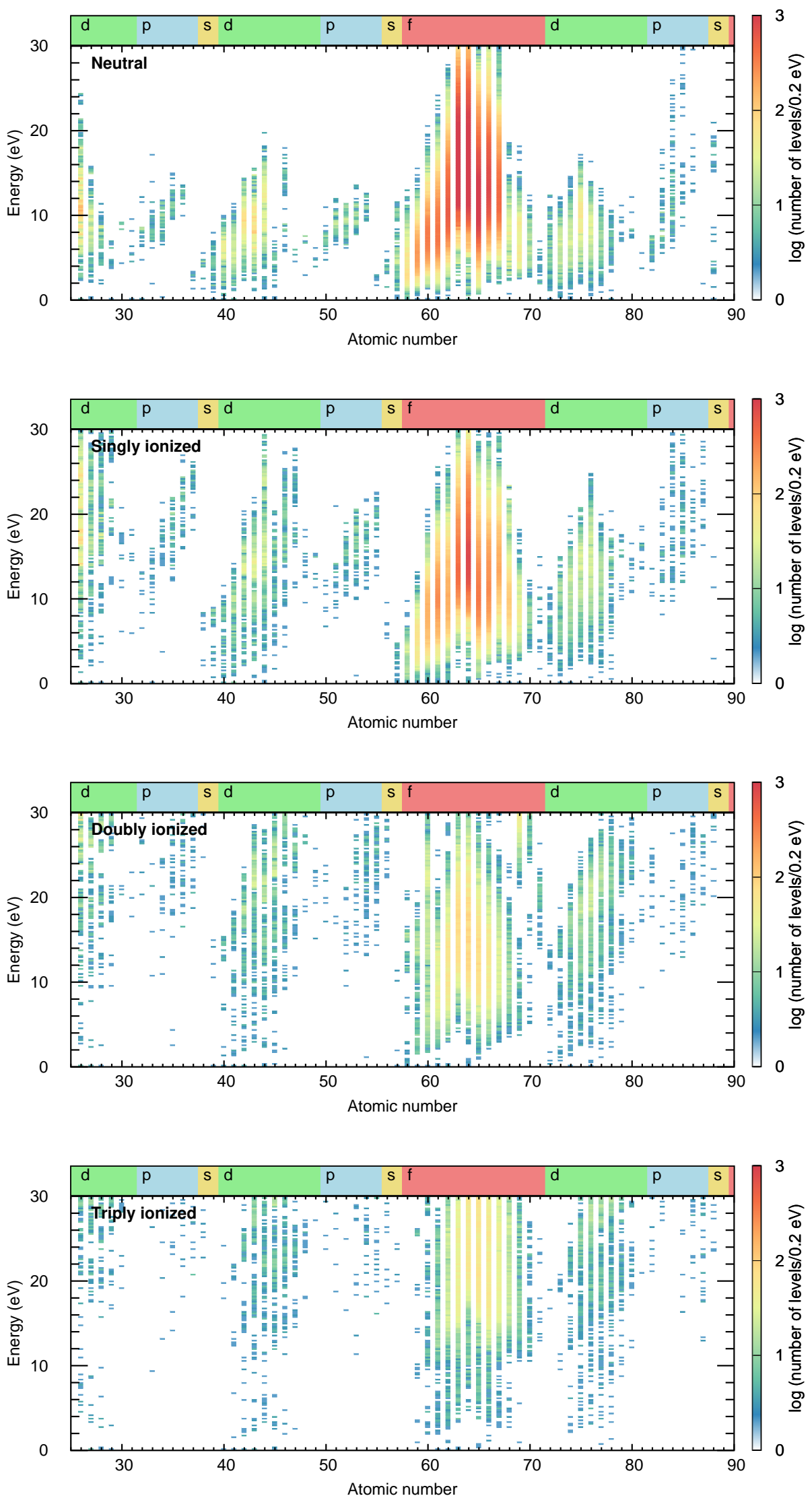

Figure 3. Distribution of energy levels of all the elements (neutral atom to triply ionized ion from top to bottom panels). The color scale represents the number of energy levels in $0.2 \mathrm{eV}$ energy bin. 


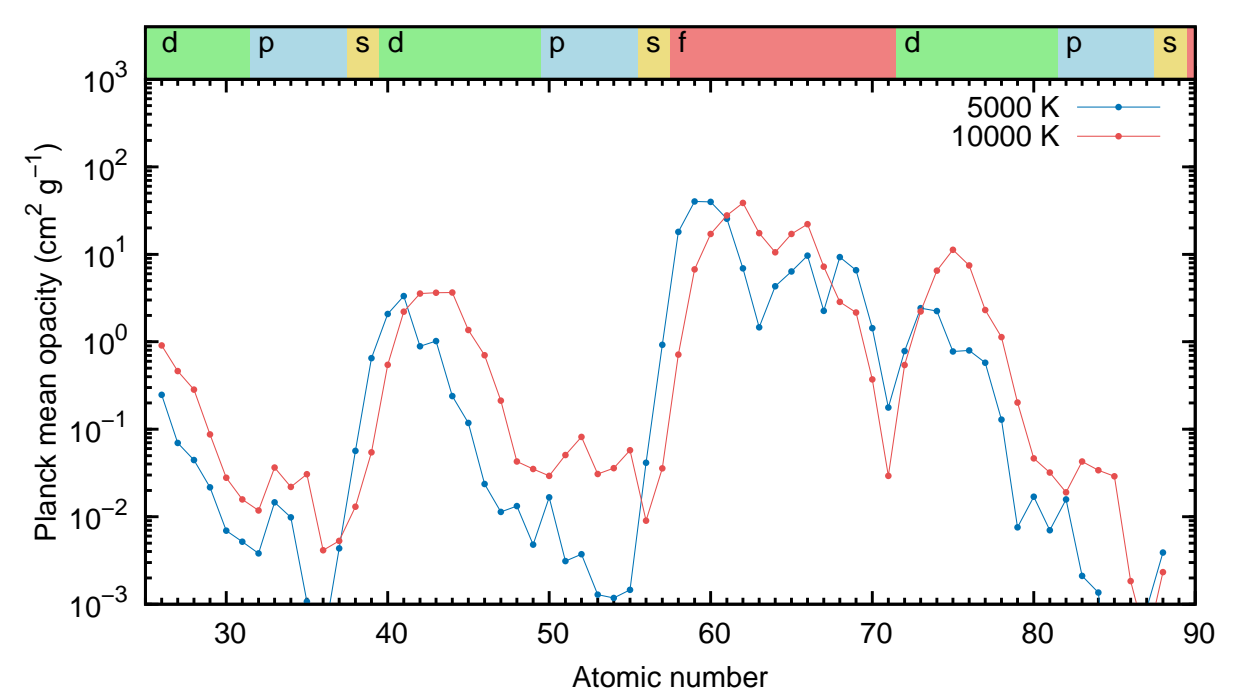

Figure 4. Planck mean opacities for all the elements. The opacities are calculated by assuming $\rho=1 \times 10^{-13} \mathrm{~g} \mathrm{~cm}^{-3}$, and $t=1$ day after the merger. Blue and red lines present the opacities for $T=5,000$ and 10,000 K, respectively.

\section{$2.2 \quad$ Energy levels}

Figure 3 summarizes the calculated energy levels for all the elements from $Z=26$ to $Z=88$. The color scale represents the distribution of energy levels, i.e., the number of energy levels in every $0.2 \mathrm{eV}$ energy bin. As expected from complexity measure (Kasen et al. 2013), $f$-shell elements have a larger number of energy levels than the other elements and then $d$-shell and $p$-shell elements follow.

Thanks to the systematic calculations for many elements, we identify the following two effects that mainly determine the energy level distribution. (1) Within a certain electron shell, the distribution of the energy levels tend to be shifted toward higher energy as more electrons occupy the shell (e.g., $Z=40-48$ for the case of $4 d$ shell and $Z=57-71$ for the case of $4 f$ shell). Since orbital radii become smaller with $Z$, Coulomb and spin-orbit integrals increase for higher $Z$, or in other words, electron-electron interaction and spin-orbit interaction energies become higher for higher $Z$. Therefore, the energy spacing, i.e., the energy difference to the neighboring level with the same parity and total angular momentum states, also increases along with $Z$ for a particular shell (see Figure 20-2 of Cowan 1981 for the case of $4 f$ shell). As a result, the distribution of the energy levels becomes wider for higher $Z$ for a given shell. (2) At the same time, the number of states is the largest for the half-closed shell since it gives the highest complexity, i.e., the number of combinations formed from different quantum numbers is the largest.

The latter effect is strong in lanthanide elements $(Z=$ $57-71)$. The total number of levels is the largest for elements around $\mathrm{Eu}$ or $\mathrm{Gd}$ which have half closed $4 f$-shells. But the distribution of the energy levels is pushed up for these elements, and thus, the number of low-lying levels is not necessarily higher than that of other lanthanide elements. This is the reason why the bound-bound opacities of these complex elements are not always higher than those of the other lanthanides (see Section 3). In addition, due to the former effect, the energy distributions of the elements with the conjugate configurations are wider for higher $Z$. For example, Dy I ( $Z=66$, with the ground configuration of $\left.4 f^{10} 6 s^{1}\right)$ has a wider energy distribution than the conjugate Nd I $\left(Z=60,4 f^{4} 6 s^{1}\right)$. By this effect, $\mathrm{Nd}$ I tends to have higher bound-bound opacities than Dy I, in particular for a low temperature (see Section 3).

In Figure 2, the elements with $Z \geq 85$ show large deviations from the NIST data. For these elements, configuration energies for $6 \mathrm{~s}, 6 \mathrm{p}$ and $7 \mathrm{~s}$ electrons are found to be pushed up because electrons in these orbitals are too much compressed in a small region, and feel a strong electron-electron repulsion. The repulsive interaction is effectively reduced by including configuration mixing with outer orbitals, but the overall energy level distribution tends to be extended to higher energy. Also, by the presence of the strong mixing configuration, the label of the energy level is not clear, which makes direct comparison with the NIST data difficult. Due to these facts, the opacities of these heavy elements can be more uncertain than those of the other elements. We discuss the impact to the opacities in the following sections.

\section{BOUND-BOUND OPACITY}

In a typical timescale of kilonova emission $(t>1$ day), bound-bound transitions of heavy elements is the dominant source for the opacities in near ultraviolet, optical, and infrared wavelengths (Kasen et al. 2013; Tanaka \& Hotokezaka 2013). Bound-free and free-free transitions and electron scattering give only minor contributions, although they are included in the radiative transfer simulations shown in Section 4. In the ejecta of NS merger or supernovae, Sobolev approximation (Sobolev 1960) can be applied as a large velocity gradient exists and the thermal line width $\left(\sim 1 \mathrm{~km} \mathrm{~s}^{-1}\right)$ is negligible compared with the expansion velocity (Kasen et al. 2013). The optical depth of one bound-bound transition can be expressed by Sobolev 
optical depth $\tau_{l}$ :

$\tau_{l}=\frac{\pi e^{2}}{m_{e} c} f_{l} n_{i, j, k} t \lambda_{l}$

for homologously expanding material $(d r / d v=t)$. Here $n$ is the population of a lower level of the transition $(i$-th element, $j$-th ionization stage, and $k$-th excited level) and $f_{l}$ and $\lambda_{l}$ are the oscillator strength and transition wavelength, respectively. The Sobolev approximation cannot be used when the wavelength spacing of the strong lines becomes comparable to the thermal width. We confirmed that, in the typical condition for kilonova, the wavelength spacing is larger than the thermal width by a factor of $>10$, and thus, the Sobolev approximation is applicable (see Appendix B).

To evaluate the Sobolev optical depth, we need ionization and excitation $\left(n_{i, j, k}\right)$. Our calculations assume local thermodynamic equilibrium (LTE), and ionization states $(j)$ are calculated by solving Saha equation. Population of excited states follow the Boltzmann distribution, i.e., $n_{i, j, k} / n_{i, j, 0}=\left(g_{k} / g_{0}\right) \exp \left(-E_{k} / k T\right)$, where $g_{0}$ is the statistical weight of the ground state and $g_{k}$ and $E_{k}$ are the statistical weight and energy of an excited level $k$. By this exponential dependence of the population of excited states, boundbound transitions from lower energy levels have much higher contributions to the total opacities.

To compute the bound-bound opacity for a certain wavelength grid $\Delta \lambda$, we adopt expansion opacity formalism, which is commonly used for supernovae and NS mergers (Karp et al. 1977; Eastman \& Pinto 1993; Kasen et al. 2006). The expansion opacity for the homologously expanding material is written as follows:

$\kappa_{\exp }(\lambda)=\frac{1}{c t \rho} \sum_{l} \frac{\lambda_{l}}{\Delta \lambda}\left(1-e^{-\tau_{l}}\right)$,

where summation is taken over all the transitions within the wavelength bin $\Delta \lambda$ in radiative transfer simulations.

Since the summation is calculated for all the calculated transitions, the expansion opacities can depend on the number of calculated transitions. The number of transitions is limited by the number of configurations included in the atomic structure calculations. In other words, too few configurations in atomic structure calculations can result in the underestimation of the bound-bound opacities. To study the convergence in terms of the number of included configurations, we calculate the opacities by adding configurations one by one. We confirm that our choice of configurations give a convergence in the opacities within $10 \%$. This is smaller than the expected systematic uncertainty of the opacity caused by assumptions in the atomic calculations (by a factor of 1.5-2, see Section 2). The details of this convergence studies are given in Appendix A.

In this paper, whenever not explicitly mentioned, the expansion opacities are evaluated at $t=1$ day after the merger by assuming density of $\rho=1 \times 10^{-13} \mathrm{~g} \mathrm{~cm}^{-3}$, which is typical for the ejecta mass of $M_{\mathrm{ej}} \sim 10^{-2} M_{\odot}$ and the ejecta velocity of $v \sim 0.1 c$. In this section, we show the opacity for each element i.e., the opacity is computed by assuming gas purely consisting of one element to study the elemental variation of the opacity. In Section 4, we show the opacities for the mixture of $r$-process elements.

Figure 4 shows the overview of the opacity as a function of atomic number: the Plank mean opacities are shown for $T=5,000$ and $10,000 \mathrm{~K}$ for all the calculated elements. The elemental variation is significant, ranging from $\kappa \sim 10^{-3} \mathrm{~cm}^{2} \mathrm{~g}^{-1}$ to $\kappa \sim 50 \mathrm{~cm}^{2} \mathrm{~g}^{-1}$. A notable feature is that the variation is quite large even for the elements with the same outermost electron shell. In the following sections, properties of the opacities and physics behind the behaviors are discussed for the elements with each outermost shell.

\section{1 f-shell elements}

Open $f$-shell elements (lanthanides) have larger opacities than the elements with other outermost electron shells (Kasen et al. 2013; Tanaka \& Hotokezaka 2013; Fontes et al. 2017; Tanaka et al. 2018; Wollaeger et al. 2018; Fontes et al. 2020). Due to the large number of energy levels with small energy spacing, the opacities remain high in the NIR wavelengths (left panels of Figure 5). Depending on the elements and temperature, the Planck mean opacities are $\kappa \sim 0.1-50 \mathrm{~cm}^{2} \mathrm{~g}^{-1}$ (right panels).

For $T=5,000 \mathrm{~K}$, Planck mean opacities of $\mathrm{Pr}, \mathrm{Nd}$, and $\operatorname{Pm}(Z=59,60$, and 61$)$ are the highest among lanthanide elements (Figure 6). The opacities gradually decrease as more electrons occupy $4 f$-shell. This is because the number of lowlying energy levels decreases as $f$-shell has more electrons (i.e., $Z$ increases). Although the total number of energy levels is the largest for nearly half-closed $f$-shell elements (Eu or $\mathrm{Gd}$ ), their opacities are not necessarily highest, as also found by Kasen et al. (2017) and Fontes et al. (2020). This is understood by the relatively high energy level distributions of Eu and Gd (Figure 3).

For $T>10,000 \mathrm{~K}$, the Planck mean opacities are the highest for nearly half-closed elements (Figure 6). This is because high excited levels of Eu or Gd start to contribute to the opacities. Also, at this temperature, the lanthanides are doubly ionized and low- $Z$ lanthanide elements such as $\mathrm{Pr}$ and $\mathrm{Nd}$ have smaller contributions to the opacities.

Temperature dependence is different for low and high electron occupations in $f$-shell (Figure 6). This dependence is more clearly visible in the right panels of Figure 5 . Low- $Z$ lanthanide elements such as $\mathrm{Ce}, \operatorname{Pr}, \mathrm{Nd}(Z=58,59$, and 60) show decreasing Planck mean opacities as a function of temperature because they have smaller number of electrons in $4 f$-shell. On the other hand, elements with more $f$-shell electrons such as Sm, Eu, Gd, Tb, Dy, Ho, Er, Tm, and Yb $(Z=62-70)$ show increasing opacities with temperature since they become closer to half-closed shell as temperature increases.

As shown in the right panels of Figure 5, our opacity data for $f$-shell elements are applicable only at $T \lesssim 20,000$ $\mathrm{K}$ since our atomic calculations include only up to triply ionized ions. This temperature corresponds to about $0.5-1$ day after the merger although this epoch depends on the ejecta parameters such as mass, velocity, and opacity. We need atomic calculations for highly ionized ions to correctly understand the emission at earlier epochs.

\section{2 d-shell elements}

Open $d$-shell elements have the second largest contributions to the opacities after open $f$-shell elements. Compared with the $f$-shell elements, the opacities of the $d$-shell elements 

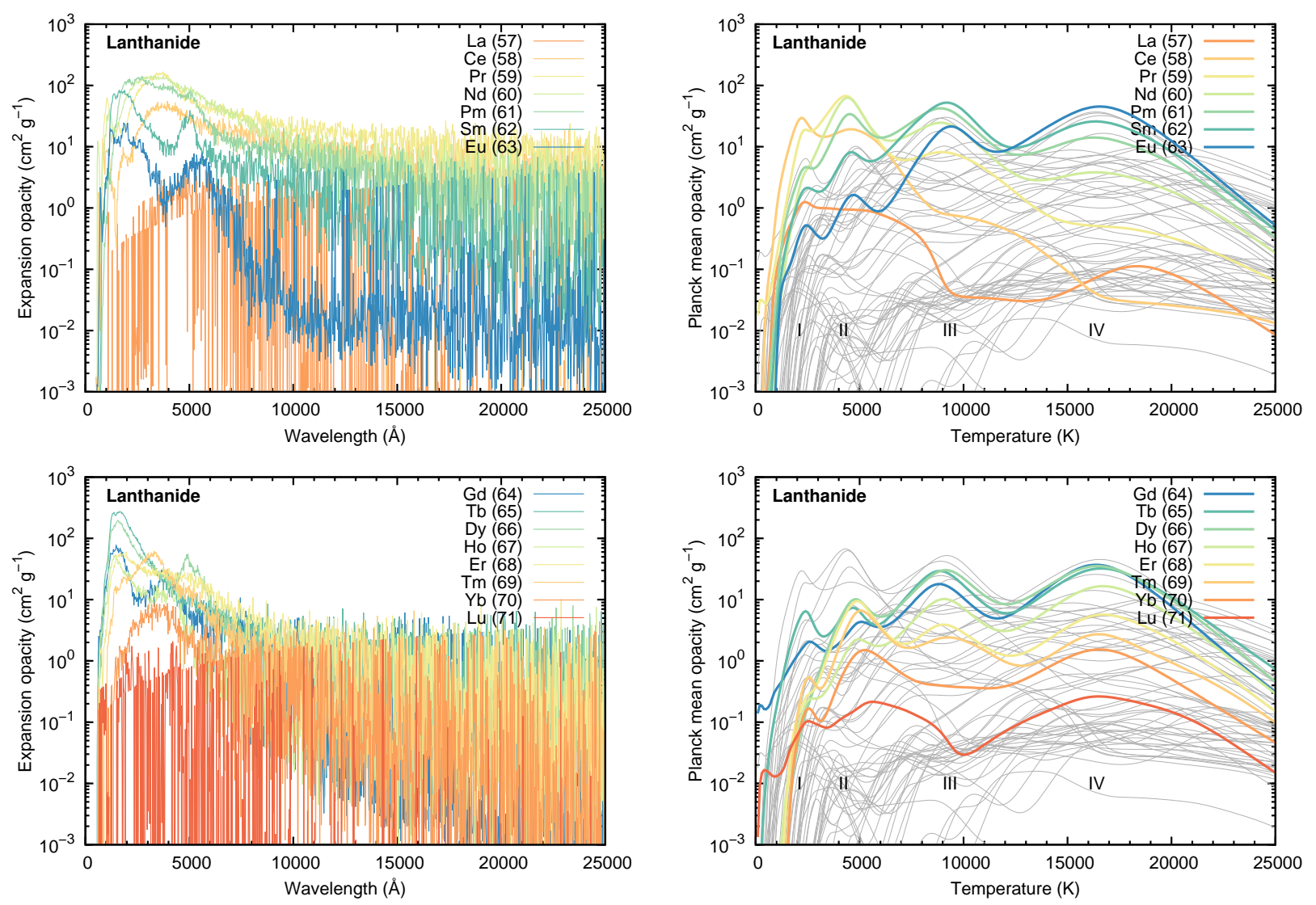

Figure 5. Left: Expansion opacity for $f$-shell (lanthanide) elements at $T=5,000 \mathrm{~K}$. Right: Planck mean opacities as a function of temperature (color). Gray lines show the Planck mean opacities of all the other elements. The labels (I, II, III, and IV) show typical temperature ranges for each ionization state.

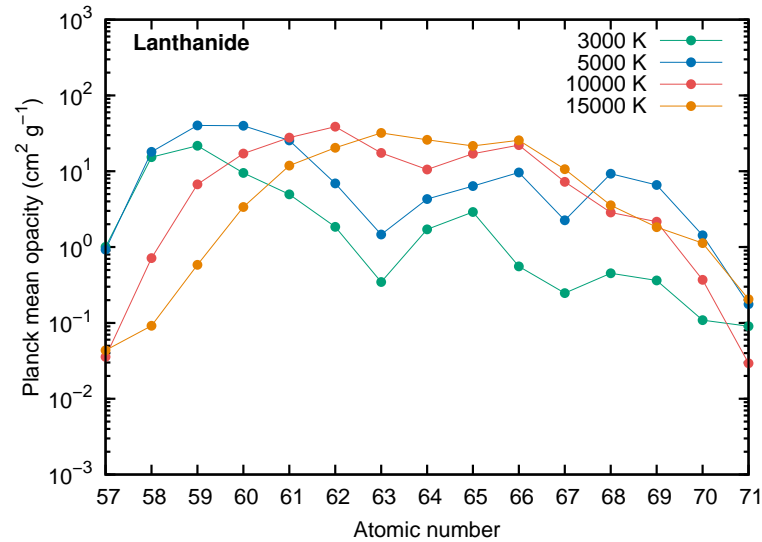

Figure 6. Planck mean opacities of lanthanide elements as a function of atomic number. For lower temperature $(T<5,000$ $\mathrm{K})$, the opacity tends to decrease for higher atomic numbers. For higher temperature $(T>10,000 \mathrm{~K})$, the opacities are highest around half-closed elements.

have a stronger wavelength dependence, i.e., the opacities are more concentrated to the shorter wavelengths around $1,000-3,000 \AA$ (left panels of Figure 7). The Planck mean opacities are within the range of $\kappa \sim 0.01-10 \mathrm{~cm}^{2} \mathrm{~g}^{-1}$ (right panels).

For relatively low temperature $(T<5,000 \mathrm{~K})$, the el- ements with a smaller number of $d$-shell electrons tend to have larger opacities (Figure 8). This is due to the lower energy level distributions and larger number of active strong transitions for the elements with the smaller number of $d$ shell electrons (Figure 3). For a higher temperature, the contributions to the opacities from the elements with 1 or 2 electrons in neutral atoms ( $\mathrm{Zr}$ and $\mathrm{Nb}$ for $4 d$, $\mathrm{Hf}$ and $\mathrm{Ta}$ for $5 d$ ) becomes smaller (right panels in Figure 7) since these elements do not have $d$-shell electrons when doubly ionized. This is the reason why the Planck mean opacities have a peak around groups 7 and 8 at $T=10,000 \mathrm{~K}$.

As in the case of $f$-shell elements, opacities are underestimated at a high temperature $(T \gtrsim 20,000 \mathrm{~K})$ due to the lack of atomic data of higher ionization states. The applicable temperature range for $d$-shell elements is wider than that of $f$-shell elements because of the higher ionization potential of the $d$-shell elements.

\section{$3.3 \quad$ p-shell elements}

Open $p$-shell elements have smaller contributions to the opacities compared with open $d$-shell and $f$-shell elements (Kasen et al. 2013; Tanaka et al. 2018; Wollaeger et al. 2018). The opacities are highest at ultraviolet wavelengths (left panels of Figure 9). For the optical and near-infrared wavelengths, the Planck mean opacities increase as a func- 

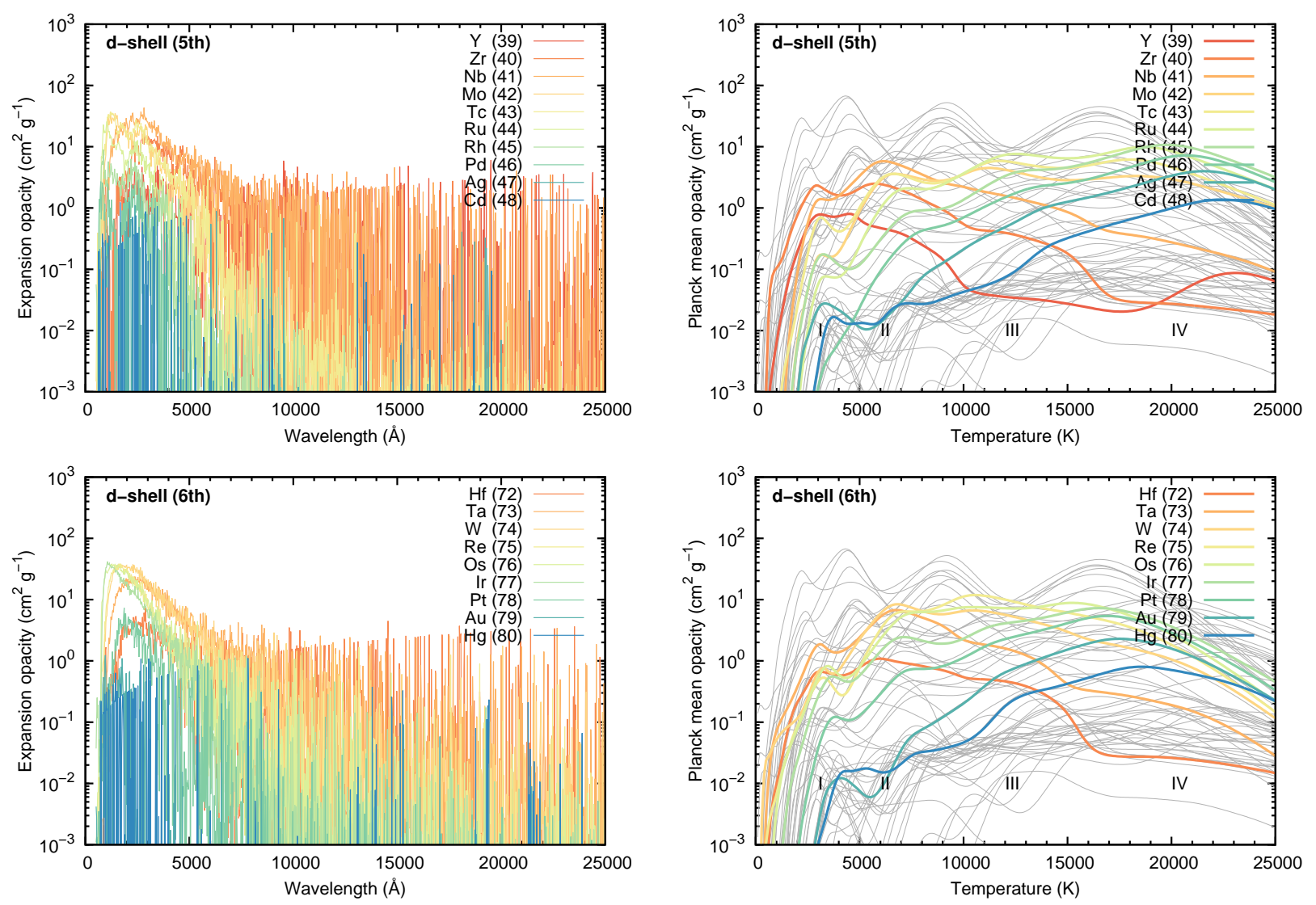

Figure 7. Same as Figure 5 but for $d$-shell elements.

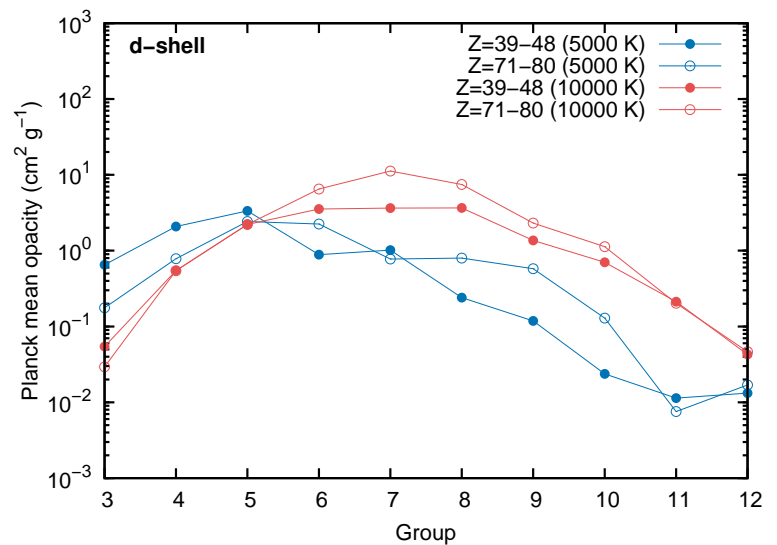

Figure 8. Planck mean opacities of $d$-shell elements $(Z=39-48$ in the 5th period and $Z=71-80$ in the 6 th period) as a function group in the periodic table (the group number is approximately the number of electrons in the $d$-shell for the case of neutral atoms).

tion of temperature but they are at most $\kappa \sim 1 \mathrm{~cm}^{2} \mathrm{~g}^{-1}$ for $T<20,000 \mathrm{~K}$ (right panels).

As in the cases for open $d$-shell elements, the opacities of $p$-shell elements are smaller for more $p$-shell electrons (Figure 4) since the distribution of energy levels is shifted toward higher energy. This trend is more significant because the average energy levels of $p$-shell elements are higher than those of $d$-shell elements (Figure 3 ).
As discussed in Section 2, the elements with $Z \geq 85$ show large deviation in the energy level as compared with the NIST data. The energy levels of these elements tend to be pushed up. As a result, the opacities of these elements are significantly underestimated. At $T<5000 \mathrm{~K}$, the Planck mean opacity of At $(Z=85)$ becomes lower than that of I $(Z=53)$ by a factor of 100 . The effect is even bigger for $\mathrm{Rn}(Z=86)$ : the opacity of $\mathrm{Rn}$ is lower than that of Xe $(Z=54)$ by a factor of 100 for a wide temperature range. Therefore, we regard that the opacities of these elements are not reliable. Note that the contribution of these elements to the total opacity in the NS merger ejecta is quite small (see Section 4).

\section{$3.4 \quad$ s-shell elements}

The opacities of open $s$-shell elements are almost negligible to the total opacities. Since there are fewer number of transitions, they do not form quasi-continuum opacities (left panel of Figure 10). For the typical temperature of kilonovae, the Planck mean opacities are $\kappa \lesssim 0.1 \mathrm{~cm}^{2} \mathrm{~g}^{-1}$ (right panel). Note that, as for the case of $\tilde{\text { At }}(Z=85)$ and $R n$ $(Z=86)$, our opacities for $\operatorname{Fr}(Z=87)$ and $\operatorname{Ra}(Z=88)$ are not reliable. Although the opacity of Fr shows similar trend with $\mathrm{Rb}(Z=37)$, the opacity of $\mathrm{Ra}$ is significantly lower than those of the other $s$-shell elements.

Overall low opacities of $s$-shell elements do not necessarily mean that they do not contribute to the outcome of kilonova emission. In fact, open $s$-shell elements such as $\mathrm{Na}$, 

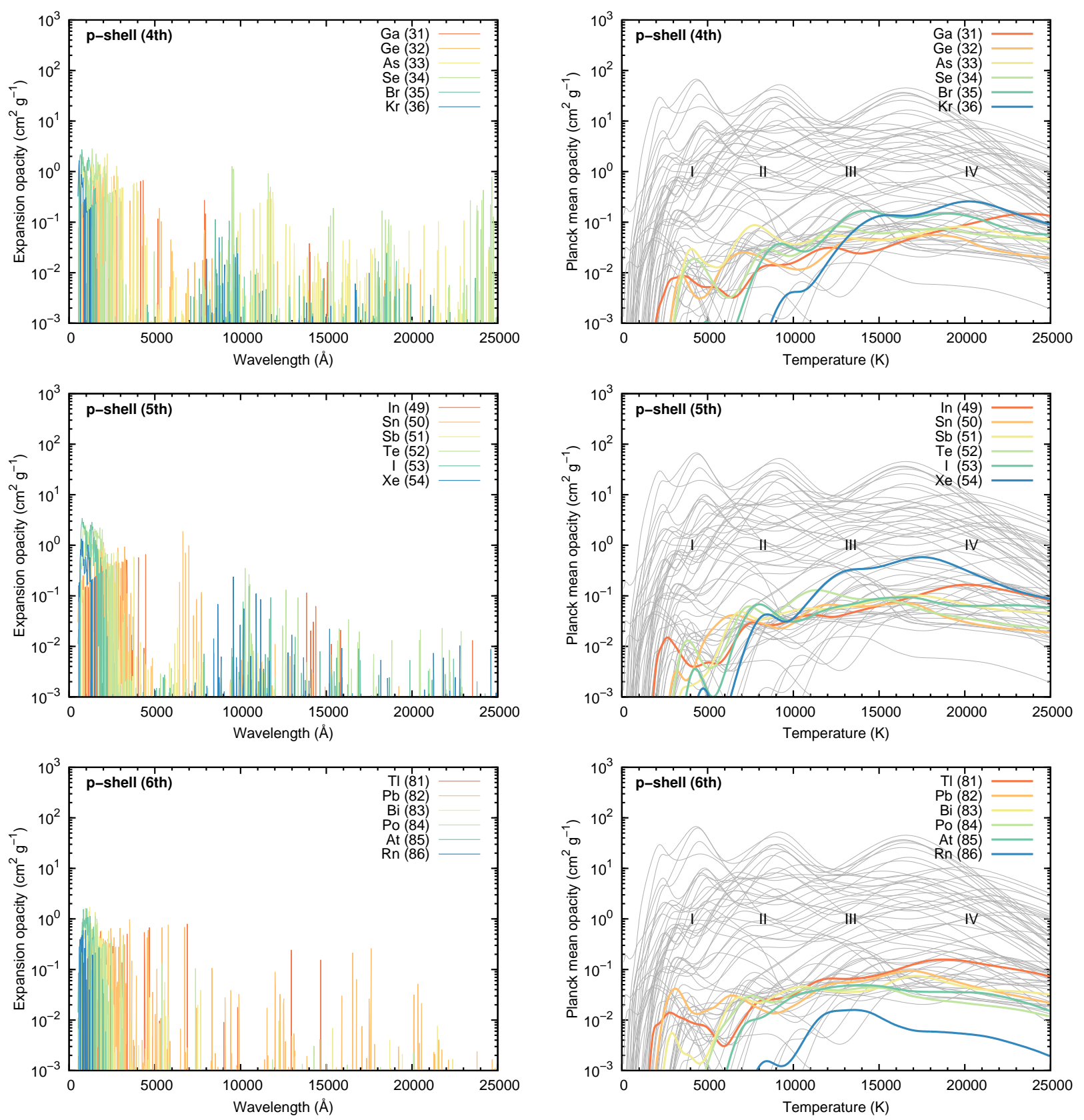

Figure 9. Same as Figure 5 but for $p$-shell elements.

$\mathrm{Mg}$, and $\mathrm{Ca}$ often show strong absorption lines in stellar spectra. and thus, $s$-shell elements may contribute to absorption lines in the spectra (see Watson et al. 2019 for the identification of $\mathrm{Sr}$ in the spectra of AT2017gfo). Unfortunately, since the atomic calculations for $r$-process elements do not give spectroscopic accuracy, i.e., high enough accuracy to predict the exact wavelengths of each transition, the usefulness of our opacity data for open $s$-shell elements is limited.

\section{APPLICATIONS TO KILONOVAE}

\subsection{Opacities for the mixture of the elements}

Ejecta from NS mergers consist of mixture of $r$-process elements. Abundance distribution is mainly determined by electron fraction $Y_{\mathrm{e}}$. The first dynamical ejecta have a wide $Y_{\mathrm{e}}$ distribution down to $Y_{\mathrm{e}} \sim 0.1$ (Wanajo et al. 2014; Sekiguchi et al. 2015, 2016; Goriely et al. 2015; Radice et al. 2016; Foucart et al. 2016) while subsequent post-merger ejecta can have higher $Y_{\mathrm{e}}$ due to the neutrino absorption if a massive neutron star remains for longer than a certain period (Fernández \& Metzger 2013; Fujibayashi et al. 2018; Radice et al. 2018a; Fernández et al. 2019). Lanthanide elements are efficiently produced with $Y_{\mathrm{e}} \lesssim 0.25$ (e.g., 

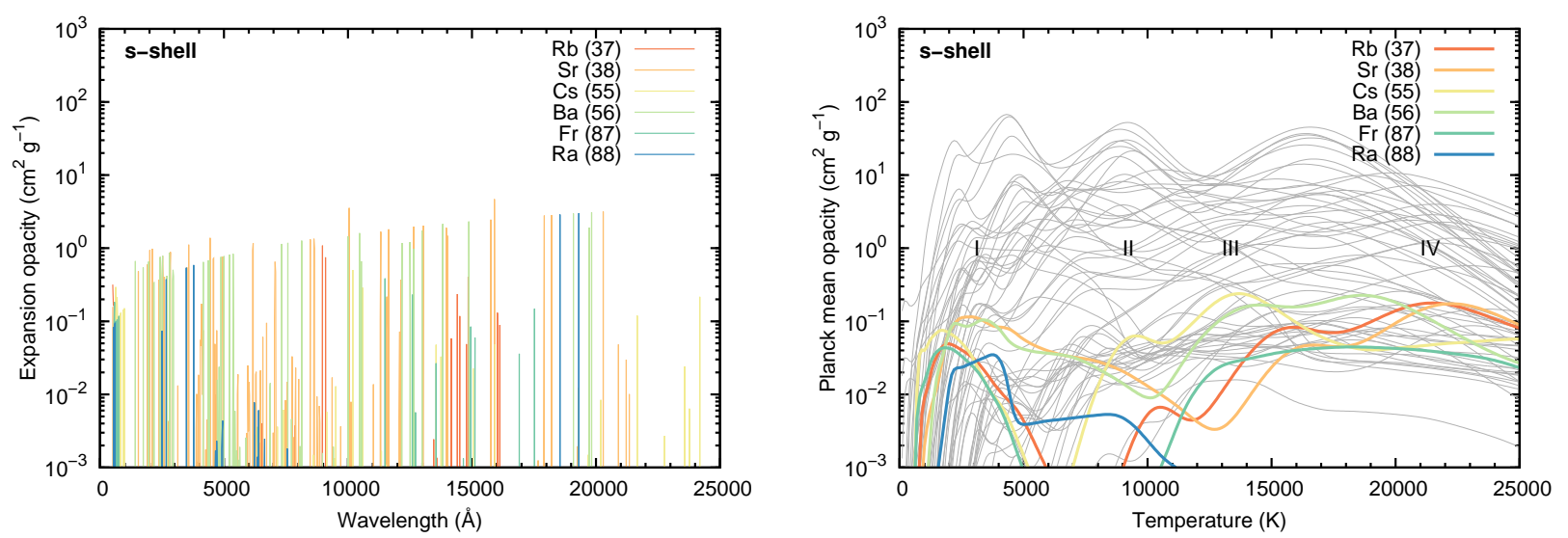

Figure 10. Same as Figure 5 but for $s$-shell elements.
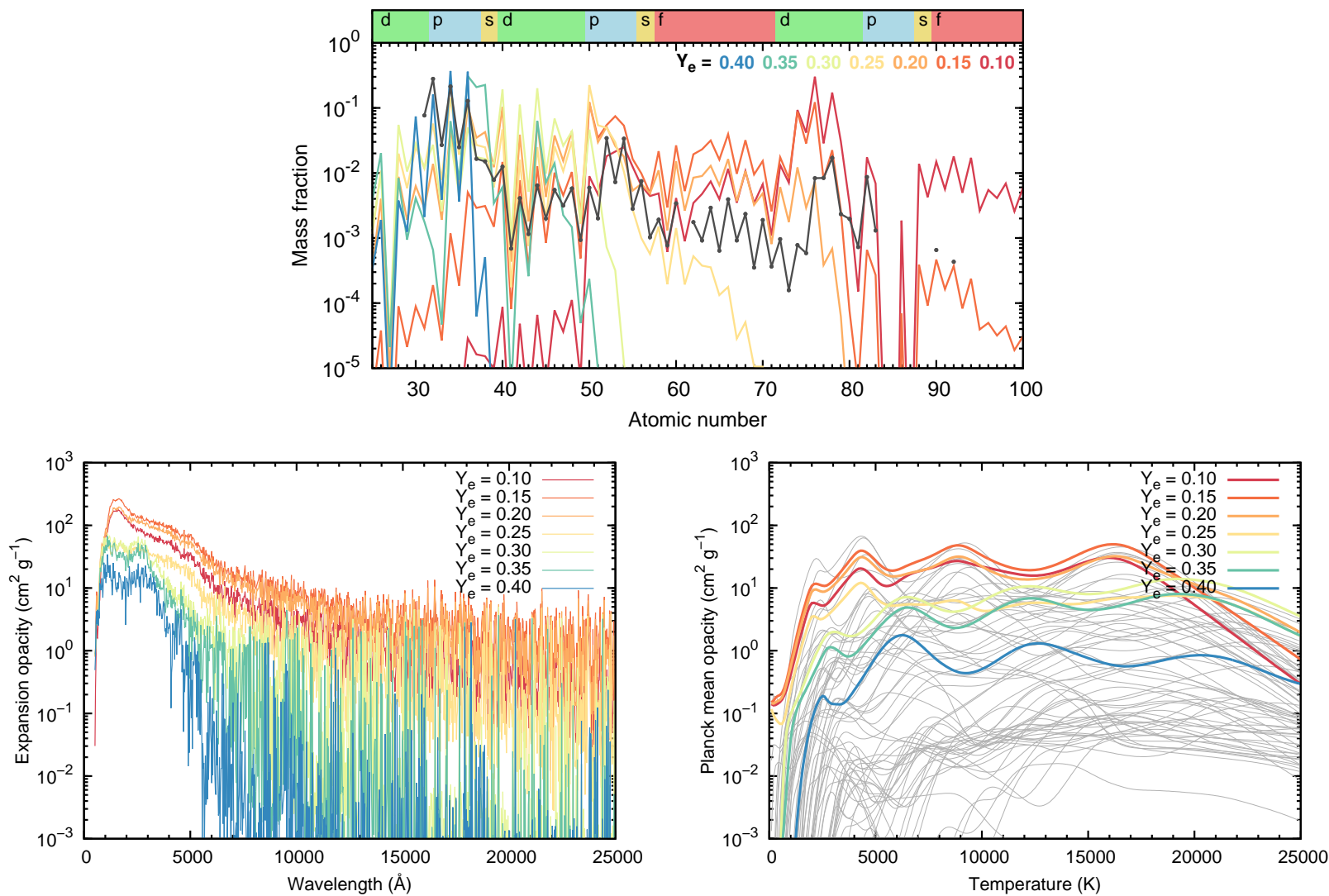

Figure 11. Top: Abundance distribution for different $Y_{\mathrm{e}}$ (Wanajo et al. 2014). Bottom left: Expansion opacity as a function of wavelength for each $Y_{\mathrm{e}}$. Bottom right: Planck mean opacity as a function of temperature for each $Y_{\mathrm{e}}$.

Lippuner \& Roberts 2015; Kasen et al. 2015). Therefore, if the ejecta consists of material with $Y_{\mathrm{e}}>0.25$, a shortlived, bright and blue emission is expected due to the absence of high opacity lanthanide (Metzger \& Fernández 2014; Kasen et al. 2015; Tanaka et al. 2018; Wollaeger et al. 2018; Miller et al. 2019).

Thanks to the systematic atomic data, we are now able to connect the abundance pattern or electron fraction $Y_{\mathrm{e}}$ with the atomic opacities in a more reliable manner. For the mixture of the elements, we construct a line list from
Kurucz's line list (Kurucz \& Bell 1995) for $Z=1-28$, the VALD database (Piskunov et al. 1995; Ryabchikova et al. 1997; Kupka et al. 1999, 2000) for $Z=29$ and 30, and the results of our new atomic calculations for $Z=31-88$.

Figure 11 shows expansion opacities for different $Y_{\mathrm{e}}$. At $Y_{\mathrm{e}} \leq 0.20$, the opacities are dominated by lanthanide elements. The Planck opacities do not strongly depend on $Y_{\mathrm{e}}$ and stay around $\kappa \sim 20-30 \mathrm{~cm}^{2} \mathrm{~g}^{-1}$ at $T>5,000 \mathrm{~K}$. The temperature dependence at $T>5,000 \mathrm{~K}$ is weaker than in individual elements because of the mixture of the elements 
with different peaks positions as a function of temperature. Note that the opacities for the case of $Y_{\mathrm{e}}=0.10$ may be underestimated due to the lack of actinide elements in our calculations.

These low Ye cases $\left(Y_{\mathrm{e}}=0.10\right.$ and 0.15$)$ synthesize the heavy elements $(Z \geq 85)$ that show the large deviation of the energy levels compared with the NIST data. However, the impact to the opacities is limited. We calculate the opacities for the mixture of the elements by replacing the atomic data for $6 p$ and $7 s$ elements with those for $5 p$ and $6 s$ elements, respectively. We confirm that the Planck mean opacities are affected at most by $1 \%$ due to these changes. This is reasonable since the mass fractions of these heavy elements are small and the opacities of $s$-shell and $p$-shell elements are subdominant as compared with $f$-shell elements.

The opacities are smaller for higher $Y_{\mathrm{e}}$ as relative fractions of lanthanides decrease (Table 1). For $Y_{\mathrm{e}}=0.25-0.35$, the Planck mean opacities are dominated by the $d$-shell element (4th period in the periodic table) and they are in the range of $\kappa=1-10 \mathrm{~cm}^{2} \mathrm{~g}^{-1}$ at $T>5,000 \mathrm{~K}$. The opacities slightly increase with temperature due to the contribution of latter half of $d$-shell elements (group 8-11, see Figure 8). For $Y_{\mathrm{e}}=0.4$, the contributions from $d$-shell elements decrease and the opacities are even lower, i.e., $k=0.1-1 \mathrm{~cm}^{2} \mathrm{~g}^{-1}$ at $T>5,000 \mathrm{~K}$.

At a high temperature $(T \gtrsim 20,000 \mathrm{~K})$, the opacity of the low $Y_{\mathrm{e}}$ case decreases more rapidly than that of the high $Y_{\mathrm{e}}$ case. This is due to the limitation of ionization states in our atomic data (see Sections 3.1 and 3.2), i.e., our opacity data are not applicable for high temperature. Since the ionization potentials of $d$-shell elements are generally higher than those of $f$-shell elements, the applicable temperature range is wider for high $Y_{\mathrm{e}}$ cases, where $d$-shell elements dominate the opacities.

Note that the opacity of $\kappa=0.1-0.5 \mathrm{~cm}^{2} \mathrm{~g}^{-1}$ is often used for blue kilonovae because it gives a good approximation for Type Ia supernova, where $\mathrm{Fe}$ is the major component in the abundance. However, the opacities of mixture of $r$-process elements are almost always higher than $\kappa=0.1-0.5 \mathrm{~cm}^{2} \mathrm{~g}^{-1}$ even for high $Y_{\mathrm{e}}$, except for a low temperature $(T<2,000 \mathrm{~K})$. This is because Fe is not necessarily representative of $d$-shell elements and the contribution of Fe-like elements ( $\mathrm{Ru}$ and $\mathrm{Os}$ ) is low compared with other $d$-shell elements at $T<10,000 \mathrm{~K}$ (Figure 7 ).

For the ease of applications in analytical models, we give average values of the Planck mean opacities in Table 1. However, it is emphasized that the average opacities are derived only at $T=5,000-10,000 \mathrm{~K}$ and there is a strong temperature dependence at $T<5,000 \mathrm{~K}$.

\subsection{Time evolution of the opacity}

The opacities in the NS merger ejecta depend not only on elements and temperature but also the density of the ejecta (and thus, the position in the ejecta). Therefore, the opacities evolves with time by the combination of these effects. In this section, we apply our new atomic data to radiative transfer simulations of kilonovae and study the time variation of the opacities in the ejecta. We use a Monte-Carlo radiative transfer code developed by Tanaka \& Hotokezaka (2013); Tanaka et al. (2014) and further updated by Kawaguchi et al. (2018) to include special-
Table 1. Planck mean opacity for the mixture of the elements. $Y_{\mathrm{e}}$ is electron fraction, $X(\mathrm{La})$ is mass fraction of lanthanide elements, $X(\mathrm{La}+\mathrm{Ac})$ is mass fraction of lanthanide and actinide elements, and $\kappa$ is average Planck mean opacity for $T=5,000-10,000 \mathrm{~K}$ ( $\rho=1 \times 10^{-13} \mathrm{~g} \mathrm{~cm}^{-3}$ and $t=1$ day after the merger). The opacity shown with $*$ is underestimated due to the lack of complete atomic data for actinide elements.

\begin{tabular}{llll}
\hline$Y_{\mathrm{e}}$ & $X(\mathrm{La})$ & $X(\mathrm{La}+\mathrm{Ac})$ & $\begin{array}{l}\kappa \\
\mathrm{cm}^{2} \mathrm{~g}^{-1}\end{array}$ \\
\hline 0.10 & $7.1 \times 10^{-2}$ & $1.7 \times 10^{-1}$ & $19.5 *$ \\
0.15 & $2.6 \times 10^{-1}$ & $2.6 \times 10^{-1}$ & 32.2 \\
0.20 & $1.1 \times 10^{-1}$ & $1.1 \times 10^{-1}$ & 22.3 \\
0.25 & $5.5 \times 10^{-3}$ & $5.5 \times 10^{-3}$ & 5.60 \\
0.30 & $3.4 \times 10^{-7}$ & $3.4 \times 10^{-7}$ & 5.36 \\
0.35 & 0.0 & 0.0 & 3.30 \\
0.40 & 0.0 & 0.0 & 0.96 \\
& & & \\
$0.10-0.20$ & $2.1 \times 10^{-1}$ & $2.3 \times 10^{-1}$ & 30.7 \\
$0.20-0.30$ & $4.8 \times 10^{-2}$ & $4.8 \times 10^{-2}$ & 15.4 \\
$0.30-0.40$ & 0.0 & 0.0 & 4.68 \\
\hline
\end{tabular}

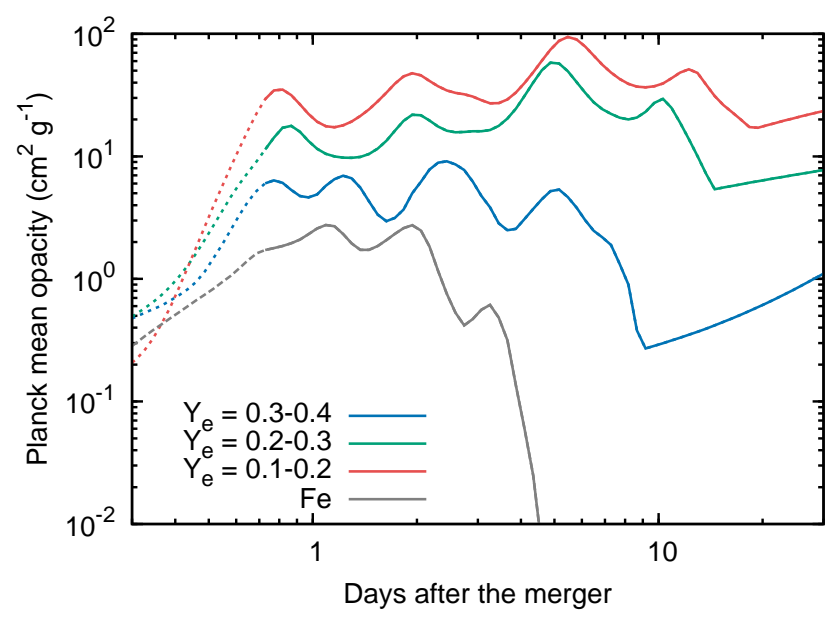

Figure 12. Time evolution of the Planck mean opacities at $v=0.1 c$ in the ejecta for the models with high $Y_{\mathrm{e}}\left(Y_{\mathrm{e}}=0.30-0.40\right.$, blue), intermediate $Y_{\mathrm{e}}\left(Y_{\mathrm{e}}=0.20-0.30\right.$, green $)$, and low $Y_{\mathrm{e}}\left(Y_{\mathrm{e}}=\right.$ $0.10-0.20$, red). The gray line shows the opacity of a model with pure Fe ejecta.

relativistic effects. We adopt a simple one-dimensional ejecta model with a power-law density structure $\rho \propto r^{-3}$ from $v=0.05 c$ to $v=0.2 c$ (Metzger et al. 2010; Metzger 2017), which gives an average velocity of $\langle v\rangle=0.1 c$. The total mass is set to be $M_{\mathrm{ej}}=0.03 M_{\odot}$.

The radiative transfer code adopts nuclear heating rates and abundances of $r$-process elements according to the value of $Y_{\mathrm{e}}$. Note that, for relatively high $Y_{\mathrm{e}}$, the nuclear heating rate strongly depends on $Y_{\mathrm{e}}$ since a few isotopes can dominate the heating rate in a certain timescale. Thus, an assumption of single $Y_{\mathrm{e}}$ ejecta, which is not the case in realistic conditions, can lead to misleading results. Therefore, we perform simulations with the following three $Y_{\mathrm{e}}$ ranges: high $Y_{\mathrm{e}}\left(Y_{\mathrm{e}}=0.30-0.40\right.$, no lanthanide), intermediate $Y_{\mathrm{e}}$ $\left(Y_{\mathrm{e}}=0.20-0.30\right.$, lanthanide fraction of $\left.\sim 5 \times 10^{-3}\right)$, and low $Y_{\mathrm{e}}\left(Y_{\mathrm{e}}=0.10-0.20\right.$, lanthanide fraction of $\left.\sim 0.1\right)$. The 


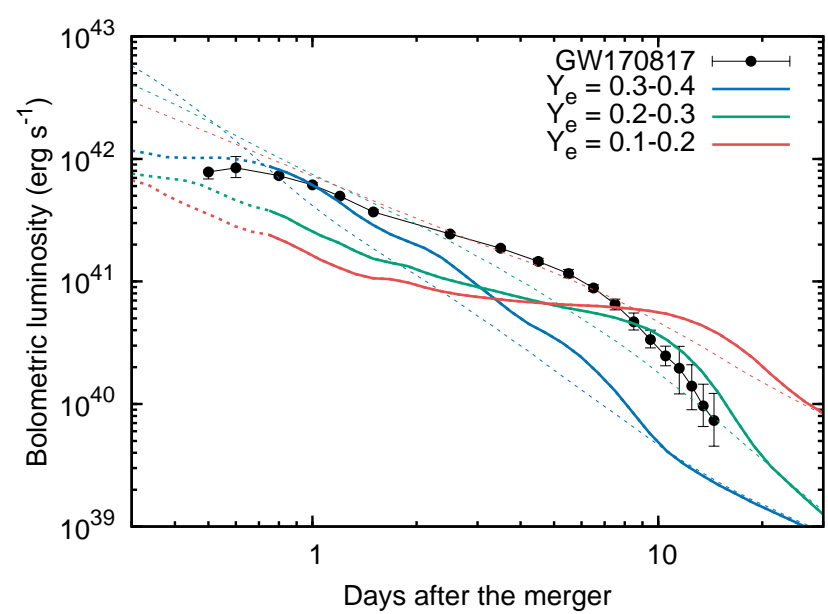

Figure 13. Bolometric light curves of the models with high $Y_{\mathrm{e}}$ $\left(Y_{\mathrm{e}}=0.30-0.40\right.$, blue $)$, intermediate $Y_{\mathrm{e}}\left(Y_{\mathrm{e}}=0.20-0.30\right.$, green $)$, and low $Y_{\mathrm{e}}\left(Y_{\mathrm{e}}=0.10-0.20\right.$, red $)$ compared with the bolometric light curve of GW170817/AT2017gfo constructed by Waxman et al. (2018). Dotted lines show the epoch in which our calculations are not reliable since the ejecta temperature is too high $(\mathrm{T}>20,000$ $\mathrm{K}$ ) for our opacity data (only up to triply ionized ions, see Section 3). Thin dashed lines show the luminosity deposited to the ejecta (radioactive power multiplied by thermalization efficiency) for each model.

heating rate and abundances are averaged over the $Y_{\mathrm{e}}$ range above by using single- $Y_{\mathrm{e}}$ nucleosynthesis calculations with a step of $\Delta Y_{\mathrm{e}}=0.01$ by Wanajo et al. (2014). The thermalization efficiencies of $\gamma$-rays, $\alpha$ particles, $\beta$ particles, and fission are separately taken into account by analytically estimating characteristic timescales (Barnes et al. 2016).

Figure 12 shows the time evolution of Planck mean opacity at $v=0.1 c$. It shows the total opacity but the boundbound opacity is always dominant. From $t=1$ to 10 days, the opacity increases with time for low and intermediate $Y_{\mathrm{e}}$ cases, while it slowly decreases for high $Y_{\mathrm{e}}$ case. The time evolution of the expansion opacity (Equation (2)) is controled by the competition between the term of $1 / \rho t$, which increases with time as $t^{2}$, and the summation of $1-e^{-\tau}$, which generally decreases with time as the lines get weaker for as the density decreases. The decrease of the line strength is more significant in the high $Y_{\mathrm{e}}$ case, which results in the temporal decrease of the opacity. Note that even with the increase in the opacity (for the low and intermediate $Y_{\mathrm{e}}$ cases), the optical depth of the ejecta, $\tau \sim \kappa \rho R$, decreases. In addition to the overall trend, the opacities shows temporal variation reflecting the temperature evolution, as shown in Figure 11. A significant decrease at $t \sim 10$ days corresponds to the sharp drop of the opacities at low temperature $T<2000 \mathrm{~K}$.

Overall degree of time variation is about an order of magnitude from $t=1$ to 10 days. This gives a caveat to the use of a constant opacity in the analysis of kilonova light curves, although it is useful to derive physical parameters. Figure 12 also shows a hypothetical case where the abundance of the high $Y_{\mathrm{e}}$ model is replaced with Fe. As discussed in Section 4.1, Fe is not a representative element for $d$-shell elements. Therefore, the use of Fe for high $Y_{\mathrm{e}}$ opaicty under-
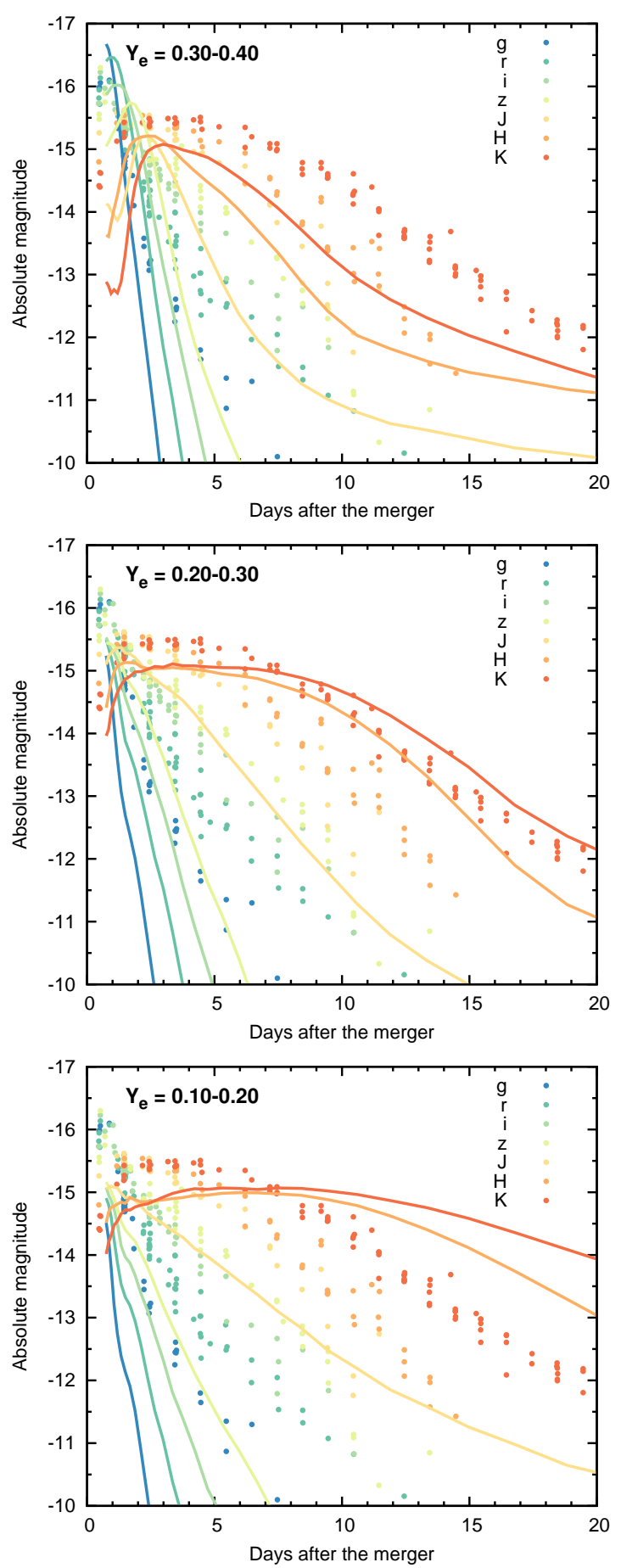

Figure 14. Multi-color light curves in optical (griz) and NIR $(J H K)$ filters for the models with high $Y_{\mathrm{e}}\left(Y_{\mathrm{e}}=0.30-0.40\right.$, top), intermediate $Y_{\mathrm{e}}\left(Y_{\mathrm{e}}=0.20-0.30\right.$, middle $)$, and low $Y_{\mathrm{e}}$ $\left(Y_{\mathrm{e}}=0.10-0.20\right.$, bottom $)$ compared with the observed light curves of GW170817/AT2017gfo (compiled by Villar et al. 2017).

estimates the opacity by a factor of $2-5$ up to $t=2-3$ days and by a factor of more than 10 at later time. 


\subsection{Light curves and spectra}

Finally we show the emergent light curves and spectra from the simulations in the previous section. Compared with our previous calculations (Tanaka et al. 2018) using only Se ( $p$ shell), $\mathrm{Ru}(d$-shell), Te ( $p$-shell), Nd $(f$-shell), and Er $(f$ shell) as representative elements, the light curves with new opacity data are more smooth both in time and wavelength. In particular, the use of representative elements can often exaggerate emission in certain wavelengths. At later time $(t \gtrsim 10$ days), only transitions from low-lying energy levels contribute the opacities. And thus, the use of small number of elements artificially enhances contributions from transitions of these elements. These effects are smeared out by properly including all the elements, which results in smooth spectra.

As expected from the properties of the opacities, the bolometric light curve of the model with high $Y_{\mathrm{e}}$ has a shorter timescale while that with low $Y_{\mathrm{e}}$ has a longer timescale (Figure 13). Compared with the observed luminosity of AT2017gfo associated with GW170817, the early $(t \sim 1-2$ days) light curve are most similar to the high $Y_{\mathrm{e}}$ model while the later light curve are most similar to the intermediate $Y_{\mathrm{e}}$ model.

These models also give a reasonable agreement with the multi-color light curves of AT2017gfo (Figure 14), although our models are very simple, and ejecta parameters such as mass and velocity are not tuned to reproduce the properties of AT2017gfo. The high $Y_{\mathrm{e}}$ model gives the early emission dominated in the optical wavelengths while the intermediate $Y_{\mathrm{e}}$ model gives the later emission dominated in the NIR wavelengths. It is emphasized that the optical/NIR flux ratio reflects the wavelength dependence of the opacities, and thus, cannot be accurately predicted by the calculations with a gray opacity.

The low $Y_{\mathrm{e}}$ model overproduces the total luminosity and gives too red color, which suggests that such a low $Y_{\mathrm{e}}$ component with a lanthanide fraction of $X(\mathrm{La}) \sim 0.1$ is not dominant $\left(M_{\mathrm{ej}} \ll 0.03 M_{\odot}\right)$. This is consistent with a relatively low lanthanide fraction $X(\mathrm{La})<0.01$ estimated by the spectral and light curve modelling (Chornock et al. 2017; Kasen et al. 2017; Kilpatrick et al. 2017; Tanaka et al. 2017; Tanvir et al. 2017).

The spectral features in our models are of interest because this is the first systematic calculations with the atomic data of the $r$-process elements. Figure 15 compares the model spectra with the observed spectra of GW170817/AT2017gfo with VLT/X-Shooter (Pian et al. 2017; Smartt et al. 2017). The models capture overall spectral shape and its evolution: the high $Y_{\mathrm{e}}$ model gives a similar shape of the optical spectra at early phases while intermediate $Y_{\mathrm{e}}$ model gives a similar NIR flux level at later phases.

However, detailed spectral features are not necessarily consistent between the observations and models. This is not surprising because our atomic data do not have an enough accuracy for each transition wavelength. To identify the spectral features, we need to use either wellcalibrated (but not complete) atomic data as done by Tanaka \& Hotokezaka (2013) and Watson et al. (2019) or very accurate atomic calculations as done by Gaigalas et al. (2019) and Radžiūtè et al. (2020).

There are two potentially important drawbacks in our models. One is too narrow spectral features in the early spectra. This is due to the assumption of $\langle v\rangle=0.1 c$ in our model. The observed broader features indicate that the lineforming region of the blue component should have $v>0.1 c$. In fact, such high velocities of the blue component have been suggested (e.g., Kilpatrick et al. 2017; McCully et al. 2017; Nicholl et al. 2017; Shappee et al. 2017). However, it was based on comparison with previous models, which could exaggerate the spectral features by the incompleteness in the atomic data. The comparison with our new model with the complete opacity data securely confirms the necessity of the high velocity for the blue component.

The other is the deficit of the optical flux at $t>5$ days after the merger. It is difficult to keep the optical flux at $t \gtrsim 5$ days because the optical flux in the high $Y_{\mathrm{e}}$ model declines too quickly and those in the intermediate and low $Y_{\mathrm{e}}$ models are suppressed too much. This difficulty remains even by changing ejecta mass and velocity. We may obtain a better agreement by assuming a lanthanide fraction somewhat lower than that in the intermediate $Y_{\mathrm{e}}$ model $\left(4.8 \times 10^{-2}\right)$. Such a relatively small lanthanide fraction is also supported by the modelling of the light curves (Chornock et al. 2017; Kilpatrick et al. 2017; Tanaka et al. 2017). Although such a intermediate lanthanide fraction requires a fine tuning of $Y_{\mathrm{e}}$, it can be naturally realized by the mixing in the ejecta as pointed by Metzger et al. (2018). Note that the interplay between multiple ejecta components also influences the light curve at this epoch (Kawaguchi et al. 2018, 2020). Alternatively, this difficulty might point out the necessity of more advanced radiative transfer calculations by taking into account non-LTE or fluorescence of numerous transitions, which are known to be important in supernovae (e.g., Baron et al. 1995; Pinto \& Eastman 2000; Mazzali 2000; Dessart \& Hillier 2005).

\section{SUMMARY}

We perform the first systematic atomic structure calculations for neutral atoms and singly, doubly, and triply ionized ions of the elements from $\mathrm{Fe}(Z=26)$ to $\operatorname{Ra}(Z=88)$ to understand the elemental variation of the bound-bound opacities in the NS merger ejecta. We find that the distributions of energy levels tend to be shifted to higher energy for increasing number of electrons in each shell. Also, the total number of excited levels is the highest for the half-closed, most complex elements. The combination of these two effects determines degree of contributions to the opacities. For typical temperature of kilonova $(T \sim 5,000 \mathrm{~K})$, elements with lower number of electrons have bigger contributions to the opacity thanks to the relatively low-lying energy levels. By this reason, Fe is not a good representative for the opacity of lanthanide-free ejecta. For a higher temperature $(T \gtrsim 10,000$ $\mathrm{K}$ ), elements with more electrons start to contribute because more transitions from excited levels become active.

The average opacities of mixture of $r$-process elements are $\kappa \sim 20-30 \mathrm{~cm}^{2} \mathrm{~g}^{-1}$ for $Y_{\mathrm{e}} \leq 0.20, \kappa \sim 3-5 \mathrm{~cm}^{2} \mathrm{~g}^{-1}$ for $Y_{\mathrm{e}}=0.25-0.35$, and $\kappa \sim 1 \mathrm{~cm}^{2} \mathrm{~g}^{-1}$ for $Y_{\mathrm{e}}=0.40$ at $T=5,000-10,000 \mathrm{~K}\left(\rho=1 \times 10^{-13} \mathrm{~g} \mathrm{~cm}^{-3}\right.$ and $t=1$ day $)$. Radiative transfer simulations with the new opacity data show that, even with the same abundance, the opacity in the ejecta changes with time. The opacity decreases with time 

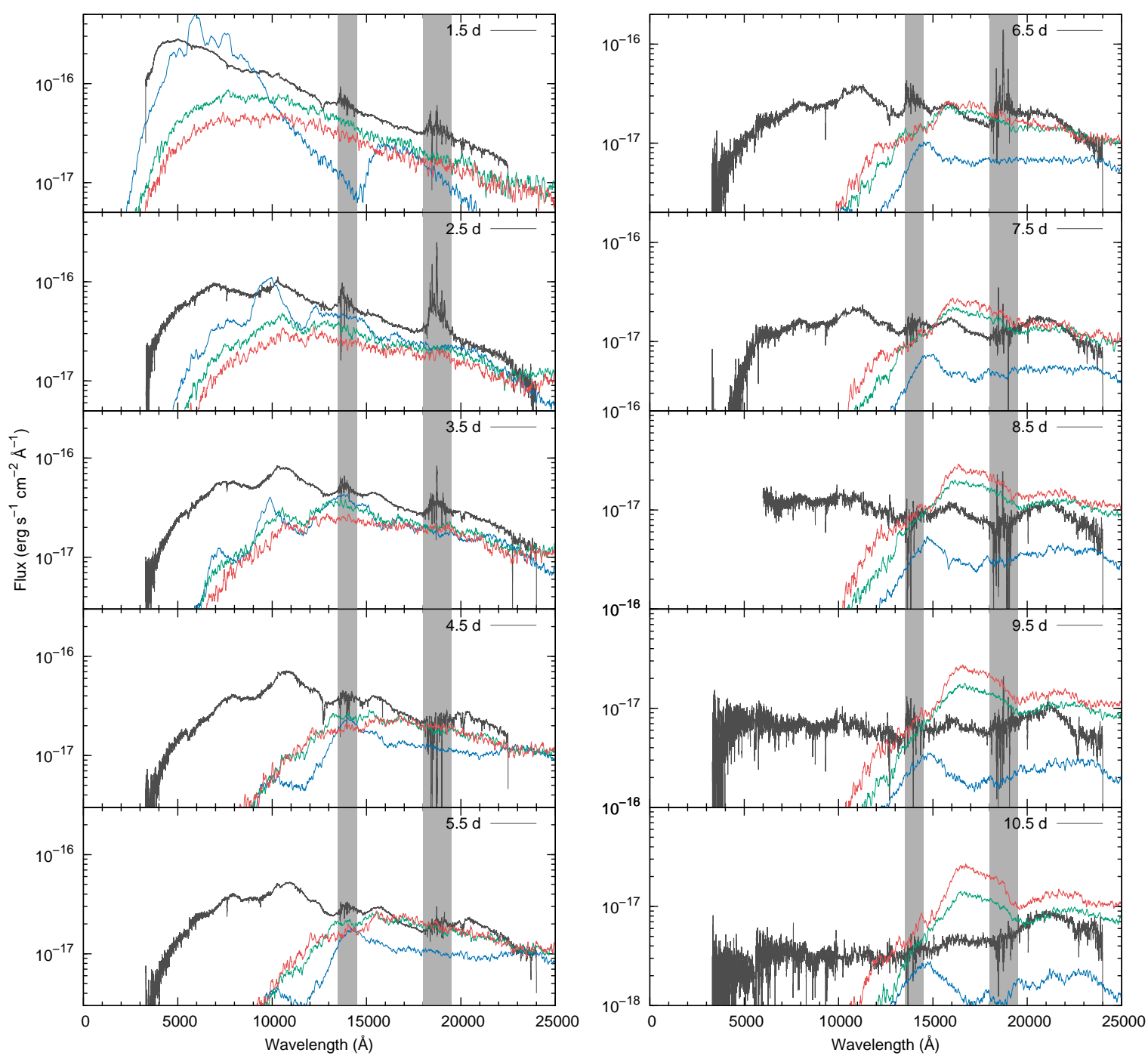

Figure 15. Spectral evolution of the models with high $Y_{\mathrm{e}}\left(Y_{\mathrm{e}}=0.30-0.40\right.$, blue $)$, intermediate $Y_{\mathrm{e}}\left(Y_{\mathrm{e}}=0.20-0.30\right.$, green $)$, and low $Y_{\mathrm{e}}$ $\left(Y_{\mathrm{e}}=0.10-0.20\right.$, red) compared with the spectra of GW170817/AT2017gfo taken with VLT/X-Shooter (Pian et al. 2017; Smartt et al. 2017, taken through the WISeREP, Yaron \& Gal-Yam 2012). The shaded areas show the wavelength ranges heavily affected by the atmospheric absorption.

for the model with high $Y_{\mathrm{e}}\left(Y_{\mathrm{e}}=0.30-0.40\right.$, no lanthanide), while it increases and then decreases for the models with intermediate $Y_{\mathrm{e}}\left(Y_{\mathrm{e}}=0.20-0.30\right.$, lanthanide fraction of $\sim$ $\left.5 \times 10^{-3}\right)$ and low $Y_{\mathrm{e}}\left(Y_{\mathrm{e}}=0.10-0.20\right.$, lanthanide fraction of $\sim 0.1)$. Overall variation is about an order of magnitude from $t=1$ to 10 days.

We confirm that multi-component ejecta are necessary to reproduce the observed properties of GW170817/AT2017gfo. The early blue part is best explained by the high $Y_{\mathrm{e}}$ model while the late NIR part is more similar to the model with intermediate $Y_{\mathrm{e}}$. The model with low $Y_{\mathrm{e}}$ overproduces the NIR light curves, which suggests that such a low $Y_{\mathrm{e}}$ component is not dominant $\left(M_{\mathrm{ej}} \ll 0.03 M_{\odot}\right)$.

Although our calculations provide opacities of a wide range of $r$-process elements, the detailed spectral features in the model cannot be compared with the observed spectra because our atomic data only focus on statistical properties and do not have enough accuracies in the transition wavelengths. To identify spectral features, combined use of accurate, well-calibrated (though not complete) atomic data will be important.

\section{ACKNOWLEDGEMENTS}

We thank Shinya Wanajo for providing the results of nucleosynthesis calculations, Michel Busquet for the generous support on the HULLAC code, and Brian Metzger for giving fruitful suggestions. MT and KK thank the Yukawa Institute for Theoretical Physics for support in the framework of International Molecule-type Workshop (YITP-T-18-06), where a part of this work has been done. Numerical simu- 
lations presented in this paper were carried out with Cray XC30 and XC50 at Center for Computational Astrophysics, National Astronomical Observatory of Japan.

This research was supported by JSPS Bilateral Joint Research Project, Inoue Science Research Award from Inoue Foundation for Science, the Grant-in-Aid for Scientific Research from JSPS (16H02183,19H00694,20H00158) and MEXT (17H06363), the NINS program of Promoting Research by Networking among Institutions (Grant Number 01411702). GG thanks the Research Council of Lithuania for funding his research (grant No. S-LJB-18-1).

\section{REFERENCES}

Abbott B. P., et al., 2017a, Physical Review Letters, 119, 161101 Abbott B. P., et al., 2017b, ApJ, 848, L12

Abbott B. P., et al., 2017c, ApJ, 848, L13

Andreoni I., et al., 2017, Publ. Astron. Soc. Australia, 34, e069

Arcavi I., et al., 2017, Nature, 551, 64

Bar-Shalom A., Klapisch M., Oreg J., 2001, J. Quant. Spectrosc. Radiative Transfer, 71, 169

Barnes J., Kasen D., Wu M.-R., Martínez-Pinedo G., 2016, ApJ, 829,110

Baron E., et al., 1995, ApJ, 441, 170

Chornock R., et al., 2017, ApJ, 848, L19

Coulter D. A., et al., 2017, Science, 358, 1556

Cowan R. D., 1981, The theory of atomic structure and spectra

Cowperthwaite P. S., et al., 2017, ApJ, 848, L17

Dessart L., Hillier D. J., 2005, A\&A, 437, 667

Díaz M. C., et al., 2017, ApJ, 848, L29

Drout M. R., et al., 2017, Science, 358, 1570

Eastman R. G., Pinto P. A., 1993, ApJ, 412, 731

Evans P. A., et al., 2017, Science, 358, 1565

Fernández R., Metzger B. D., 2013, MNRAS, 435, 502

Fernández R., Metzger B. $\quad$ D., 2016, Annual Review of Nuclear and Particle Science, 66, 23

Fernández R., Tchekhovskoy A., Quataert E., Foucart F., Kasen D., 2019, MNRAS, 482, 3373

Fontes C. J., Fryer C. L., Hungerford A. L., Wollaeger R. T., Rosswog S., Berger E., 2017, arXiv e-prints,

Fontes C. J., Fryer C. L., Hungerford A. L., Wollaeger R. T., Korobkin O., 2020, MNRAS, 493, 4143

Foucart F., O'Connor E., Roberts L., Kidder L. E., Pfeiffer H. P., Scheel M. A., 2016, Phys. Rev. D, 94, 123016

Fujibayashi S., Kiuchi K., Nishimura N., Sekiguchi Y., Shibata M., 2018, ApJ, 860, 64

Gaigalas G., Kato D., Rynkun P., Radžiūtè L., Tanaka M., 2019, ApJS, 240, 29

Goriely S., Bauswein A., Just O., Pllumbi E., Janka H.-T., 2015, MNRAS, 452, 3894

Hotokezaka K., Sari R., Piran T., 2017, MNRAS, 468, 91

Jönsson P., Gaigalas G., Bieroń J., Fischer C. F., Grant I. P., 2013, Computer Physics Communications, 184, 2197

Karp A. H., Lasher G., Chan K. L., Salpeter E. E., 1977, ApJ, 214,161

Kasen D., Thomas R. C., Nugent P., 2006, ApJ, 651, 366

Kasen D., Badnell N. R., Barnes J., 2013, ApJ, 774, 25

Kasen D., Fernández R., Metzger B. D., 2015, MNRAS, 450, 1777

Kasen D., Metzger B., Barnes J., Quataert E., Ramirez-Ruiz E., 2017, Nature, 551, 80

Kasliwal M. M., et al., 2017, Science, 358, 1559

Kawaguchi K., Shibata M., Tanaka M., 2018, ApJ, 865, L21

Kawaguchi K., Shibata M., Tanaka M., 2020, ApJ, 889, 171

Kilpatrick C. D., et al., 2017, Science, 358, 1583

Kramida A., Ralchenko Y., Reader J., NIST ASD Team 2018, NIST Atomic Spectra Database (version 5.6.1), https://physics.nist.gov/asd. National Institute of Standards and Technology, Gaithersburg, MD.

Kulkarni S. R., 2005, arXiv e-prints,

Kupka F., Piskunov N., Ryabchikova T. A., Stempels H. C., Weiss W. W., 1999, A\&AS, 138, 119

Kupka F. G., Ryabchikova T. A., Piskunov N. E., Stempels H. C., Weiss W. W., 2000, Baltic Astronomy, 9, 590

Kurucz R., Bell B., 1995, Atomic Line Data (R.L. Kurucz and B. Bell) Kurucz CD-ROM No. 23. Cambridge, Mass.: Smithsonian Astrophysical Observatory, 1995., 23

Li L.-X., Paczyński B., 1998, ApJ, 507, L59

Lippuner J., Roberts L. F., 2015, ApJ, 815, 82

Lipunov V. M., et al., 2017, ApJ, 850, L1

Mazzali P. A., 2000, A\&A, 363, 705

McCully C., et al., 2017, ApJ, 848, L32

Metzger B. D., 2017, Living Reviews in Relativity, 20, 3

Metzger B. D., Fernández R., 2014, MNRAS, 441, 3444

Metzger B. D., et al., 2010, MNRAS, 406, 2650

Metzger B. D., Thompson T. A., Quataert E., 2018, ApJ, 856, 101

Miller J. M., et al., 2019, Phys. Rev. D, 100, 023008

Nicholl M., et al., 2017, ApJ, 848, L18

Perego A., Radice D., Bernuzzi S., 2017, ApJ, 850, L37

Pian E., et al., 2017, Nature, 551, 67

Pinto P. A., Eastman R. G., 2000, ApJ, 530, 757

Piskunov N. E., Kupka F., Ryabchikova T. A., Weiss W. W., Jeffery C. S., 1995, A\&AS, 112, 525

Radice D., Galeazzi F., Lippuner J., Roberts L. F., Ott C. D., Rezzolla L., 2016, MNRAS, 460, 3255

Radice D., Perego A., Bernuzzi S., Zhang B., 2018a, MNRAS, 481, 3670

Radice D., Perego A., Hotokezaka K., Fromm S. A., Bernuzzi S., Roberts L. F., 2018b, ApJ, 869, 130

Radžiūtė L., Gaigalas G., Kato D., Rynkun P., Tanaka M., 2020, ApJS, 248, 17

Rosswog S., 2015, International Journal of Modern Physics D, 24,1530012

Rosswog S., Sollerman J., Feindt U., Goobar A., Korobkin O., Wollaeger R., Fremling C., Kasliwal M. M., 2018, A\&A, 615, A132

Ryabchikova T. A., Piskunov N. E., Kupka F., Weiss W. W., 1997, Baltic Astronomy, 6, 244

Sekiguchi Y., Kiuchi K., Kyutoku K., Shibata M., 2015, Phys. Rev. D, 91, 064059

Sekiguchi Y., Kiuchi K., Kyutoku K., Shibata M., Taniguchi K., 2016, Phys. Rev. D, 93, 124046

Shappee B. J., et al., 2017, Science, 358, 1574

Shibata M., Hotokezaka K., 2019, Annual Review of Nuclear and Particle Science, 69, 41

Shibata M., Fujibayashi S., Hotokezaka K., Kiuchi K., Kyutoku K., Sekiguchi Y., Tanaka M., 2017, Phys. Rev. D, 96, 123012

Siebert M. R., et al., 2017, ApJ, 848, L26

Smartt S. J., et al., 2017, Nature, 551, 75

Soares-Santos M., et al., 2017, ApJ, 848, L16

Sobolev V. V., 1960, Moving envelopes of stars

Tanaka M., 2016, Advances in Astronomy, 2016, 634197

Tanaka M., Hotokezaka K., 2013, ApJ, 775, 113

Tanaka M., Hotokezaka K., Kyutoku K., Wanajo S., Kiuchi K., Sekiguchi Y., Shibata M., 2014, ApJ, 780, 31

Tanaka M., et al., 2017, PASJ, 69, 102

Tanaka M., et al., 2018, ApJ, 852, 109

Tanvir N. R., et al., 2017, ApJ, 848, L27

Tominaga N., et al., 2018, PASJ, 70, 28

Troja E., et al., 2017, Nature, 551, 71

Utsumi Y., et al., 2017, PASJ, 69, 101

Valenti S., et al., 2017, ApJ, 848, L24

Villar V. A., et al., 2017, ApJ, 851, L21

Wanajo S., Sekiguchi Y., Nishimura N., Kiuchi K., Kyutoku K., Shibata M., 2014, ApJ, 789, L39 
Watson D., et al., 2019, Nature, 574, 497

Waxman E., Ofek E. O., Kushnir D., Gal-Yam A., 2018, MNRAS, 481, 3423

Wollaeger R. T., et al., 2018, MNRAS, 478, 3298

Yaron O., Gal-Yam A., 2012, PASP, 124, 668

\section{APPENDIX A: CONFIGURATIONS USED IN THE ATOMIC CALCULATIONS}

Table 2 summarizes our atomic calculations. For each ion, included configurations, the total number of levels, the total number of transitions, and the total number of transitions whose higher energy levels are below the ionization threshold (treated as bound-bound transitions in this paper) are given. All the configurations given in the table are taken into account in the RCI calculations. The configurations used for the energy minimization are shown in bold font.

In Figures A1 and A2, typical accuracy of our atomic calculations is shown for the selected elements: $\mathrm{Zr}$ II $(Z=$ $40, d)$, Nd II $(Z=60, f)$, and Hf II $(Z=72, f)$ which are important opacity source around $T=5,000 \mathrm{~K}$ and Mo III $(Z=42, d)$, Sm III $(Z=62, f)$, and W III $(Z=74, d)$ which are important around $T=10,000 \mathrm{~K}$. In the figures, the lowest energy level for each configuration is compared with that in the NIST ASD (Kramida et al. 2018). As also shown in Figure 2, a typical accuracy is about $<20 \%$ for singly ionized ions. The number of available data is smaller for more ionized ions (see top panel of Figure 2).

Impacts of the included configurations to the opacities are shown in the right panels of Figures A1 and A2. Different lines present the Planck mean opacities calculated with limited sets of configurations. For each ion, the atomic model is kept the same, and energy levels of certain configurations are removed to see the impact to the opacity. With our default choice of configuration (shown in filled blue circles in the left panels), the Planck mean opacities converge within $10 \%$. When more configurations are included, the number of transitions does increase. However, because these transitions have a large energy differences (short wavelengths) or they are from highly excited levels, they do not largely contribute to the overall opacities.

\section{APPENDIX B: VALIDITY OF THE SOBOLEV APPROXIMATION}

We discuss the validity of the Sobolev approximation, which we use to evaluate the bound-bound opacities in the NS merger ejecta. When the wavelength spacing of the lines $\left(\Delta \lambda_{\text {line }}\right)$ becomes as small as the thermal width of the lines $\left(\Delta \lambda_{\mathrm{th}}\right)$, overlap of the lines becomes severe and the Sobolev approximation is not applicable (Kasen et al. 2013). In a typical condition of the ejecta at $t=1$ day, the thermal velocity is $v_{\text {th }} \simeq 0.7 \mathrm{~km} \mathrm{~s}^{-1}(A / 150)^{-1 / 2}(T / 5,000 \mathrm{~K})^{1 / 2}$, where $A$ is the mass number. Therefore, the thermal width of the line is $\Delta \lambda_{\text {th }}=\left(v_{\text {th }} / c\right) \lambda \simeq 0.01 \AA(A / 150)^{-1 / 2}(T / 5,000 \mathrm{~K})^{1 / 2}(\lambda / 5,000$ $\AA)$. We can calculate typical line spacing by $\Delta \lambda_{\text {line }}=\Delta \lambda / N$, where $\Delta \lambda$ is the wavelength bin in the calculation and $N$ is the number of the strong lines in the bin. Figure A3 shows the ratio $\Delta \lambda_{\text {th }} / \Delta \lambda_{\text {line }}$ for the ejecta with pure lanthanide composition (top and middle panels) and for the ejecta with the mixture of the elements (bottom panels). The line spacing is evaluated by counting the number of lines with $\tau_{l}>1$. For all the cases, the ratio $\Delta \lambda_{\text {th }} / \Delta \lambda_{\text {line }}$ is smaller than unity, i.e., $\Delta \lambda_{\text {line }}>\Delta \lambda_{\text {th }}$, at the relevant wavelength range. This relation holds even if the line spacing is evaluated by including weaker lines with $\tau>0.1$. Therefore, the use of Sobolev optical depth is a sound approximation even in the lanthaniderich ejecta of NS merger.

This paper has been typeset from a $\mathrm{TE}_{\mathrm{E}} \mathrm{X} / \mathrm{LATEX}$ file prepared by the author. 
Table B1. Summary of HULLAC calculations. The last column shows the number of transitions whose upper level is below the ionization potential.

\begin{tabular}{|c|c|c|c|c|}
\hline Ion & Configurations & $N_{\text {level }}$ & $N_{\text {line }}$ & $N_{\text {line }}^{*}$ \\
\hline Fe I & $\begin{array}{l}3 \mathbf{d}^{6} \mathbf{4} \mathbf{s}^{2}, \mathbf{3} \mathbf{d}^{8}, 3 d^{7} 4 s^{1}, 3 d^{6} 4 s^{1} 4 p^{1}, 3 d^{7} 4 p^{1} \\
3 d^{5} 4 s^{2} 4 p^{1}, 3 d^{6} 4 s^{1} 5 s^{1}, 3 d^{7} 5 s^{1}, 3 d^{6} 4 s^{1} 5 p^{1}, 3 d^{6} 4 s^{1} 4 d^{1} \\
3 d^{7} 5 p^{1}, 3 d^{7} 4 d^{1}, 3 d^{6} 4 s^{1} 6 s^{1}, 3 d^{7} 6 s^{1}, 3 d^{6} 4 s^{1} 6 p^{1} \\
3 d^{6} 4 s^{1} 5 d^{1}\end{array}$ & 3195 & 1130295 & 26490 \\
\hline Fe II & $\begin{array}{l}3 \mathbf{d}^{6} \mathbf{4} \mathbf{s}^{1}, \mathbf{3 \mathbf { d } ^ { 7 }}, 3 d^{5} 4 s^{2}, 3 d^{6} 4 p^{1}, 3 d^{5} 4 s^{1} 4 p^{1} \\
3 d^{6} 5 s^{1}, 3 d^{6} 4 d^{1}, 3 d^{6} 5 p^{1}, 3 d^{6} 6 s^{1}, 3 d^{6} 4 f^{1} \\
3 d^{5} 4 s^{1} 5 s^{1}, 3 d^{6} 5 d^{1}, 3 d^{6} 6 p^{1}, 3 d^{5} 4 p^{2}, 3 d^{5} 4 s^{1} 4 d^{1} \\
3 d^{6} 7 s^{1}\end{array}$ & 3467 & 1253693 & 73267 \\
\hline Fe III & $\begin{array}{l}3 d^{6}, 3 d^{5} 4 s^{1}, 3 d^{5} 4 p^{1}, 3 d^{5} 4 d^{1}, 3 d^{5} 5 s^{1} \\
3 d^{5} 5 p^{1}, 3 d^{5} 4 f^{1}, 3 d^{4} 4 s^{1} 4 p^{1}, 3 d^{5} 5 d^{1}, 3 d^{5} 6 s^{1} \\
3 d^{5} 6 p^{1}\end{array}$ & 2338 & 560985 & 120376 \\
\hline Fe IV & $\begin{array}{l}3 \mathbf{d}^{5}, 3 d^{4} 4 s^{1}, 3 d^{4} 4 p^{1}, 3 d^{3} 4 s^{1} 4 p^{1}, 3 d^{4} 5 s^{1} \\
3 d^{4} 5 p^{1}\end{array}$ & 736 & 45182 & 38785 \\
\hline Co I & $\begin{array}{l}\mathbf{3} \mathbf{p}^{\mathbf{6}} \mathbf{3} \mathbf{d}^{\mathbf{7}} \mathbf{4} \mathbf{s}^{\mathbf{2}}, \mathbf{3} \mathbf{p}^{\mathbf{6}} \mathbf{3} \mathbf{d}^{\mathbf{8}} \mathbf{4} \mathbf{s}^{1}, \mathbf{3 p}^{\mathbf{6}} \mathbf{3} \mathbf{d}^{\mathbf{9}}, 3 p^{6} 3 d^{7} 4 s^{1} 4 p^{1}, 3 p^{6} 3 d^{8} 4 p^{1}, \\
3 p^{6} 3 d^{8} 5 s^{1}, 3 p^{6} 3 d^{7} 4 s^{1} 5 s^{1}, 3 p^{6} 3 d^{8} 4 d^{1}, 3 p^{6} 3 d^{7} 4 s^{1} 4 d^{1}\end{array}$ & 778 & 64798 & 7619 \\
\hline Co II & $\begin{array}{l}3 \mathbf{d}^{8}, 3 d^{7} 4 s^{1}, 3 d^{6} 4 s^{2}, 3 d^{7} 4 p^{1}, 3 d^{6} 4 s^{1} 4 p^{1} \\
3 d^{7} 5 s^{1}, 3 d^{7} 4 d^{1}, 3 d^{7} 5 p^{1}, 3 d^{7} 6 s^{1}\end{array}$ & 905 & 87188 & 13324 \\
\hline Co III & $3 \mathbf{d}^{7}, 3 d^{6} 4 s^{1}, 3 d^{6} 4 p^{1}, 3 d^{6} 4 d^{1}, 3 d^{6} 5 s^{1}$ & 601 & 35051 & 32983 \\
\hline Co IV & 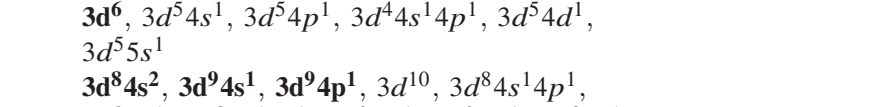 & 1088 & 130730 & 57610 \\
\hline $\mathrm{Ni} \mathrm{I}$ & $\begin{array}{l}3 d^{9} 5 s^{1}, 3 d^{8} 4 s^{1} 5 s^{1}, 3 d^{9} 5 p^{1}, 3 d^{9} 4 d^{1}, 3 d^{9} 6 s^{1} \\
3 d^{9} 6 p^{1}, 3 d^{9} 5 d^{1}, 3 d^{9} 4 f^{1} \\
\mathbf{3 p}^{6} \mathbf{3}^{9}, 3 p^{6} 3 d^{8} 4 s^{1}, 3 p^{6} 3 d^{7} 4 s^{2}, 3 p^{6} 3 d^{8} 4 p^{1}, 3 p^{6} 3 d^{7} 4 s^{1} 4 p^{1}\end{array}$ & 236 & 7042 & 1233 \\
\hline Ni II & $\begin{array}{l}3 p^{6} 3 d^{8} 5 s^{1}, 3 p^{6} 3 d^{8} 4 d^{1}, 3 p^{6} 3 d^{8} 5 p^{1}, 3 p^{6} 3 d^{8} 6 s^{1}, 3 p^{6} 3 d^{8} 4 f^{1}, \\
3 p^{6} 3 d^{8} 5 d^{1}\end{array}$ & 587 & 38893 & 8360 \\
\hline Ni III & $\begin{array}{l}3 \mathbf{p}^{6} \mathbf{3} \mathbf{d}^{8}, 3 p^{6} 3 d^{7} 4 s^{1}, 3 p^{6} 3 d^{7} 4 p^{1}, 3 p^{6} 3 d^{6} 4 s^{2}, 3 p^{6} 3 d^{7} 4 d^{1} \\
3 p^{6} 3 d^{7} 5 s^{1}, 3 p^{6} 3 d^{7} 5 p^{1}, 3 p^{6} 3 d^{6} 4 s^{1} 4 p^{1}\end{array}$ & 867 & 76483 & 28070 \\
\hline Ni IV & $\begin{array}{l}3 \mathbf{p}^{6} 3 \mathbf{d}^{7}, 3 p^{6} 3 d^{6} 4 s^{1}, 3 p^{6} 3 d^{6} 4 p^{1}, 3 p^{6} 3 d^{5} 4 s^{2}, 3 p^{6} 3 d^{6} 4 d^{1} \\
3 p^{6} 3 d^{6} 5 s^{1}, 3 p^{6} 3 d^{6} 5 p^{1}\end{array}$ & 818 & 76592 & 75409 \\
\hline $\mathrm{Cu} \mathrm{I}$ & 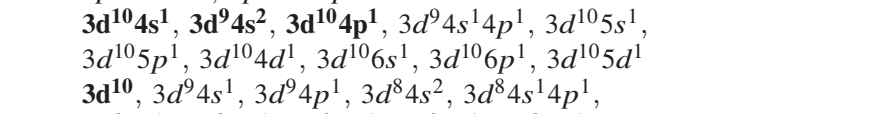 & 38 & 186 & 186 \\
\hline $\mathrm{Cu} \mathrm{II}$ & $\begin{array}{l}3 d^{9} 5 s^{1}, 3 d^{9} 4 d^{1}, 3 d^{9} 5 p^{1}, 3 d^{9} 6 s^{1}, 3 d^{9} 4 f^{1}, \\
3 d^{9} 5 d^{1}, 3 d^{9} 6 p^{1} \\
\mathbf{3 p}^{6} 3 \mathbf{d}^{9}, 3 p^{6} 3 d^{8} 4 s^{1}, 3 p^{6} 3 d^{8} 4 p^{1}, 3 p^{6} 3 d^{7} 4 s^{2}, 3 p^{6} 3 d^{8} 5 s^{1},\end{array}$ & 204 & 4559 & 390 \\
\hline $\mathrm{Cu}$ III & $\begin{array}{l}3 p^{6} 3 d^{8} 4 d^{1}, 3 p^{6} 3 d^{8} 5 p^{1}, 3 p^{6} 3 d^{7} 4 s^{1} 4 p^{1}, 3 p^{6} 3 d^{8} 4 f^{1}, 3 p^{6} 3 d^{8} 6 s^{1}, \\
3 p^{6} 3 d^{8} 5 d^{1}\end{array}$ & 587 & 38893 & 17666 \\
\hline $\mathrm{Cu}$ IV & $\begin{array}{l}\mathbf{3 p}^{6} 3 \mathbf{d}^{8}, 3 p^{6} 3 d^{7} 4 s^{1}, 3 p^{6} 3 d^{7} 4 p^{1}, 3 p^{6} 3 d^{7} 4 d^{1}, 3 p^{6} 3 d^{6} 4 s^{2}, \\
3 p^{6} 3 d^{7} 5 s^{1}\end{array}$ & 397 & 14803 & 14595 \\
\hline Zn I & $\begin{array}{l}\mathbf{3 d}^{10} 4 \mathbf{s}^{2}, 3 d^{10} 4 s^{1} 4 p^{1}, 3 d^{10} 4 s^{1} 5 s^{1}, 3 d^{10} 4 s^{1} 5 p^{1}, 3 d^{10} 4 s^{1} 4 d^{1} \\
3 d^{10} 4 s^{1} 6 s^{1}, 3 d^{10} 4 s^{1} 6 p^{1}, 3 d^{10} 4 s^{1} 5 d^{1}, 3 d^{10} 4 s^{1} 4 f^{1} \\
\mathbf{3 d}^{\mathbf{1 0}} 4 \mathbf{s}^{1}, 3 d^{10} 4 p^{1}, 3 d^{9} 4 s^{2}, 3 d^{10} 5 s^{1}, 3 d^{10} 4 d^{1}\end{array}$ & 29 & 130 & 130 \\
\hline Zn II & $\begin{array}{l}3 d^{10} 5 p^{1}, 3 d^{9} 4 s^{1} 4 p^{1}, 3 d^{10} 6 s^{1}, 3 d^{10} 4 f^{1}, 3 d^{10} 5 d^{1} \\
3 d^{10} 6 p^{1}\end{array}$ & 40 & 195 & 156 \\
\hline Zn III & $\begin{array}{l}3 \mathbf{d}^{10}, 3 d^{9} 4 s^{1}, 3 d^{9} 4 p^{1}, 3 d^{8} 4 s^{2}, 3 d^{9} 4 d^{1} \\
3 d^{9} 5 s^{1}, 3 d^{9} 5 p^{1}, 3 d^{8} 4 s^{1} 4 p^{1}\end{array}$ & 150 & 2206 & 510 \\
\hline Zn IV & $\begin{array}{l}3 \mathbf{p}^{6} \mathbf{3} \mathbf{d}^{9}, 3 p^{6} 3 d^{8} 4 s^{1}, 3 p^{6} 3 d^{8} 4 p^{1}, 3 p^{6} 3 d^{8} 4 d^{1}, 3 p^{6} 3 d^{8} 5 p^{1} \\
3 p^{6} 3 d^{8} 4 f^{1}, 3 p^{6} 3 d^{8} 6 p^{1}, 3 p^{6} 3 d^{8} 5 f^{1}\end{array}$ & 382 & 12353 & 12168 \\
\hline Ga I & $\begin{array}{l}4 \mathbf{s}^{2} \mathbf{4} \mathbf{p}^{1}, 4 s^{2} 5 s^{1}, 4 s^{2} 5 p^{1}, 4 s^{2} 4 d^{1}, 4 s^{2} 6 s^{1} \\
4 s^{1} 4 p^{2}, 4 s^{2} 6 p^{1}, 4 s^{2} 5 d^{1}, 4 s^{2} 4 f^{1}\end{array}$ & 22 & 85 & 36 \\
\hline Ga II & $\begin{array}{l}4 s^{2}, 4 s^{1} 4 p^{1}, 4 s^{1} 5 s^{1}, 4 p^{2}, 4 s^{1} 4 d^{1} \\
4 s^{1} 5 p^{1}, 4 s^{1} 6 s^{1}, 4 s^{1} 5 d^{1}\end{array}$ & 26 & 100 & 100 \\
\hline Ga III & $\begin{array}{l}3 \mathbf{d}^{10} \mathbf{4} \mathbf{s}^{1}, 3 d^{10} 4 p^{1}, 3 d^{9} 4 s^{2}, 3 d^{10} 5 s^{1}, 3 d^{10} 4 d^{1} \\
3 d^{10} 5 p^{1}, 3 d^{10} 4 f^{1}\end{array}$ & 12 & 26 & 26 \\
\hline Ga IV & $\begin{array}{l}\mathbf{3 d ^ { 1 0 }}, 3 d^{9} 4 s^{1}, 3 d^{9} 4 p^{1}, 3 d^{9} 4 d^{1}, 3 d^{9} 5 s^{1} \\
3 d^{9} 5 p^{1}\end{array}$ & 51 & 396 & 396 \\
\hline
\end{tabular}



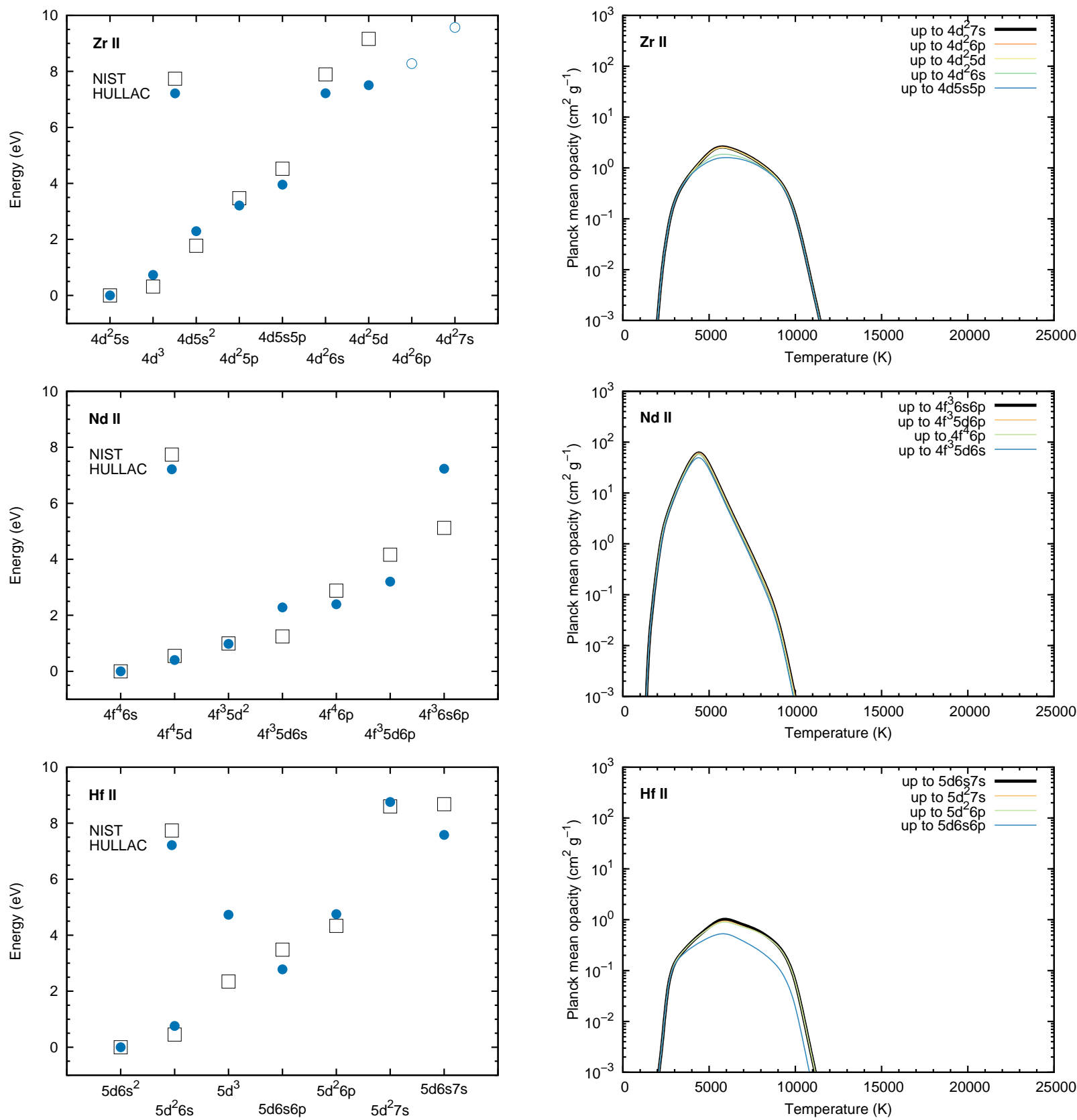

Figure A1. Left: Lowest energy level of each configuration for Zr II $(Z=40, d)$, Nd II $(Z=60, f)$, and Hf II $(Z=72, f)$. Blue circles show our calculations while black squares show the data in the NIST ASD (Kramida et al. 2018). For our calculations, filled circles represent the configurations included in our default set and open circles represent the configurations added for the convergence studies of the opacities. Right: Planck mean opacities as a function of temperature, which are calculated with limited sets of configurations. Our default calculations include the configurations up to $4 d^{2} 5 d$ (Zr II), $4 f^{3} 6 s 6 p$ (Nd II), and $5 d 6 s 7 s$ (Hf II). 

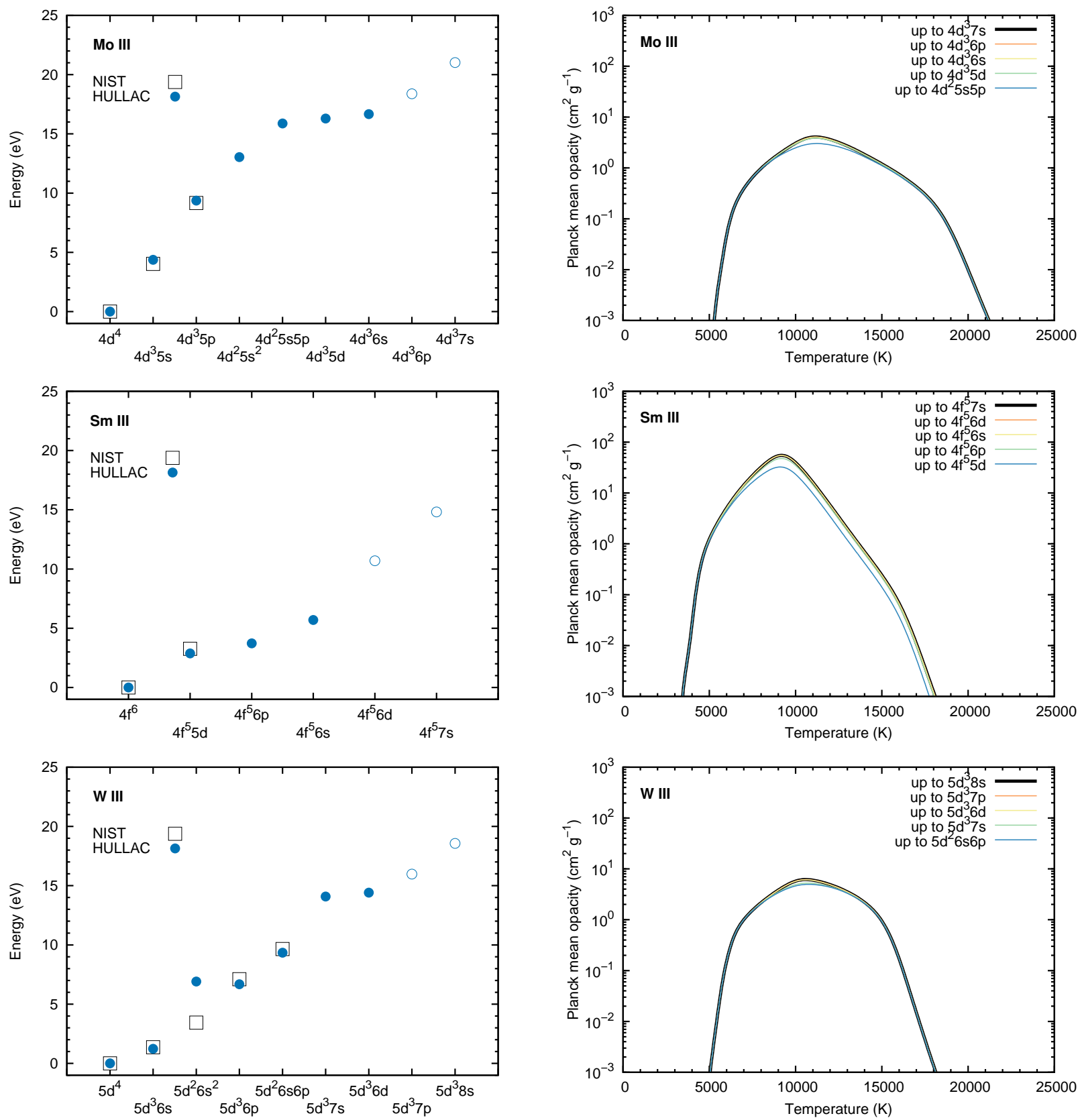

Figure A2. Same as Figure A1 but for Mo III $(Z=42, d)$, Sm III $(Z=62, f)$, and W III $(Z=74, d)$. Our default calculations include the configurations up to $4 d^{3} 6 s$ (Mo III), $4 f^{5} 6 s$ (Sm III), and $5 d^{3} 6 d$ (W III). 
Table B1 - continued Summary of HULLAC calculations. The last column shows the number of transitions whose upper level is below the ionization potential.

\begin{tabular}{|c|c|c|c|c|}
\hline Ion & Configurations & $N_{\text {level }}$ & $N_{\text {line }}$ & $N_{\text {line }}^{*}$ \\
\hline Ge I & 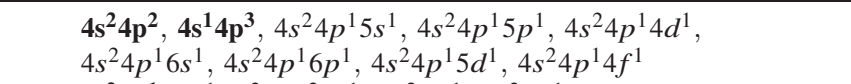 & 79 & 966 & 106 \\
\hline Ge II & $\begin{array}{l}4 \mathbf{s}^{2} \mathbf{4} \mathbf{p}^{1}, 4 s^{1} 4 p^{2}, 4 s^{2} 5 s^{1}, 4 s^{2} 5 p^{1}, 4 s^{2} 4 d^{1} \\
4 s^{2} 6 s^{1}, 4 s^{2} 5 d^{1}, 4 s^{2} 4 f^{1}, 4 s^{2} 6 p^{1}\end{array}$ & 22 & 85 & 85 \\
\hline Ge III & $\begin{array}{l}3 \mathbf{d}^{10} 4 s^{2}, 3 d^{10} 4 s^{1} 4 p^{1}, 3 d^{10} 4 p^{2}, 3 d^{10} 4 s^{1} 5 s^{1}, 3 d^{10} 4 s^{1} 4 d^{1} \\
3 d^{10} 4 s^{1} 5 p^{1}, 3 d^{10} 4 s^{1} 4 f^{1}, 3 d^{10} 4 s^{1} 6 s^{1}, 3 d^{10} 4 s^{1} 5 d^{1}, 3 d^{10} 4 s^{1} 7 s^{1}\end{array}$ & 32 & 144 & 144 \\
\hline Ge IV & $\begin{array}{l}3 \mathbf{d}^{10} 4 \mathbf{s}^{1}, 3 d^{10} 4 p^{1}, 3 d^{10} 4 d^{1}, 3 d^{10} 5 s^{1}, 3 d^{10} 5 p^{1} \\
3 d^{10} 4 f^{1}, 3 d^{9} 4 s^{2}, 3 d^{10} 5 d^{1}, 3 d^{10} 6 s^{1}\end{array}$ & 15 & 39 & 39 \\
\hline As I & $\begin{array}{l}4 s^{2} 4 \mathbf{p}^{3}, 4 s^{2} 4 p^{2} 5 s^{1}, 4 s^{1} 4 p^{4}, 4 s^{2} 4 p^{2} 5 p^{1}, 4 s^{2} 4 p^{2} 4 d^{1} \\
4 s^{2} 4 p^{2} 6 s^{1}, 4 s^{2} 4 p^{2} 6 p^{1}\end{array}$ & 99 & 1738 & 1096 \\
\hline As II & $\begin{array}{l}4 s^{2} 4 \mathbf{p}^{2}, 4 s^{1} 4 p^{3}, 4 s^{2} 4 p^{1} 5 s^{1}, 4 s^{2} 4 p^{1} 4 d^{1}, 4 s^{2} 4 p^{1} 5 p^{1} \\
4 s^{2} 4 p^{1} 6 s^{1}, 4 s^{2} 4 p^{1} 5 d^{1}, 4 s^{2} 4 p^{1} 6 p^{1}\end{array}$ & 67 & 707 & 684 \\
\hline As III & $\begin{array}{l}4 \mathbf{s}^{2} \mathbf{4} \mathbf{p}^{1}, 4 s^{1} 4 p^{2}, 4 s^{2} 5 s^{1}, 4 s^{2} 4 d^{1}, 4 s^{2} 5 p^{1} \\
4 p^{3}, 4 s^{2} 6 s^{1}, 4 s^{2} 4 f^{1}, 4 s^{2} 5 d^{1}\end{array}$ & 25 & 122 & 122 \\
\hline As IV & $\begin{array}{l}3 \mathbf{d}^{10} 4 \mathbf{s}^{2}, 3 d^{10} 4 s^{1} 4 p^{1}, 3 d^{10} 4 s^{1} 4 d^{1}, 3 d^{10} 4 p^{2}, 3 d^{10} 4 s^{1} 5 s^{1} \\
3 d^{10} 4 s^{1} 5 p^{1}, 3 d^{10} 4 s^{1} 4 f^{1}, 3 d^{10} 4 s^{1} 5 d^{1}, 3 d^{10} 4 s^{1} 6 s^{1}\end{array}$ & 30 & 131 & 131 \\
\hline Se I & $\begin{array}{l}\mathbf{4} \mathbf{s}^{2} 4 \mathbf{p}^{4}, 4 s^{2} 4 p^{3} 5 s^{1}, 4 s^{2} 4 p^{3} 5 p^{1}, 4 s^{2} 4 p^{3} 4 d^{1}, 4 s^{2} 4 p^{3} 6 s^{1} \\
4 s^{2} 4 p^{3} 6 p^{1}, 4 s^{2} 4 p^{3} 5 d^{1}\end{array}$ & 157 & 3600 & 971 \\
\hline Se II & $\begin{array}{l}\mathbf{4} \mathbf{s}^{2} 4 \mathbf{p}^{3}, 4 s^{1} 4 p^{4}, 4 s^{2} 4 p^{2} 5 s^{1}, 4 s^{2} 4 p^{2} 5 p^{1}, 4 s^{2} 4 p^{2} 6 s^{1} \\
4 s^{2} 4 p^{2} 5 d^{1}\end{array}$ & 78 & 970 & 927 \\
\hline Se III & $\begin{array}{l}4 \mathbf{s}^{2} 4 \mathbf{p}^{2}, 4 s^{1} 4 p^{3}, 4 s^{2} 4 p^{1} 4 d^{1}, 4 s^{2} 4 p^{1} 5 s^{1}, 4 s^{2} 4 p^{1} 5 p^{1} \\
4 s^{2} 4 p^{1} 6 s^{1}, 4 s^{2} 4 p^{1} 5 d^{1}\end{array}$ & 57 & 419 & 419 \\
\hline Se IV & $\begin{array}{l}4 \mathbf{s}^{\mathbf{2}} \mathbf{4} \mathbf{p}^{\mathbf{1}}, 4 s^{1} 4 p^{2}, 4 s^{2} 4 d^{1}, 4 s^{2} 5 s^{1}, 4 p^{3} \\
4 s^{2} 5 p^{1}\end{array}$ & 20 & 86 & 86 \\
\hline $\mathrm{Br} \mathrm{I}$ & $\begin{array}{l}4 \mathbf{s}^{2} 4 \mathbf{p}^{5}, 4 s^{2} 4 p^{4} 5 s^{1}, 4 s^{2} 4 p^{4} 5 p^{1}, 4 s^{2} 4 p^{4} 4 d^{1}, 4 s^{2} 4 p^{4} 6 s^{1} \\
4 s^{1} 4 p^{6}, 4 s^{2} 4 p^{4} 6 p^{1}, 4 s^{2} 4 p^{4} 5 d^{1}\end{array}$ & 117 & 2166 & 1102 \\
\hline Br II & $\begin{array}{l}4 s^{2} 4 \mathbf{p}^{4}, 4 s^{2} 4 p^{3} 5 s^{1}, 4 s^{1} 4 p^{5}, 4 s^{2} 4 p^{3} 4 d^{1}, 4 s^{2} 4 p^{3} 5 p^{1} \\
4 s^{2} 4 p^{3} 6 s^{1}, 4 s^{2} 4 p^{3} 5 d^{1}, 4 s^{2} 4 p^{3} 4 f^{1}\end{array}$ & 173 & 4023 & 3490 \\
\hline Br III & $\begin{array}{l}4 \mathbf{s}^{2} 4 \mathbf{p}^{3}, 4 s^{1} 4 p^{4}, 4 s^{2} 4 p^{2} 4 d^{1}, 4 s^{2} 4 p^{2} 5 s^{1}, 4 s^{2} 4 p^{2} 5 p^{1} \\
4 s^{2} 4 p^{2} 5 d^{1}, 4 s^{2} 4 p^{2} 6 s^{1}\end{array}$ & 106 & 1448 & 1448 \\
\hline $\mathrm{Br}$ IV & $\begin{array}{l}4 \mathbf{s}^{2} 4 \mathbf{p}^{2}, 4 s^{1} 4 p^{3}, 4 s^{2} 4 p^{1} 4 d^{1}, 4 s^{2} 4 p^{1} 5 s^{1}, 4 s^{2} 4 p^{1} 5 p^{1} \\
4 s^{2} 4 p^{1} 6 s^{1}\end{array}$ & 45 & 309 & 309 \\
\hline Kr I & $\begin{array}{l}4 \mathbf{s}^{\mathbf{2}} \mathbf{4 \mathbf { p } ^ { 6 }}, 4 s^{2} 4 p^{5} 5 s^{1}, 4 s^{2} 4 p^{5} 5 p^{1}, 4 s^{2} 4 p^{5} 4 d^{1}, 4 s^{2} 4 p^{5} 6 s^{1}, \\
4 s^{2} 4 p^{5} 6 p^{1}, 4 s^{2} 4 p^{5} 5 d^{1}\end{array}$ & 53 & 434 & 434 \\
\hline Kr II & 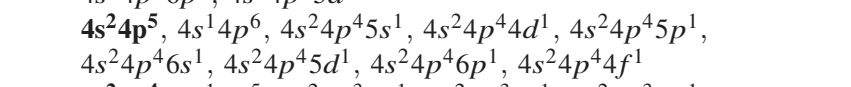 & 147 & 3305 & 2903 \\
\hline Kr III & $\begin{array}{l}4 \mathbf{s}^{2} 4 \mathbf{p}^{4}, 4 s^{1} 4 p^{5}, 4 s^{2} 4 p^{3} 4 d^{1}, 4 s^{2} 4 p^{3} 5 s^{1}, 4 s^{2} 4 p^{3} 5 p^{1} \\
4 s^{2} 4 p^{3} 6 s^{1}, 4 s^{2} 4 p^{3} 5 d^{1}, 4 s^{2} 4 p^{3} 6 d^{1}, 4 p^{6}\end{array}$ & 172 & 2786 & 2749 \\
\hline Kr IV & $\begin{array}{l}4 s^{2} 4 \mathbf{p}^{3}, 4 s^{1} 4 p^{4}, 4 s^{2} 4 p^{2} 4 d^{1}, 4 s^{2} 4 p^{2} 5 s^{1}, 4 s^{2} 4 p^{2} 5 p^{1} \\
4 s^{2} 4 p^{2} 5 d^{1}, 4 s^{2} 4 p^{2} 6 s^{1}\end{array}$ & 106 & 1448 & 1448 \\
\hline Rb I & $\begin{array}{l}4 \mathbf{p}^{6} 5 \mathbf{s}^{1}, 4 p^{6} 5 p^{1}, 4 p^{6} 4 d^{1}, 4 p^{6} 6 s^{1}, 4 p^{6} 6 p^{1} \\
4 p^{6} 5 d^{1}, 4 p^{6} 7 s^{1}, 4 p^{6} 4 f^{1}, 4 p^{6} 7 p^{1}, 4 p^{6} 6 d^{1}\end{array}$ & 17 & 54 & 54 \\
\hline Rb II & $\begin{array}{l}4 \mathbf{p}^{6}, 4 p^{5} 5 s^{1}, 4 p^{5} 4 d^{1}, 4 p^{5} 5 p^{1}, 4 p^{5} 6 s^{1} \\
4 p^{5} 5 d^{1}, 4 p^{5} 6 p^{1}, 4 p^{5} 4 f^{1}, 4 p^{5} 7 s^{1}, 4 p^{5} 6 d^{1} \\
4 p^{5} 5 f^{1}\end{array}$ & 93 & 1251 & 1251 \\
\hline Rb III & $\begin{array}{l}4 \mathbf{s}^{2} 4 \mathbf{p}^{5}, 4 s^{1} 4 p^{6}, 4 s^{2} 4 p^{4} 4 d^{1}, 4 s^{2} 4 p^{4} 5 s^{1}, 4 s^{2} 4 p^{4} 5 p^{1} \\
4 s^{2} 4 p^{4} 6 s^{1}, 4 s^{2} 4 p^{4} 5 d^{1}\end{array}$ & 96 & 1132 & 1132 \\
\hline $\mathrm{Rb}$ IV & $\begin{array}{l}4 \mathbf{s}^{2} 4 \mathbf{p}^{4}, 4 s^{1} 4 p^{5}, 4 s^{2} 4 p^{3} 4 d^{1}, 4 s^{2} 4 p^{3} 5 s^{1}, 4 s^{2} 4 p^{3} 5 p^{1} \\
4 s^{2} 4 p^{3} 5 d^{1}, 4 s^{2} 4 p^{3} 6 s^{1}\end{array}$ & 133 & 2022 & 2022 \\
\hline
\end{tabular}


Table B2. Summary of HULLAC calculations. The last column shows the number of transitions whose upper level is below the ionization potential.

\begin{tabular}{|c|c|c|c|c|}
\hline Ion & Configurations & $N_{\text {level }}$ & $N_{\text {line }}$ & $N_{\text {line }}^{*}$ \\
\hline Sr I & $\begin{array}{l}\mathbf{5 s}^{2}, 5 s^{1} 5 p^{1}, 5 s^{1} 4 d^{1}, 5 s^{1} 6 s^{1}, 4 d^{1} 5 p^{1} \\
5 s^{1} 6 p^{1}, 5 s^{1} 5 d^{1}, 5 p^{2}, 5 s^{1} 7 s^{1}, 5 s^{1} 4 f^{1} \\
5 s^{1} 7 p^{1}, 5 s^{1} 6 d^{1} \\
4 \mathbf{n}^{6} \mathbf{5} \mathbf{s}^{1} 4 n^{6} 4 d^{1} 4 n^{6} 5 p^{1} 4 n^{6} 6 s^{1} 4 n^{6} 5 d^{1}\end{array}$ & 50 & 392 & 365 \\
\hline Sr II & $\begin{array}{l}4 p^{6} 5 s^{1}, 4 p^{6} 4 d^{1}, 4 p^{6} 5 p^{1}, 4 p^{6} 6 s^{1}, 4 p^{6} 5 d^{1} \\
4 p^{6} 6 p^{1}, 4 p^{6} 4 f^{1}, 4 p^{6} 7 s^{1}, 4 p^{6} 6 d^{1}, 4 p^{6} 7 p^{1} \\
4 p^{6} 5 f^{1}, 4 p^{6} 5 g^{1}\end{array}$ & 21 & 69 & 69 \\
\hline Sr III & $\begin{array}{l}4 \mathbf{p}^{6}, 4 p^{5} 4 d^{1}, 4 p^{5} 5 s^{1}, 4 p^{5} 5 p^{1}, 4 p^{5} 5 d^{1} \\
4 p^{5} 6 s^{1}, 4 p^{5} 4 f^{1}, 4 p^{5} 6 p^{1}\end{array}$ & 65 & 634 & 634 \\
\hline Sr IV & $\begin{array}{l}\mathbf{4} \mathbf{s}^{\mathbf{2}} \mathbf{4} \mathbf{p}^{\mathbf{5}}, 4 s^{1} 4 p^{6}, 4 s^{2} 4 p^{4} 4 d^{1}, 4 s^{2} 4 p^{4} 5 s^{1}, 4 s^{2} 4 p^{4} 5 p^{1} \\
4 s^{2} 4 p^{4} 5 d^{1}, 4 s^{2} 4 p^{4} 4 f^{1} \\
\mathbf{4} \mathbf{d}^{1} \mathbf{5} \mathbf{s}^{2}, 5 s^{2} 5 p^{1}, 4 d^{2} 5 s^{1}, 4 d^{1} 5 s^{1} 5 p^{1}, 4 d^{2} 5 p^{1}\end{array}$ & 118 & 2020 & 2020 \\
\hline Y I & $\begin{array}{l}4 d^{3}, 5 s^{2} 6 s^{1}, 4 d^{1} 5 s^{1} 6 s^{1}, 5 p^{2} 5 s^{1}, 5 s^{2} 5 d^{1} \\
5 s^{2} 6 p^{1} \\
\mathbf{5 s}^{2}, 4 d^{1} 5 s^{1}, 4 d^{2}, 5 s^{1} 5 p^{1}, 4 d^{1} 5 p^{1}\end{array}$ & 128 & 2385 & 1958 \\
\hline Y II & $\begin{array}{l}4 d^{1} 6 s^{1}, 5 s^{1} 6 s^{1}, 4 d^{1} 5 d^{1}, 5 p^{2}, 4 d^{1} 6 p^{1} \\
5 s^{1} 5 d^{1}, 5 s^{1} 6 p^{1}, 4 d^{1} 4 f^{1}\end{array}$ & 99 & 1324 & 1324 \\
\hline Y III & $\begin{array}{l}\mathbf{4 p}^{6} \mathbf{4} \mathbf{d}^{1}, 4 p^{6} 5 s^{1}, 4 p^{6} 5 p^{1}, 4 p^{6} 6 s^{1}, 4 p^{6} 5 d^{1} \\
4 p^{6} 6 p^{1}, 4 p^{6} 4 f^{1}, 4 p^{6} 7 s^{1}, 4 p^{6} 6 d^{1}, 4 p^{6} 5 f^{1}\end{array}$ & 17 & 48 & 48 \\
\hline Y IV & $\begin{array}{l}\mathbf{4} \mathbf{p}^{\mathbf{6}}, 4 p^{5} 4 d^{1}, 4 p^{5} 5 s^{1}, 4 p^{5} 5 p^{1}, 4 p^{5} 5 d^{1} \\
4 p^{5} 4 f^{1}, 4 p^{5} 6 s^{1}, 4 p^{5} 6 p^{1} \\
\mathbf{4 d}^{\mathbf{2}} \mathbf{5} \mathbf{s}^{2}, 4 d^{3} 5 s^{1}, 4 d^{2} 5 s^{1} 5 p^{1}, 4 d^{1} 5 s^{2} 5 p^{1}, 4 d^{4}\end{array}$ & 65 & 634 & 634 \\
\hline Zr I & $\begin{array}{l}4 d^{3} 5 p^{1}, 4 d^{2} 5 s^{1} 6 s^{1}, 4 d^{2} 5 s^{1} 6 p^{1}, 4 d^{2} 5 s^{1} 5 d^{1}, 4 d^{3} 6 s^{1} \\
4 d^{2} 5 p^{2}, 4 d^{2} 5 s^{1} 7 p^{1}\end{array}$ & 788 & 76953 & 42680 \\
\hline Zr II & $\begin{array}{l}\mathbf{4} \mathbf{d}^{2} \mathbf{5} \mathbf{s}^{1}, 4 d^{3}, 4 d^{1} 5 s^{2}, 4 d^{2} 5 p^{1}, 4 d^{1} 5 s^{1} 5 p^{1} \\
4 d^{2} 6 s^{1}, 4 d^{2} 5 d^{1}\end{array}$ & 188 & 4452 & 4452 \\
\hline Zr III & $\begin{array}{l}4 \mathbf{d}^{2}, 4 d^{1} 5 s^{1}, 5 s^{2}, 4 d^{1} 5 p^{1}, 5 s^{1} 5 p^{1} \\
4 d^{1} 5 d^{1}, 4 d^{1} 6 s^{1}, 4 d^{1} 6 p^{1}, 4 d^{1} 4 f^{1}\end{array}$ & 84 & 933 & 933 \\
\hline Zr IV & $\begin{array}{l}\mathbf{4} \mathbf{p}^{\mathbf{6}} \mathbf{4 \mathbf { d } ^ { 1 }}, 4 p^{6} 5 s^{1}, 4 p^{6} 5 p^{1}, 4 p^{6} 5 d^{1}, 4 p^{6} 6 s^{1} \\
4 p^{6} 4 f^{1}, 4 p^{6} 6 p^{1}, 4 p^{6} 6 d^{1}\end{array}$ & 14 & 35 & 35 \\
\hline $\mathrm{Nb} \mathrm{I}$ & 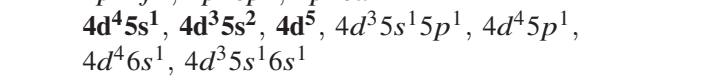 & 649 & 49341 & 20961 \\
\hline Nb II & 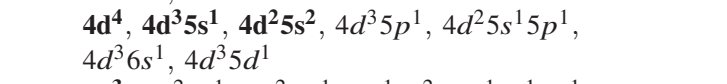 & 487 & 27199 & 27162 \\
\hline Nb III & $\begin{array}{l}4 d^{3}, 4 d^{2} 5 s^{1}, 4 d^{2} 5 p^{1}, 4 d^{1} 5 s^{2}, 4 d^{1} 5 s^{1} 5 p^{1} \\
4 d^{2} 5 d^{1}, 4 d^{2} 6 s^{1}\end{array}$ & 188 & 4452 & 4452 \\
\hline $\mathrm{Nb}$ IV & $\begin{array}{l}4 \mathbf{d}^{2}, 4 d^{1} 5 s^{1}, 4 d^{1} 5 p^{1}, 5 s^{2}, 5 s^{1} 5 p^{1} \\
4 d^{1} 5 d^{1}, 4 d^{1} 6 s^{1}\end{array}$ & 52 & 336 & 336 \\
\hline Mo I & $\begin{array}{l}4 d^{5} 5 \mathbf{s}^{1}, 4 d^{4} 5 s^{2}, 4 d^{6}, 4 d^{5} 5 p^{1}, 4 d^{4} 5 s^{1} 5 p^{1} \\
4 d^{5} 6 s^{1}, 4 d^{5} 5 d^{1}, 4 d^{5} 7 s^{1}, 4 d^{4} 5 s^{1} 6 s^{1}, 4 d^{5} 6 d^{1}\end{array}$ & 1654 & 275258 & 9747 \\
\hline Mo II & $\begin{array}{l}4 \mathbf{d}^{5}, 4 d^{4} 5 s^{1}, 4 d^{4} 5 p^{1}, 4 d^{3} 5 s^{2}, 4 d^{3} 5 s^{1} 5 p^{1} \\
4 d^{4} 6 s^{1}, 4 d^{4} 5 d^{1}\end{array}$ & 851 & 84312 & 66668 \\
\hline Mo III & $\begin{array}{l}4 d^{4}, 4 d^{3} 5 s^{1}, 4 d^{3} 5 p^{1}, 4 d^{2} 5 s^{2}, 4 d^{2} 5 s^{1} 5 p^{1} \\
4 d^{3} 6 s^{1}, 4 d^{3} 5 d^{1}\end{array}$ & 487 & 27199 & 27199 \\
\hline Mo IV & $\begin{array}{l}\mathbf{4 d}^{3}, 4 d^{2} 5 s^{1}, 4 d^{2} 5 p^{1}, 4 d^{1} 5 s^{2}, 4 d^{1} 5 s^{1} 5 p^{1} \\
4 d^{2} 6 s^{1}, 4 d^{2} 5 d^{1}\end{array}$ & 188 & 4452 & 4452 \\
\hline Tc I & $\begin{array}{l}4 d^{5} 5 \mathbf{s}^{2}, 4 d^{6} 5 s^{1}, 4 d^{7}, 4 d^{5} 5 s^{1} 5 p^{1}, 4 d^{6} 5 p^{1} \\
4 d^{5} 5 s^{1} 6 s^{1}, 4 d^{6} 6 s^{1}, 4 d^{5} 5 s^{1} 5 d^{1}, 4 d^{6} 5 d^{1}, 4 d^{6} 6 p^{1}\end{array}$ & 2026 & 440047 & 27092 \\
\hline Tc II & $\begin{array}{l}\mathbf{4 d}^{\mathbf{5}} \mathbf{5} \mathbf{s}^{\mathbf{1}}, \mathbf{4 d}^{\mathbf{6}}, 4 d^{5} 5 p^{1}, 4 d^{4} 5 s^{2}, 4 d^{4} 5 s^{1} 5 p^{1} \\
4 d^{5} 6 s^{1}, 4 d^{5} 5 d^{1}\end{array}$ & 1122 & 138447 & 72563 \\
\hline Tc III & $\begin{array}{l}4 \mathbf{d}^{5}, 4 d^{4} 5 s^{1}, 4 d^{4} 5 p^{1}, 4 d^{3} 5 s^{2}, 4 d^{3} 5 s^{1} 5 p^{1} \\
4 d^{4} 6 s^{1}, 4 d^{4} 5 d^{1}\end{array}$ & 851 & 84312 & 82338 \\
\hline Tc IV & $\begin{array}{l}4 d^{4}, 4 d^{3} 5 s^{1}, 4 d^{3} 5 p^{1}, 4 d^{2} 5 s^{2}, 4 d^{2} 5 s^{1} 5 p^{1} \\
4 d^{3} 6 s^{1}, 4 d^{3} 5 d^{1}\end{array}$ & 487 & 27199 & 27199 \\
\hline
\end{tabular}


Table B2 - continued Summary of HULLAC calculations. The last column shows the number of transitions whose upper level is below the ionization potential.

\begin{tabular}{|c|c|c|c|c|}
\hline Ion & Configurations & $N_{\text {level }}$ & $N_{\text {line }}$ & $N_{\text {line }}^{*}$ \\
\hline Ru I & $\begin{array}{l}\mathbf{4 d}^{7} \mathbf{5} \mathbf{s}^{1}, \mathbf{4 d}^{\mathbf{6}} \mathbf{5} \mathbf{s}^{\mathbf{2}}, \mathbf{4 d}^{\mathbf{8}}, 4 d^{6} 5 s^{1} 5 p^{1}, 4 d^{7} 5 p^{1} \\
4 d^{7} 6 s^{1}, 4 d^{7} 6 p^{1}, 4 d^{7} 5 d^{1}, 4 d^{6} 5 s^{1} 6 s^{1}, 4 d^{6} 5 s^{1} 5 d^{1}\end{array}$ & 1545 & 250476 & 6823 \\
\hline Ru II & $\begin{array}{l}4 \mathbf{d}^{7}, 4 d^{6} 5 s^{1}, 4 d^{5} 5 s^{2}, 4 d^{6} 5 p^{1}, 4 d^{5} 5 s^{1} 5 p^{1} \\
4 d^{6} 6 s^{1}, 4 d^{6} 5 d^{1}, 4 d^{5} 5 s^{1} 6 p^{1}\end{array}$ & 1472 & 213952 & 58450 \\
\hline $\mathrm{Ru}$ III & $4 d^{6}, 4 d^{5} 5 s^{1}, 4 d^{5} 5 p^{1}, 4 d^{5} 5 d^{1}, 4 d^{5} 6 s^{1}$ & 728 & 49066 & 48911 \\
\hline $\mathrm{Ru}$ IV & $\begin{array}{l}4 \mathbf{d}^{5}, 4 d^{4} 5 s^{1}, 4 d^{4} 5 p^{1}, 4 d^{3} 5 s^{2}, 4 d^{3} 5 s^{1} 5 p^{1} \\
4 d^{4} 6 s^{1}, 4 d^{4} 5 d^{1}\end{array}$ & 851 & 84312 & 84312 \\
\hline $\mathrm{Rh} I$ & $\mathbf{4 d}^{\mathbf{8}} \mathbf{5} \mathbf{s}^{\mathbf{1}}, \mathbf{4 d}^{\mathbf{9}}, \mathbf{4 d}^{\mathbf{8}} \mathbf{5} \mathbf{p}^{\mathbf{1}}, 4 d^{7} 5 s^{2}, 4 d^{8} 6 s^{1}$ & 98 & 1321 & 1190 \\
\hline Rh II & $\begin{array}{l}4 \mathbf{d}^{8}, 4 d^{7} 5 s^{1}, 4 d^{7} 5 p^{1}, 4 d^{6} 5 s^{2}, 4 d^{7} 6 s^{1} \\
4 d^{7} 6 p^{1}\end{array}$ & 339 & 12696 & 10992 \\
\hline Rh III & $\begin{array}{l}4 \mathbf{d}^{7}, 4 d^{6} 5 s^{1}, 4 d^{6} 5 p^{1}, 4 d^{5} 5 s^{2}, 4 d^{6} 5 d^{1} \\
4 d^{6} 6 s^{1}, 4 d^{6} 6 p^{1}\end{array}$ & 818 & 76592 & 74318 \\
\hline Rh IV & $\begin{array}{l}\mathbf{4 d}^{\mathbf{6}}, 4 d^{5} 5 s^{1}, 4 d^{5} 5 p^{1}, 4 d^{4} 5 s^{2}, 4 d^{5} 5 d^{1} \\
4 d^{5} 6 s^{1}, 4 d^{5} 6 p^{1}\end{array}$ & 976 & 104622 & 104622 \\
\hline Pd I & $\begin{array}{l}\mathbf{4 d}^{\mathbf{1 0}}, 4 d^{9} 5 s^{1}, 4 d^{8} 5 s^{2}, 4 d^{9} 5 p^{1}, 4 d^{9} 6 s^{1} \\
4 d^{8} 5 s^{1} 5 p^{1}, 4 d^{9} 6 p^{1}, 4 d^{9} 5 d^{1}\end{array}$ & 150 & 2206 & 206 \\
\hline $\mathrm{Pd}$ II & $\begin{array}{l}4 d^{9}, 4 d^{8} 5 s^{1}, 4 d^{8} 5 p^{1}, 4 d^{7} 5 s^{2}, 4 d^{7} 5 s^{1} 5 p^{1} \\
4 d^{8} 6 s^{1}, 4 d^{8} 5 d^{1}, 4 d^{8} 6 p^{1}\end{array}$ & 423 & 18636 & 8018 \\
\hline Pd III & $4 \mathbf{d}^{8}, 4 d^{7} 5 s^{1}, 4 d^{7} 5 p^{1}, 4 d^{7} 6 s^{1}, 4 d^{6} 5 s^{1} 5 p^{1}$ & 555 & 19157 & 15929 \\
\hline Pd IV & $\begin{array}{l}\mathbf{4} \mathbf{d}^{\mathbf{7}}, 4 d^{6} 5 s^{1}, 4 d^{6} 5 p^{1}, 4 d^{6} 5 d^{1}, 4 d^{6} 6 s^{1} \\
4 d^{6} 6 p^{1} \\
\mathbf{4 d}^{\mathbf{1 0}} \mathbf{5} \mathbf{s}^{\mathbf{1}}, \mathbf{4 d}^{\mathbf{1 0}} \mathbf{5} \mathbf{p}^{\mathbf{1}}, \mathbf{4 d}^{\mathbf{9}} \mathbf{5} \mathbf{s}^{\mathbf{2}}, 4 d^{10} 6 s^{1}, 4 d^{10} 6 p^{1}\end{array}$ & 781 & 70102 & 70102 \\
\hline $\mathrm{Ag} \mathrm{I}$ & $\begin{array}{l}4 d^{10} 5 d^{1}, 4 d^{10} 7 s^{1}, 4 d^{10} 7 p^{1}, 4 d^{10} 6 d^{1}, 4 d^{10} 4 f^{1}, \\
4 d^{10} 8 s^{1}\end{array}$ & 18 & 60 & 60 \\
\hline $\mathrm{Ag}$ II & $\begin{array}{l}\mathbf{4 d ^ { 1 0 }}, 4 d^{9} 5 s^{1}, 4 d^{9} 5 p^{1}, 4 d^{8} 5 s^{2}, 4 d^{9} 6 s^{1} \\
4 d^{9} 5 d^{1}, 4 d^{8} 5 s^{1} 5 p^{1}, 4 d^{9} 6 p^{1}\end{array}$ & 150 & 2206 & 642 \\
\hline Ag III & $\begin{array}{l}\mathbf{4 d}^{9}, 4 d^{8} 5 s^{1}, 4 d^{8} 5 p^{1}, 4 d^{7} 5 s^{2}, 4 d^{8} 6 s^{1} \\
4 d^{8} 5 d^{1}, 4 d^{8} 6 p^{1}\end{array}$ & 210 & 5764 & 5764 \\
\hline Ag IV & $\begin{array}{l}4 \mathbf{d}^{8}, 4 d^{7} 5 s^{1}, 4 d^{7} 5 p^{1}, 4 d^{6} 5 s^{2}, 4 d^{7} 6 s^{1} \\
4 d^{7} 5 d^{1}, 4 d^{7} 6 p^{1}\end{array}$ & 507 & 29606 & 29606 \\
\hline Cd I & $\begin{array}{l}\mathbf{4 d}^{\mathbf{1 0}} \mathbf{5} \mathbf{s}^{2}, 4 d^{10} 5 s^{1} 5 p^{1}, 4 d^{10} 5 s^{1} 6 s^{1}, 4 d^{10} 5 s^{1} 6 p^{1}, 4 d^{10} 5 s^{1} 5 d^{1} \\
4 d^{10} 5 s^{1} 7 s^{1}, 4 d^{10} 5 s^{1} 7 p^{1}, 4 d^{10} 5 s^{1} 6 d^{1}, 4 d^{10} 5 s^{1} 4 f^{1} \\
\mathbf{4 d}^{10} \mathbf{5} \mathbf{s}^{1}, 4 d^{10} 5 p^{1}, 4 d^{9} 5 s^{2}, 4 d^{10} 6 s^{1}, 4 d^{10} 5 d^{1}\end{array}$ & 29 & 130 & 130 \\
\hline $\mathrm{Cd}$ II & $\begin{array}{l}4 d^{10} 6 p^{1}, 4 d^{9} 5 s^{1} 5 p^{1}, 4 d^{10} 7 s^{1}, 4 d^{10} 4 f^{1}, 4 d^{10} 6 d^{1}, \\
4 d^{10} 7 p^{1}\end{array}$ & 40 & 195 & 156 \\
\hline Cd III & $\begin{array}{l}\mathbf{4 \mathbf { d } ^ { 1 0 }}, 4 d^{9} 5 s^{1}, 4 d^{9} 5 p^{1}, 4 d^{8} 5 s^{2}, 4 d^{9} 5 d^{1} \\
4 d^{9} 6 s^{1}\end{array}$ & 48 & 258 & 258 \\
\hline Cd IV & $\begin{array}{l}4 \mathbf{d}^{9}, 4 d^{8} 5 s^{1}, 4 d^{8} 5 p^{1}, 4 d^{7} 5 s^{2}, 4 d^{8} 5 d^{1} \\
4 d^{8} 6 s^{1}\end{array}$ & 165 & 2882 & 2882 \\
\hline In I & $\begin{array}{l}\mathbf{5 s}^{2} 5 \mathbf{p}^{1}, 5 s^{2} 6 s^{1}, 5 s^{2} 6 p^{1}, 5 s^{2} 5 d^{1}, 5 s^{1} 5 p^{2} \\
5 s^{2} 7 s^{1}, 5 s^{2} 7 p^{1}, 5 s^{2} 6 d^{1}, 5 s^{2} 4 f^{1}\end{array}$ & 22 & 85 & 54 \\
\hline In II & $\begin{array}{l}\mathbf{5 s}^{2}, 5 s^{1} 5 p^{1}, 5 s^{1} 6 s^{1}, 5 s^{1} 5 d^{1}, 5 p^{2} \\
5 s^{1} 6 p^{1}, 5 s^{1} 7 s^{1}, 5 s^{1} 4 f^{1}, 5 s^{1} 6 d^{1}, 5 s^{1} 7 p^{1}\end{array}$ & 34 & 181 & 181 \\
\hline In III & $\begin{array}{l}\mathbf{4 d ^ { 1 0 }} 5 \mathbf{s}^{1}, 4 d^{10} 5 p^{1}, 4 d^{9} 5 s^{2}, 4 d^{10} 6 s^{1}, 4 d^{10} 5 d^{1} \\
4 d^{10} 6 p^{1}, 4 d^{9} 5 s^{1} 5 p^{1}, 4 d^{10} 4 f^{1}\end{array}$ & 35 & 120 & 120 \\
\hline In IV & $\begin{array}{l}\mathbf{4 d}^{\mathbf{1 0}}, 4 d^{9} 5 s^{1}, 4 d^{9} 5 p^{1}, 4 d^{8} 5 s^{2}, 4 d^{9} 5 d^{1} \\
4 d^{9} 6 s^{1}\end{array}$ & 48 & 258 & 258 \\
\hline
\end{tabular}


Table B3. Summary of HULLAC calculations. The last column shows the number of transitions whose upper level is below the ionization potential.

\begin{tabular}{|c|c|c|c|c|}
\hline Ion & Configurations & $N_{\text {level }}$ & $N_{\text {line }}$ & $N_{\text {line }}^{*}$ \\
\hline Sn I & $\begin{array}{l}\mathbf{5 s}^{2} 5 \mathbf{p}^{2}, 5 s^{2} 5 p^{1} 6 s^{1}, 5 s^{1} 5 p^{3}, 5 s^{2} 5 p^{1} 6 p^{1}, 5 s^{2} 5 p^{1} 5 d^{1}, \\
5 s^{2} 5 p^{1} 7 s^{1}, 5 s^{2} 5 p^{1} 7 p^{1}, 5 s^{2} 5 p^{1} 6 d^{1}, 5 s^{2} 5 p^{1} 4 f^{1}, 5 s^{2} 5 p^{1} 8 s^{1}, \\
5 s^{2} 5 p^{1} 7 d^{1} \\
\mathbf{5 s}^{\mathbf{2}} \mathbf{5} \mathbf{p}^{1}, 5 s^{1} 5 p^{2}, 5 s^{2} 6 s^{1}, 5 s^{2} 5 d^{1}, 5 s^{2} 6 p^{1},\end{array}$ & 95 & 1326 & 464 \\
\hline Sn II & $\begin{array}{l}5 s^{2} 7 s^{1}, 5 s^{2} 4 f^{1}, 5 s^{2} 6 d^{1}, 5 s^{2} 7 p^{1}, 5 s^{2} 8 s^{1} \\
5 s^{2} 5 f^{1}\end{array}$ & 25 & 104 & 104 \\
\hline Sn III & $\begin{array}{l}\mathbf{4 d ^ { 1 0 }} 5 \mathbf{s}^{2}, 4 d^{10} 5 s^{1} 5 p^{1}, 4 d^{10} 5 p^{2}, 4 d^{10} 5 s^{1} 6 s^{1}, 4 d^{10} 5 s^{1} 5 d^{1}, \\
4 d^{10} 5 s^{1} 6 p^{1}, 4 d^{10} 4 f^{1} 5 s^{1}, 4 d^{10} 5 s^{1} 7 s^{1}, 4 d^{10} 5 s^{1} 6 d^{1}, 4 d^{10} 5 s^{1} 7 p^{1} \\
\mathbf{4 d}^{10} 5 \mathbf{s}^{1}, 4 d^{10} 5 p^{1}, 4 d^{10} 5 d^{1}, 4 d^{9} 5 s^{2}, 4 d^{10} 6 s^{1}\end{array}$ & 34 & 181 & 181 \\
\hline Sn IV & $\begin{array}{l}4 d^{10} 6 p^{1}, 4 d^{10} 4 f^{1}, 4 d^{10} 6 d^{1}, 4 d^{10} 7 s^{1}, 4 d^{10} 5 g^{1} \\
4 d^{9} 5 s^{1} 5 p^{1} \\
\mathbf{5 p}^{3}, 5 p^{2} 6 s^{1}, 5 p^{2} 6 p^{1}, 5 p^{2} 5 d^{1}, 5 p^{2} 7 s^{1}\end{array}$ & 40 & 198 & 198 \\
\hline $\mathrm{Sb} \mathrm{I}$ & $\begin{array}{l}5 p^{2} 6 d^{1}, 5 p^{2} 7 p^{1}, 5 p^{2} 4 f^{1}, 5 p^{2} 8 s^{1}, 5 p^{2} 8 p^{1} \\
5 p^{2} 7 d^{1}\end{array}$ & 206 & 6684 & 794 \\
\hline Sb II & $\begin{array}{l}5 s^{2} 5 \mathbf{p}^{2}, 5 s^{1} 5 p^{3}, 5 s^{2} 5 p^{1} 6 s^{1}, 5 s^{2} 5 p^{1} 5 d^{1}, 5 s^{2} 5 p^{1} 6 p^{1} \\
5 s^{2} 5 p^{1} 7 s^{1}, 5 s^{2} 5 p^{1} 6 d^{1}, 5 s^{2} 5 p^{1} 4 f^{1}, 5 p^{4}\end{array}$ & 74 & 809 & 678 \\
\hline Sb III & $\begin{array}{l}\mathbf{5} \mathbf{s}^{2} 5 \mathbf{p}^{1}, 5 s^{1} 5 p^{2}, 5 s^{2} 6 s^{1}, 5 s^{2} 5 d^{1}, 5 s^{2} 6 p^{1} \\
5 s^{2} 4 f^{1}, 5 s^{2} 7 s^{1}, 5 s^{2} 6 d^{1}, 5 s^{2} 8 s^{1}\end{array}$ & 21 & 65 & 65 \\
\hline Sb IV & $\begin{array}{l}5 s^{2}, 5 s^{1} 5 p^{1}, 5 p^{2}, 5 s^{1} 5 d^{1}, 5 s^{1} 6 s^{1} \\
5 s^{1} 6 p^{1}, 5 s^{1} 4 f^{1}, 5 s^{1} 6 d^{1}, 5 s^{1} 7 s^{1}\end{array}$ & 30 & 131 & 131 \\
\hline Te I & $\begin{array}{l}\mathbf{5 p}^{4}, 5 p^{3} 6 s^{1}, 5 p^{3} 6 p^{1}, 5 p^{3} 5 d^{1}, 5 p^{3} 7 s^{1} \\
5 p^{3} 7 p^{1}, 5 p^{3} 6 d^{1}, 5 p^{3} 4 f^{1}, 5 p^{3} 8 s^{1}, 5 p^{3} 7 d^{1}\end{array}$ & 245 & 8313 & 1546 \\
\hline Te II & $\begin{array}{l}5 \mathbf{s}^{2} 5 \mathbf{p}^{3}, 5 s^{1} 5 p^{4}, 5 s^{2} 5 p^{2} 6 s^{1}, 5 s^{2} 5 p^{2} 5 d^{1}, 5 s^{2} 5 p^{2} 6 p^{1} \\
5 s^{2} 5 p^{2} 7 s^{1}, 5 s^{2} 5 p^{2} 6 d^{1}, 5 s^{2} 5 p^{2} 4 f^{1}, 5 s^{2} 5 p^{2} 7 p^{1}, 5 s^{2} 5 p^{2} 8 s^{1}\end{array}$ & 165 & 4238 & 3870 \\
\hline Te III & $\begin{array}{l}\mathbf{5 s}^{2} 5 \mathbf{p}^{2}, 5 s^{1} 5 p^{3}, 5 s^{2} 5 p^{1} 5 d^{1}, 5 s^{2} 5 p^{1} 6 s^{1}, 5 s^{2} 5 p^{1} 6 p^{1} \\
5 s^{2} 5 p^{1} 6 d^{1}, 5 s^{2} 5 p^{1} 7 s^{1}\end{array}$ & 57 & 419 & 419 \\
\hline Te IV & $\begin{array}{l}\mathbf{5 s}^{2} \mathbf{5} \mathbf{p}^{1}, 5 s^{1} 5 p^{2}, 5 s^{2} 5 d^{1}, 5 s^{2} 6 s^{1}, 5 s^{2} 6 p^{1} \\
5 s^{2} 6 d^{1}, 5 s^{2} 7 s^{1} \\
\mathbf{5 s}^{2} 5 \mathbf{p}^{5}, 5 s^{2} 5 p^{4} 6 s^{1}, 5 s^{2} 5 p^{4} 6 p^{1}, 5 s^{2} 5 p^{4} 5 d^{1}, 5 s^{2} 5 p^{4} 7 s^{1}\end{array}$ & 18 & 48 & 48 \\
\hline I I & $\begin{array}{l}5 s^{2} 5 p^{4} 7 p^{1}, 5 s^{2} 5 p^{4} 6 d^{1}, 5 s^{2} 5 p^{4} 4 f^{1}, 5 s^{2} 5 p^{4} 8 s^{1}, 5 s^{2} 5 p^{4} 8 p^{1}, \\
5 s^{2} 5 p^{4} 7 d^{1} \\
\mathbf{5 s}^{2} 5 p^{4}, 5 s^{2} 5 p^{3} 6 s^{1}, 5 s^{1} 5 p^{5}, 5 s^{2} 5 p^{3} 5 d^{1}, 5 s^{2} 5 p^{3} 6 p^{1}\end{array}$ & 203 & 6432 & 3000 \\
\hline I II & $\begin{array}{l}5 s^{2} 5 p^{3} 7 s^{1}, 5 s^{2} 5 p^{3} 6 d^{1}, 5 s^{2} 5 p^{3} 4 f^{1}, 5 s^{2} 5 p^{3} 7 p^{1}, 5 s^{2} 5 p^{3} 8 s^{1} \\
5 s^{2} 5 p^{3} 7 d^{1}, 5 s^{2} 5 p^{3} 5 f^{1}\end{array}$ & 289 & 11510 & 8251 \\
\hline I III & $\begin{array}{l}\mathbf{5 s}^{2} 5 \mathbf{p}^{3}, 5 s^{1} 5 p^{4}, 5 s^{2} 5 p^{2} 5 d^{1}, 5 s^{2} 5 p^{2} 6 s^{1}, 5 s^{2} 5 p^{2} 7 s^{1} \\
5 s^{2} 5 p^{2} 6 d^{1}, 5 s^{2} 5 p^{2} 8 s^{1}, 5 s^{2} 5 p^{2} 7 d^{1}\end{array}$ & 121 & 431 & 431 \\
\hline I IV & $\begin{array}{l}\mathbf{5 s}^{2} 5 \mathbf{p}^{2}, 5 s^{1} 5 p^{3}, 5 s^{2} 5 p^{1} 5 d^{1}, 5 s^{2} 5 p^{1} 6 s^{1}, 5 s^{2} 5 p^{1} 6 d^{1} \\
5 s^{2} 5 p^{1} 7 s^{1}, 5 s^{2} 5 p^{1} 7 d^{1}, 5 s^{2} 5 p^{1} 8 s^{1}\end{array}$ & 63 & 179 & 179 \\
\hline Xe I & $\begin{array}{l}\mathbf{5 p}^{6}, 5 p^{5} 6 s^{1}, 5 p^{5} 6 p^{1}, 5 p^{5} 5 d^{1}, 5 p^{5} 7 s^{1} \\
5 p^{5} 7 p^{1}, 5 p^{5} 6 d^{1}, 5 p^{5} 8 s^{1}, 5 p^{5} 4 f^{1}, 5 p^{5} 7 d^{1}\end{array}$ & 81 & 951 & 715 \\
\hline Xe II & $\begin{array}{l}\mathbf{5 s}^{2} 5 \mathbf{p}^{5}, 5 s^{1} 5 p^{6}, 5 s^{2} 5 p^{4} 6 s^{1}, 5 s^{2} 5 p^{4} 5 d^{1}, 5 s^{2} 5 p^{4} 6 p^{1} \\
5 s^{2} 5 p^{4} 7 s^{1}, 5 s^{2} 5 p^{4} 6 d^{1}, 5 s^{2} 5 p^{4} 4 f^{1}, 5 s^{2} 5 p^{4} 7 p^{1}, 5 s^{2} 5 p^{4} 8 s^{1}\end{array}$ & 155 & 3685 & 3393 \\
\hline Xe III & $\begin{array}{l}\mathbf{5 s}^{2} 5 \mathbf{p}^{4}, 5 s^{1} 5 p^{5}, 5 s^{2} 5 p^{3} 5 d^{1}, 5 s^{2} 5 p^{3} 6 s^{1}, 5 s^{2} 5 p^{3} 6 p^{1} \\
5 s^{2} 5 p^{3} 4 f^{1}, 5 s^{2} 5 p^{3} 6 d^{1}, 5 s^{2} 5 p^{3} 7 s^{1}, 5 s^{2} 5 p^{3} 5 f^{1}, 5 p^{6}\end{array}$ & 214 & 6052 & 6024 \\
\hline $\mathrm{Xe}$ IV & $\begin{array}{l}\mathbf{5 s}^{2} 5 \mathbf{p}^{3}, 5 s^{1} 5 p^{4}, 5 s^{2} 5 p^{2} 5 d^{1}, 5 s^{2} 5 p^{2} 6 s^{1}, 5 s^{2} 5 p^{2} 4 f^{1} \\
5 s^{2} 5 p^{2} 6 p^{1}\end{array}$ & 100 & 1480 & 1480 \\
\hline Cs I & $\begin{array}{l}\mathbf{5 p}^{6} \mathbf{6} \mathbf{s}^{1}, 5 p^{6} 6 p^{1}, 5 p^{6} 5 d^{1}, 5 p^{6} 7 s^{1}, 5 p^{6} 7 p^{1} \\
5 p^{6} 6 d^{1}, 5 p^{6} 8 s^{1}, 5 p^{6} 4 f^{1}, 5 p^{6} 8 p^{1}, 5 p^{6} 7 d^{1} \\
\mathbf{5 p}^{6}, 5 p^{5} 6 s^{1}, 5 p^{5} 5 d^{1}, 5 p^{5} 6 p^{1}, 5 p^{5} 7 s^{1}\end{array}$ & 17 & 54 & 54 \\
\hline Cs II & $\begin{array}{l}5 p^{5} 6 d^{1}, 5 p^{5} 4 f^{1}, 5 p^{5} 7 p^{1}, 5 p^{5} 8 s^{1}, 5 p^{5} 5 f^{1} \\
5 p^{5} 7 d^{1}, 5 p^{5} 8 p^{1} \\
\mathbf{5 s}^{2} 5 \mathbf{p}^{5}, 5 s^{1} 5 p^{6}, 5 s^{2} 5 p^{4} 5 d^{1}, 5 s^{2} 5 p^{4} 6 s^{1}, 5 s^{2} 5 p^{4} 6 p^{1}\end{array}$ & 103 & 1569 & 1569 \\
\hline Cs III & $\begin{array}{l}5 s^{2} 5 p^{4} 4 f^{1}, 5 s^{2} 5 p^{4} 6 d^{1}, 5 s^{2} 5 p^{4} 7 s^{1}, 5 s^{2} 5 p^{4} 7 p^{1}, 5 s^{2} 5 p^{4} 5 f^{1}, \\
5 s^{2} 5 p^{4} 8 s^{1}\end{array}$ & 185 & 4932 & 4932 \\
\hline Cs IV & $\begin{array}{l}\mathbf{5 s}^{2} 5 \mathbf{p}^{4}, 5 s^{1} 5 p^{5}, 5 s^{2} 5 p^{3} 5 d^{1}, 5 s^{2} 5 p^{3} 6 s^{1}, 5 s^{2} 5 p^{3} 6 d^{1} \\
5 s^{2} 5 p^{3} 7 s^{1}, 5 s^{2} 5 p^{3} 7 d^{1}, 5 s^{2} 5 p^{3} 8 s^{1}\end{array}$ & 153 & 410 & 410 \\
\hline
\end{tabular}


Table B3 - continued Summary of HULLAC calculations. The last column shows the number of transitions whose upper level is below the ionization potential.

\begin{tabular}{|c|c|c|c|c|}
\hline Ion & Configurations & $N_{\text {level }}$ & $N_{\text {line }}$ & $N_{\text {line }}^{*}$ \\
\hline $\mathrm{Ba} I$ & $\begin{array}{l}\mathbf{6 s}^{\mathbf{2}}, 6 s^{1} 5 d^{1}, 6 s^{1} 6 p^{1}, 5 d^{2}, 5 d^{1} 6 p^{1}, \\
6 s^{1} 7 s^{1}, 6 s^{1} 6 d^{1}, 6 s^{1} 7 p^{1}, 5 d^{1} 7 s^{1}, 6 s^{1} 8 s^{1}, \\
6 p^{2}, 6 s^{1} 4 f^{1}, 6 s^{1} 7 d^{1} \\
\mathbf{5 p}^{6} \mathbf{6} \mathbf{s}^{1}, 5 p^{6} 5 d^{1}, 5 p^{6} 6 p^{1}, 5 p^{6} 7 s^{1}, 5 p^{6} 6 d^{1} .\end{array}$ & 59 & 516 & 394 \\
\hline Ba II & $5 p^{6} 4 f^{1}, 5 p^{6} 7 p^{1}, 5 p^{6} 5 f^{1}, 5 p^{6} 8 s^{1}, 5 p^{6} 7 d^{1}$ & 19 & 63 & 63 \\
\hline Ba III & $\begin{array}{l}5 \mathbf{p}^{6}, 5 p^{5} 5 d^{1}, 5 p^{5} 6 s^{1}, 5 p^{5} 4 f^{1}, 5 p^{5} 6 p^{1} \\
5 p^{5} 6 d^{1}, 5 p^{5} 7 s^{1}, 5 p^{5} 5 f^{1}, 5 p^{5} 7 p^{1}, 5 p^{5} 7 d^{1} \\
5 p^{5} 8 s^{1}\end{array}$ & 93 & 1251 & 1251 \\
\hline $\mathrm{Ba}$ IV & $\begin{array}{l}\mathbf{5 s}^{2} \mathbf{5} \mathbf{p}^{\mathbf{5}}, 5 s^{1} 5 p^{6}, 5 s^{2} 5 p^{4} 5 d^{1}, 5 s^{2} 5 p^{4} 6 s^{1}, 5 s^{2} 5 p^{4} 6 p^{1} \\
\mathbf{5 d}^{2} \mathbf{6} \mathbf{s}^{1}, 5 d^{1} 6 s^{2}, 5 d^{3}, 5 d^{1} 6 s^{1} 6 p^{1}, 4 f^{1} 6 s^{2}\end{array}$ & 60 & 574 & 574 \\
\hline La I & $\begin{array}{l}6 s^{2} 6 p^{1}, 5 d^{2} 6 p^{1}, 4 f^{1} 5 d^{1} 6 s^{1}, 4 f^{1} 6 s^{1} 6 p^{1}, 5 d^{2} 7 s^{1} \\
5 d^{1} 6 s^{1} 7 s^{1}, 5 d^{2} 6 d^{1}, 5 d^{2} 7 p^{1}, 4 f^{1} 5 d^{2}, 5 d^{1} 6 s^{1} 7 p^{1} \\
6 s^{2} 8 p^{1}\end{array}$ & 414 & 20274 & 8229 \\
\hline La II & 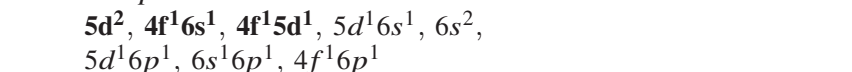 & 66 & 553 & 553 \\
\hline La III & $\begin{array}{l}\mathbf{5}^{\mathbf{6}} \mathbf{5} \mathbf{d}^{1}, \mathbf{5}^{\mathbf{6}} \mathbf{4} \mathbf{f}^{1}, 5 p^{6} 6 s^{1}, 5 p^{6} 6 p^{1}, 5 p^{6} 7 s^{1} \\
5 p^{6} 6 d^{1}, 5 p^{6} 5 f^{1}, 5 p^{6} 7 p^{1}, 5 p^{6} 8 s^{1}\end{array}$ & 15 & 36 & 36 \\
\hline La IV & $\begin{array}{l}\mathbf{5 p}^{6}, 5 p^{5} 4 f^{1}, 5 p^{5} 5 d^{1}, 5 p^{5} 6 s^{1}, 5 p^{5} 6 p^{1} \\
5 p^{5} 6 d^{1}, 5 p^{5} 7 s^{1}\end{array}$ & 55 & 422 & 422 \\
\hline Ce I & $\begin{array}{l}4 \mathbf{f}^{2} \mathbf{6} \mathbf{s}^{2}, 4 f^{1} 5 d^{1} 6 s^{2}, 4 f^{1} 5 d^{2} 6 s^{1}, 4 f^{2} 5 d^{1} 6 s^{1}, 4 f^{1} 5 d^{1} 6 s^{1} 6 p^{1} \\
4 f^{1} 5 d^{3}, 4 f^{1} 6 s^{2} 6 p^{1}, 4 f^{2} 6 s^{1} 6 p^{1}, 4 f^{1} 5 d^{2} 6 p^{1}, 4 f^{2} 5 d^{2}\end{array}$ & 1920 & 293131 & 150839 \\
\hline Ce II & $\begin{array}{l}\mathbf{5} \mathbf{d}^{2} \mathbf{4} \mathbf{f}^{1}, \mathbf{4} \mathbf{f}^{\mathbf{2}} \mathbf{6} \mathbf{s}^{\mathbf{1}}, \mathbf{4} \mathbf{f}^{2} \mathbf{5} \mathbf{d}^{\mathbf{1}}, 4 f^{1} 5 d^{1} 6 s^{1}, 4 f^{1} 6 s^{2} \\
4 f^{1} 5 d^{1} 6 p^{1}, 4 f^{2} 6 p^{1}, 4 f^{1} 6 s^{1} 6 p^{1} \\
\mathbf{4 f}^{2}, 4 f^{1} 5 d^{1}, 4 f^{1} 6 s^{1}, 5 d^{2}, 4 f^{1} 6 p^{1}\end{array}$ & 459 & 21239 & 21239 \\
\hline Ce III & $\begin{array}{l}5 d^{1} 6 s^{1}, 4 f^{1} 6 d^{1}, 4 f^{1} 7 s^{1}, 5 d^{1} 6 p^{1}, 4 f^{1} 5 f^{1}, \\
4 f^{1} 7 p^{1}, 4 f^{1} 8 s^{1}, 4 f^{1} 7 d^{1}, 4 f^{1} 6 f^{1}, 4 f^{1} 5 g^{1} \\
6 p^{2}, 5 d^{1} 6 d^{1}\end{array}$ & 237 & 6244 & 5556 \\
\hline Ce IV & $\begin{array}{l}\mathbf{5 p}^{6} \mathbf{4} \mathbf{f}^{1}, 5 p^{6} 5 d^{1}, 5 p^{6} 6 s^{1}, 5 p^{6} 6 p^{1}, 5 p^{6} 6 d^{1} \\
5 p^{6} 7 s^{1}\end{array}$ & 10 & 16 & 16 \\
\hline Pr I & $\begin{array}{l}\mathbf{4} \mathbf{f}^{\mathbf{3}} \mathbf{6} \mathbf{s}^{\mathbf{2}}, \mathbf{4} \mathbf{f}^{\mathbf{3}} \mathbf{6} \mathbf{s}^{\mathbf{1}} \mathbf{5} \mathbf{d}^{\mathbf{1}}, \mathbf{4} \mathbf{f}^{\mathbf{3}} \mathbf{6} \mathbf{s}^{\mathbf{1}} \mathbf{6} \mathbf{p}^{\mathbf{1}}, \mathbf{4} \mathbf{f}^{\mathbf{3}} \mathbf{6} \mathbf{s}^{\mathbf{1}} \mathbf{7} \mathbf{s}^{\mathbf{1}}, \mathbf{4} \mathbf{f}^{\mathbf{3}} \mathbf{6} \mathbf{s}^{\mathbf{1}} \mathbf{8} \mathbf{s}^{\mathbf{1}} \\
4 f^{2} 6 s^{2} 5 d^{1}, 4 f^{2} 6 s^{2} 6 p^{1}, 4 f^{2} 5 d^{2} 6 s^{1}, 4 f^{2} 5 d^{2} 6 p^{1}, 4 f^{2} 5 d^{1} 6 s^{1} 6 p^{1}\end{array}$ & 6516 & 2715879 & 663287 \\
\hline Pr II & $\begin{array}{l}\mathbf{4 f ^ { 3 }} \mathbf{6} \mathbf{s}^{1}, \mathbf{4} \mathbf{f}^{3} \mathbf{5} \mathbf{d}^{1}, 4 f^{2} 5 d^{2}, 4 f^{2} 5 d^{1} 6 s^{1}, 4 f^{3} 6 p^{1} \\
4 f^{2} 5 d^{1} 6 p^{1}\end{array}$ & 2007 & 364325 & 346451 \\
\hline Pr III & $\begin{array}{l}4 \mathbf{f}^{3}, 4 f^{2} 5 d^{1}, 4 f^{2} 6 s^{1}, 4 f^{2} 6 p^{1}, 4 f^{1} 5 d^{2} \\
4 f^{1} 5 d^{1} 6 s^{1}, 4 f^{2} 7 s^{1}, 4 f^{2} 6 d^{1}, 4 f^{2} 5 f^{1}, 4 f^{2} 8 s^{1}\end{array}$ & 653 & 42001 & 41920 \\
\hline Pr IV & $\begin{array}{l}\mathbf{4 \mathbf { f } ^ { 2 }}, 4 f^{1} 5 d^{1}, 4 f^{1} 6 s^{1}, 4 f^{1} 6 p^{1}, 5 d^{2} \\
4 f^{1} 6 d^{1}, 5 d^{1} 6 p^{1}\end{array}$ & 90 & 926 & 926 \\
\hline $\mathrm{Nd} \mathrm{I}$ & $\begin{array}{l}\mathbf{4 f}^{\mathbf{4}} \mathbf{6} \mathbf{s}^{\mathbf{2}}, \mathbf{4} \mathbf{f}^{\mathbf{4}} \mathbf{6} \mathbf{s}^{\mathbf{1}} \mathbf{5} \mathbf{d}^{\mathbf{1}}, \mathbf{4} \mathbf{f}^{\mathbf{4}} \mathbf{6} \mathbf{s}^{\mathbf{1}} \mathbf{6} \mathbf{p}^{\mathbf{1}}, \mathbf{4} \mathbf{f}^{\mathbf{4}} \mathbf{6} \mathbf{s}^{\mathbf{1}} \mathbf{7} \mathbf{s}^{\mathbf{1}}, \mathbf{4} \mathbf{f}^{\mathbf{4}} \mathbf{6} \mathbf{s}^{\mathbf{1}} \mathbf{8} \mathbf{s}^{\mathbf{1}}, \\
4 f^{3} 5 d^{1} 6 s^{2}, 4 f^{3} 5 d^{2} 6 s^{1}, 4 f^{3} 5 d^{1} 6 s^{1} 6 p^{1}\end{array}$ & 12215 & 11784658 & 371432 \\
\hline $\mathrm{Nd}$ II & 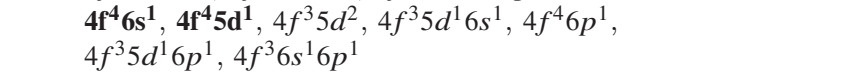 & 6888 & 3947992 & 2281283 \\
\hline Nd III & $\begin{array}{l}\mathbf{4} \mathbf{f}^{4}, 4 f^{3} 5 d^{1}, 4 f^{3} 6 s^{1}, 4 f^{3} 6 p^{1}, 4 f^{2} 5 d^{2} \\
4 f^{2} 5 d^{1} 6 s^{1}, 4 f^{2} 5 d^{1} 6 p^{1}, 4 f^{2} 6 s^{1} 6 p^{1}\end{array}$ & 2252 & 458161 & 225413 \\
\hline $\mathrm{Nd}$ IV & $\begin{array}{l}\mathbf{4 f ^ { 3 }}, 4 f^{2} 5 d^{1}, 4 f^{2} 6 s^{1}, 4 f^{2} 6 p^{1}, 4 f^{1} 5 d^{2} \\
4 f^{1} 5 d^{1} 6 s^{1}, 4 f^{1} 5 d^{1} 6 p^{1}\end{array}$ & 474 & 23864 & 15463 \\
\hline Pm I & $\begin{array}{l}\mathbf{4 f}^{\mathbf{5}} \mathbf{6} \mathbf{s}^{\mathbf{2}}, \mathbf{4} \mathbf{f}^{5} \mathbf{6 s}^{\mathbf{1}} \mathbf{5} \mathbf{d}^{\mathbf{1}}, \mathbf{4 f}^{\mathbf{5}} \mathbf{6} \mathbf{s}^{\mathbf{1}} \mathbf{6} \mathbf{p}^{\mathbf{1}}, \mathbf{4} \mathbf{f}^{\mathbf{5}} \mathbf{6} \mathbf{s}^{\mathbf{1}} \mathbf{7} \mathbf{s}^{\mathbf{1}}, 4 f^{4} 6 s^{2} 5 d^{1}, \\
4 f^{4} 6 s^{1} 5 d^{2}\end{array}$ & 16294 & 17038373 & 321675 \\
\hline Pm II & $\begin{array}{l}\mathbf{4 f ^ { 5 }} \mathbf{6} \mathbf{s}^{1}, 4 f^{5} 5 d^{1}, 4 f^{5} 6 p^{1}, 4 f^{4} 6 s^{1} 6 p^{1}, 4 f^{4} 6 s^{1} 5 d^{1} \\
4 f^{4} 5 d^{1} 6 p^{1}\end{array}$ & 12372 & 9176295 & 3243150 \\
\hline Pm III & $4 \mathbf{f}^{5}, 4 f^{4} 5 d^{1}, 4 f^{4} 6 s^{1}, 4 f^{4} 6 p^{1}$ & 1994 & 320633 & 317305 \\
\hline Pm IV & $4 \mathbf{f}^{4}, 4 f^{3} 5 d^{1}, 4 f^{3} 6 s^{1}, 4 f^{3} 6 p^{1}$ & 817 & 57765 & 57765 \\
\hline
\end{tabular}


Table B4. Summary of HULLAC calculations. The last column shows the number of transitions whose upper level is below the ionization potential.

\begin{tabular}{|c|c|c|c|c|}
\hline Ion & Configurations & $N_{\text {level }}$ & $N_{\text {line }}$ & $N_{\text {line }}^{*}$ \\
\hline Sm I & $\begin{array}{l}\mathbf{4} \mathbf{f}^{\mathbf{6}} \mathbf{6} \mathbf{s}^{\mathbf{2}}, \mathbf{4} \mathbf{f}^{\mathbf{6}} \mathbf{6} \mathbf{s}^{\mathbf{1}} \mathbf{5} \mathbf{d}^{\mathbf{1}}, \mathbf{4} \mathbf{f}^{\mathbf{6}} \mathbf{6} \mathbf{s}^{\mathbf{1}} \mathbf{6} \mathbf{p}^{\mathbf{1}}, \mathbf{4} \mathbf{f}^{\mathbf{6}} \mathbf{6} \mathbf{s}^{\mathbf{1}} 7 \mathbf{s}^{\mathbf{1}}, 4 f^{5} 5 d^{1} 6 s^{2}, \\
4 f^{5} 5 d^{2} 6 s^{1}\end{array}$ & 28221 & 43903718 & 54329 \\
\hline Sm II & $\mathbf{4} \mathbf{f}^{\mathbf{6}} \mathbf{6} \mathbf{s}^{\mathbf{1}}, \mathbf{4} \mathbf{f}^{7}, 4 f^{6} 5 d^{1}, 4 f^{6} 6 p^{1}, 4 f^{5} 5 d^{1} 6 s^{1}$ & 9030 & 5807352 & 1448460 \\
\hline Sm III & $4 f^{6}, 4 f^{5} 5 d^{1}, 4 f^{5} 6 s^{1}, 4 f^{5} 6 p^{1}$ & 3737 & 1045697 & 985731 \\
\hline Sm IV & $\begin{array}{l}\mathbf{4 f ^ { 5 }}, 4 f^{4} 5 d^{1}, 4 f^{4} 6 s^{1}, 4 f^{4} 6 p^{1} \\
\mathbf{4 f}^{7} 6 \mathbf{s}^{2}, 4 f^{7} 5 d^{1} 6 s^{1}, 4 f^{7} 6 s^{1} 6 p^{1}, 4 f^{6} 5 d^{1} 6 s^{2}, 4 f^{7} 5 d^{1} 6 p^{1}\end{array}$ & 1994 & 320633 & 320091 \\
\hline $\mathrm{Eu} \mathrm{I}$ & $\begin{array}{l}4 f^{7} 6 s^{1} 7 s^{1}, 4 f^{6} 5 d^{2} 6 s^{1}, 4 f^{7} 5 d^{2}, 4 f^{7} 6 s^{1} 7 p^{1}, 4 f^{7} 6 s^{1} 6 d^{1}, \\
4 f^{7} 6 s^{1} 8 s^{1}, 4 f^{7} 6 s^{1} 5 f^{1}, 4 f^{7} 6 s^{1} 8 p^{1}, 4 f^{7} 6 s^{1} 7 d^{1}, 4 f^{7} 6 p^{2}\end{array}$ & 103229 & 736608820 & 4101 \\
\hline $\mathrm{Eu} \mathrm{II}$ & $\mathbf{4 f}^{7} \mathbf{6} \mathbf{s}^{1}, 4 f^{7} 5 d^{1}, 4 f^{7} 6 p^{1}, 4 f^{6} 5 d^{1} 6 s^{1}, 4 f^{6} 5 d^{2}$ & 22973 & 21396542 & 910949 \\
\hline Eu III & $4 \mathbf{f}^{7}, 4 f^{6} 5 d^{1}, 4 f^{6} 6 s^{1}, 4 f^{6} 6 p^{1}$ & 5323 & 2073702 & 1651778 \\
\hline Eu IV & $4 \mathbf{f}^{6}, 4 f^{5} 5 d^{1}, 4 f^{5} 6 s^{1}, 4 f^{5} 6 p^{1}$ & 3737 & 1045697 & 1044962 \\
\hline Gd I & 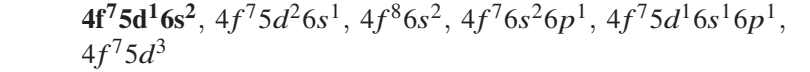 & 103013 & 703084537 & 31461 \\
\hline Gd II & 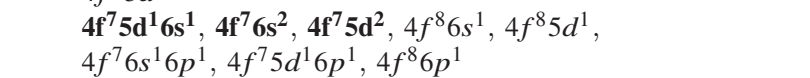 & 46733 & 158102968 & 4161867 \\
\hline Gd III & $4 \mathbf{f}^{8}, 4 f^{7} 5 d^{1}, 4 f^{7} 6 s^{1}, 4 f^{7} 6 p^{1}, 4 f^{7} 7 s^{1}$ & 6637 & 2999281 & 1565172 \\
\hline Gd IV & $4 \mathbf{f}^{7}, 4 f^{6} 5 d^{1}, 4 f^{6} 6 s^{1}, 4 f^{6} 6 p^{1}$ & 5323 & 2073702 & 2012135 \\
\hline $\mathrm{Tb} \mathrm{I}$ & $\begin{array}{l}\mathbf{4 \mathbf { f } ^ { 9 }} \mathbf{6} \mathbf{s}^{2}, 4 f^{8} 5 d^{1} 6 s^{2}, 4 f^{8} 5 d^{2} 6 s^{1}, 4 f^{8} 6 s^{2} 6 p^{1}, 4 f^{9} 6 s^{1} 6 p^{1} \\
4 f^{8} 5 d^{1} 6 s^{1} 6 p^{1}, 4 f^{9} 5 d^{1} 6 s^{1}\end{array}$ & 65817 & 311326160 & 492498 \\
\hline $\mathrm{Tb}$ II & $\mathbf{4 f}^{\mathbf{9}} \mathbf{6} \mathbf{s}^{\mathbf{1}}, 4 f^{8} 5 d^{1} 6 s^{1}, 4 f^{8} 6 s^{2}, 4 f^{8} 5 d^{2}, 4 f^{9} 5 d^{1}$ & 19854 & 11978694 & 3279198 \\
\hline $\mathrm{Tb}$ III & $4 \mathbf{f}^{9}, 4 f^{8} 5 d^{1}, 4 f^{8} 6 s^{1}, 4 f^{8} 6 p^{1}$ & 5194 & 1943961 & 1516250 \\
\hline Tb IV & $4 \mathbf{f}^{8}, 4 f^{7} 5 d^{1}, 4 f^{7} 6 s^{1}, 4 f^{7} 6 p^{1}$ & 5983 & 2545975 & 2330682 \\
\hline Dy I & $\begin{array}{l}4 \mathbf{f}^{10} \mathbf{6 s}^{2}, 4 f^{9} 5 d^{1} 6 s^{2}, 4 f^{10} 6 s^{1} 6 p^{1}, 4 f^{10} 5 d^{1} 6 s^{1}, 4 f^{9} 5 d^{2} 6 s^{1} \\
4 f^{9} 6 s^{2} 6 p^{1}, 4 f^{9} 5 d^{1} 6 s^{1} 6 p^{1}, 4 f^{10} 6 s^{1} 7 s^{1}\end{array}$ & 44669 & 145465351 & 19386 \\
\hline Dy II & $\begin{array}{l}\mathbf{4 f}^{\mathbf{1 0}} \mathbf{6} \mathbf{s}^{\mathbf{1}}, \mathbf{4} \mathbf{f}^{\mathbf{1 0}} \mathbf{5} \mathbf{d}^{\mathbf{1}}, 4 f^{9} 5 d^{1} 6 s^{1}, 4 f^{9} 6 s^{2}, 4 f^{9} 5 d^{2} \\
4 f^{10} 6 p^{1}, 4 f^{9} 6 s^{1} 6 p^{1}\end{array}$ & 16034 & 13700193 & 2227018 \\
\hline Dy III & $4 \mathbf{f}^{10}, 4 f^{9} 5 d^{1}, 4 f^{9} 6 s^{1}, 4 f^{9} 6 p^{1}$ & 3549 & 915339 & 829418 \\
\hline Dy IV & $4 \mathbf{f}^{\mathbf{9}}, 4 f^{8} 5 d^{1}, 4 f^{8} 6 s^{1}, 4 f^{8} 6 p^{1}$ & 5194 & 1943961 & 1901856 \\
\hline Ho I & $\begin{array}{l}\mathbf{4 f ^ { 1 1 }} \mathbf{6 \mathbf { s } ^ { 2 }}, 4 f^{10} 5 d^{1} 6 s^{2}, 4 f^{11} 6 s^{1} 6 p^{1}, 4 f^{10} 6 s^{2} 6 p^{1}, 4 f^{11} 5 d^{1} 6 s^{1} \\
4 f^{10} 5 d^{2} 6 s^{1}, 4 f^{10} 5 d^{1} 6 s^{1} 6 p^{1}, 4 f^{11} 6 s^{1} 7 s^{1}, 4 f^{11} 6 s^{1} 7 p^{1}\end{array}$ & 23182 & 41659671 & 16219 \\
\hline Ho II & $\begin{array}{l}\mathbf{4 f}^{11} \mathbf{6} \mathbf{s}^{\mathbf{1}}, \mathbf{4} \mathbf{f}^{11} \mathbf{5 \mathbf { d } ^ { 1 }}, 4 f^{11} 6 p^{1}, 4 f^{10} 6 s^{1} 6 p^{1}, 4 f^{10} 6 s^{1} 5 d^{1} \\
4 f^{10} 5 d^{1} 6 p^{1}\end{array}$ & 9640 & 5254717 & 510917 \\
\hline Ho III & $4 \mathbf{f}^{\mathbf{1 1}}, 4 f^{10} 5 d^{1}, 4 f^{10} 6 s^{1}, 4 f^{10} 6 p^{1}$ & 1837 & 259812 & 239785 \\
\hline Ho IV & $4 \mathbf{f}^{\mathbf{1 0}}, 4 f^{9} 5 d^{1}, 4 f^{9} 6 s^{1}, 4 f^{9} 6 p^{1}$ & 3549 & 915339 & 897163 \\
\hline
\end{tabular}


Table B4 - continued Summary of HULLAC calculations. The last column shows the number of transitions whose upper level is below the ionization potential.

\begin{tabular}{|c|c|c|c|c|}
\hline Ion & Configurations & $N_{\text {level }}$ & $N_{\text {line }}$ & $N_{\text {line }}^{*}$ \\
\hline Er I & 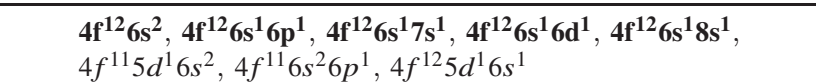 & 1303 & 149737 & 11731 \\
\hline Er II & $\begin{array}{l}6 s^{1} 4 f^{12}, 4 f^{12} 6 p^{1}, 4 f^{12} 5 d^{1}, 4 f^{11} 6 s^{2}, 4 f^{11} 5 d^{1} 6 s^{1} \\
4 f^{11} 5 d^{2}, 4 f^{11} 6 s^{1} 6 p^{1}, 4 f^{11} 5 d^{1} 6 p^{1}\end{array}$ & 5333 & 1620729 & 828267 \\
\hline Er III & $4 f^{12}, 4 f^{11} 5 d^{1}, 4 f^{11} 6 s^{1}, 4 f^{11} 6 p^{1}$ & 723 & 42671 & 40824 \\
\hline Er IV & $\begin{array}{l}\mathbf{4} \mathbf{f}^{\mathbf{1 1}}, 4 f^{10} 6 s^{1}, 4 f^{10} 6 p^{1}, 4 f^{10} 5 d^{1} \\
\mathbf{4 f}^{\mathbf{1 3}} \mathbf{6} \mathbf{s}^{\mathbf{2}}, \mathbf{4} \mathbf{f}^{\mathbf{1 3}} \mathbf{6} \mathbf{s}^{\mathbf{1}} \mathbf{6} \mathbf{p}^{\mathbf{1}}, \mathbf{4} \mathbf{f}^{\mathbf{1 3}} \mathbf{5} \mathbf{d}^{\mathbf{1}} \mathbf{6} \mathbf{s}^{\mathbf{1}}, \mathbf{4} \mathbf{f}^{\mathbf{1 3}} \mathbf{6} \mathbf{s}^{\mathbf{1}} 7 \mathbf{s}^{\mathbf{1}}, \mathbf{4 f}^{\mathbf{1 3}} \mathbf{6} \mathbf{s}^{\mathbf{1}} \mathbf{8} \mathbf{s}^{\mathbf{1}},\end{array}$ & 1837 & 259812 & 257750 \\
\hline Tm I & $\begin{array}{l}\mathbf{4 f}^{\mathbf{1 2}} \mathbf{5} \mathbf{d}^{\mathbf{1}} \mathbf{6} \mathbf{s}^{\mathbf{2}}, \mathbf{4}^{\mathbf{1 2}} \mathbf{6} \mathbf{s}^{\mathbf{2}} \mathbf{6} \mathbf{p}^{\mathbf{1}}, \mathbf{4 f}^{\mathbf{1 3}} \mathbf{6} \mathbf{s}^{1} \mathbf{p}^{\mathbf{1}}, \mathbf{4 f}^{\mathbf{1 3}} \mathbf{5} \mathbf{d}^{1} \mathbf{6} \mathbf{p}^{1}, \mathbf{4 f}^{\mathbf{1 3}} \mathbf{6} \mathbf{s}^{1} \mathbf{6} \mathbf{d}^{\mathbf{1}} \\
4 f^{12} 5 d^{1} 6 s^{1} 6 p^{1}, 4 f^{13} 6 p^{2}, 4 f^{13} 6 s^{1} 8 p^{1}\end{array}$ & 1716 & 172582 & 25853 \\
\hline Tm II & $\begin{array}{l}4 f^{13} 6 s^{1}, 4 f^{12} 6 s^{2}, 4 f^{13} 5 d^{1}, 4 f^{13} 6 p^{1}, 4 f^{12} 5 d^{1} 6 s^{1} \\
4 f^{12} 5 d^{2}, 4 f^{12} 6 s^{1} 6 p^{1}, 4 f^{12} 5 d^{1} 6 p^{1}\end{array}$ & 1484 & 205258 & 158892 \\
\hline Tm III & $\begin{array}{l}4 \mathbf{f}^{\mathbf{1 3}}, 4 f^{12} 5 d^{1}, 4 f^{12} 6 s^{1}, 4 f^{12} 6 p^{1}, 4 f^{11} 5 d^{1} 6 s^{1} \\
4 f^{11} 5 d^{1} 6 p^{1}, 4 f^{11} 6 s^{1} 6 p^{1}\end{array}$ & 3666 & 824686 & 5582 \\
\hline Tm IV & $\begin{array}{l}\mathbf{4 f ^ { 1 2 }}, 4 f^{11} 5 d^{1}, 4 f^{11} 6 s^{1}, 4 f^{11} 6 p^{1} \\
\mathbf{4 f}^{\mathbf{1 4}} \mathbf{6 s}^{\mathbf{2}}, \mathbf{4 f}^{\mathbf{1 4}} \mathbf{6} \mathbf{s}^{\mathbf{1}} \mathbf{p}^{\mathbf{1}}, \mathbf{4 f}^{\mathbf{1 4}} \mathbf{6} \mathbf{s}^{\mathbf{1}} \mathbf{5} \mathbf{d}^{\mathbf{1}}, \mathbf{4} \mathbf{f}^{\mathbf{1 4}} \mathbf{6 s}^{\mathbf{1}} \mathbf{7} \mathbf{s}^{\mathbf{1}}, \mathbf{4 f}^{\mathbf{1 4}} \mathbf{6 s}^{\mathbf{1}} \mathbf{6} \mathbf{d}^{\mathbf{1}}\end{array}$ & 723 & 42671 & 42671 \\
\hline $\mathrm{Yb} \mathrm{I}$ & 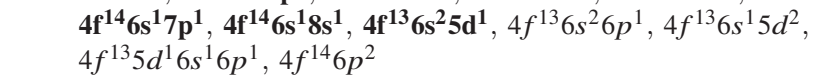 & 446 & 20948 & 2821 \\
\hline $\mathrm{Yb}$ II & 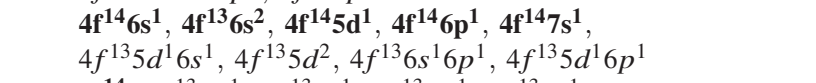 & 265 & 8109 & 8024 \\
\hline Yb III & $\begin{array}{l}4 f^{14}, 4 f^{13} 5 d^{1}, 4 f^{13} 6 s^{1}, 4 f^{13} 6 p^{1}, 4 f^{13} 7 s^{1} \\
4 f^{13} 6 d^{1}, 4 f^{12} 5 d^{1} 6 s^{1}, 4 f^{12} 5 d^{1} 6 p^{1}, 4 f^{12} 6 s^{1} 6 p^{1}\end{array}$ & 1039 & 71310 & 616 \\
\hline Yb IV & $\begin{array}{l}\mathbf{4 f ^ { 1 3 }}, 4 f^{12} 5 d^{1}, 4 f^{12} 6 s^{1}, 4 f^{12} 6 p^{1} \\
\mathbf{5 d}^{1} \mathbf{6} \mathbf{s}^{2}, 6 s^{2} 6 p^{1}, 5 d^{1} 6 s^{1} 6 p^{1}, 5 d^{2} 6 s^{1}, 6 s^{2} 7 s^{1}\end{array}$ & 202 & 3797 & 3797 \\
\hline $\mathrm{Lu} \mathrm{I}$ & $\begin{array}{l}6 s^{2} 6 d^{1}, 6 s^{2} 8 s^{1}, 6 s^{2} 7 p^{1}, 6 s^{1} 6 p^{2}, 6 s^{2} 5 f^{1} \\
6 s^{2} 7 d^{1}\end{array}$ & 61 & 602 & 391 \\
\hline Lu II & $\begin{array}{l}4 \mathbf{f}^{\mathbf{1 4}} \mathbf{6 \mathbf { s } ^ { 2 }}, 4 f^{14} 5 d^{1} 6 s^{1}, 4 f^{14} 6 s^{1} 6 p^{1}, 4 f^{14} 5 d^{2}, 4 f^{14} 5 d^{1} 6 p^{1}, \\
4 f^{14} 6 s^{1} 7 s^{1}, 4 f^{14} 6 s^{1} 6 d^{1}, 4 f^{14} 5 d^{1} 7 s^{1}, 4 f^{14} 5 d^{1} 6 d^{1}\end{array}$ & 58 & 400 & 335 \\
\hline Lu III & $\begin{array}{l}\mathbf{4 f ^ { 1 4 }} \mathbf{6} \mathbf{s}^{1}, 4 f^{14} 5 d^{1}, 4 f^{14} 6 p^{1}, 4 f^{14} 7 s^{1}, 4 f^{14} 6 d^{1} \\
4 f^{13} 5 d^{1} 6 s^{1}, 4 f^{13} 5 d^{1} 6 p^{1}, 4 f^{13} 6 s^{1} 6 p^{1}\end{array}$ & 184 & 2784 & 1603 \\
\hline Lu IV & $\begin{array}{l}\mathbf{4 \mathbf { f } ^ { 1 4 }}, 4 f^{13} 5 d^{1}, 4 f^{13} 6 s^{1}, 4 f^{13} 6 p^{1}, 4 f^{13} 6 d^{1} \\
4 f^{13} 7 s^{1}\end{array}$ & 61 & 338 & 338 \\
\hline Hf I & $\begin{array}{l}\mathbf{5 d}^{2} 6 \mathbf{s}^{2}, 5 d^{2} 6 s^{1} 6 p^{1}, 5 d^{3} 6 s^{1}, 5 d^{4}, 5 d^{3} 6 p^{1} \\
5 d^{2} 6 s^{1} 7 s^{1}\end{array}$ & 313 & 11138 & 4146 \\
\hline Hf II & $\begin{array}{l}\mathbf{5 d}^{1} \mathbf{6} \mathbf{s}^{2}, 5 d^{2} 6 s^{1}, 5 d^{3}, 5 d^{1} 6 s^{1} 6 p^{1}, 5 d^{2} 6 p^{1} \\
5 d^{2} 7 s^{1}, 5 d^{1} 6 s^{1} 7 s^{1}\end{array}$ & 129 & 2418 & 2418 \\
\hline Hf III & $\begin{array}{l}5 \mathbf{d}^{2}, 5 d^{1} 6 s^{1}, 6 s^{2}, 5 d^{1} 6 p^{1}, 6 s^{1} 6 p^{1} \\
5 d^{1} 6 d^{1}, 5 d^{1} 7 s^{1}, 5 d^{1} 7 p^{1}\end{array}$ & 64 & 594 & 594 \\
\hline Hf IV & $\begin{array}{l}\mathbf{4 f}^{\mathbf{1 4}} \mathbf{5 \mathbf { d } ^ { 1 }}, 4 f^{14} 6 s^{1}, 4 f^{14} 6 p^{1}, 4 f^{14} 6 d^{1}, 4 f^{14} 7 s^{1} \\
4 f^{14} 5 f^{1}, 4 f^{14} 7 p^{1}, 4 f^{14} 7 d^{1}\end{array}$ & 14 & 35 & 35 \\
\hline Ta I & $\begin{array}{l}\mathbf{5 d}^{\mathbf{3}} \mathbf{6} \mathbf{s}^{\mathbf{2}}, \mathbf{5 \mathbf { d } ^ { 5 }}, 5 d^{4} 6 s^{1}, 5 d^{3} 6 s^{1} 6 p^{1}, 5 d^{2} 6 s^{2} 6 p^{1} \\
5 d^{4} 6 p^{1}, 5 d^{3} 6 s^{1} 7 s^{1}, 5 d^{3} 6 s^{1} 8 s^{1}\end{array}$ & 705 & 58122 & 25703 \\
\hline Ta II & $\begin{array}{l}\mathbf{5 d}^{3} \mathbf{6} \mathbf{s}^{\mathbf{1}}, \mathbf{5} \mathbf{d}^{\mathbf{2}} \mathbf{6} \mathbf{s}^{2}, \mathbf{5} \mathbf{d}^{4}, 5 d^{3} 6 p^{1}, 5 d^{2} 6 s^{1} 6 p^{1} \\
5 d^{3} 7 s^{1}, 5 d^{3} 6 d^{1}\end{array}$ & 487 & 27199 & 27162 \\
\hline Ta III & $\begin{array}{l}\mathbf{5} \mathbf{d}^{3}, 5 d^{2} 6 s^{1}, 5 d^{2} 6 p^{1}, 5 d^{1} 6 s^{2}, 5 d^{1} 6 s^{1} 6 p^{1} \\
5 d^{2} 6 d^{1}, 5 d^{2} 7 s^{1}\end{array}$ & 188 & 4452 & 4452 \\
\hline Ta IV & $\begin{array}{l}\mathbf{5 d}^{2}, 5 d^{1} 6 s^{1}, 5 d^{1} 6 p^{1}, 6 s^{2}, 6 s^{1} 6 p^{1} \\
5 d^{1} 6 d^{1}, 5 d^{1} 7 s^{1}\end{array}$ & 52 & 336 & 336 \\
\hline
\end{tabular}


Table B5. Summary of HULLAC calculations. The last column shows the number of transitions whose upper level is below the ionization potential.

\begin{tabular}{|c|c|c|c|c|}
\hline Ion & Configurations & $N_{\text {level }}$ & $N_{\text {line }}$ & $N_{\text {line }}^{*}$ \\
\hline W I & 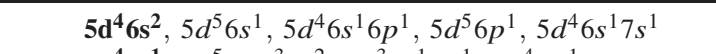 & 808 & 61598 & 22278 \\
\hline W II & $\begin{array}{l}\mathbf{5 d}^{4} \mathbf{6} \mathbf{s}^{1}, 5 d^{5}, 5 d^{3} 6 s^{2}, 5 d^{3} 6 s^{1} 6 p^{1}, 5 d^{4} 6 p^{1} \\
5 d^{4} 7 s^{1}, 5 d^{4} 6 d^{1}\end{array}$ & 851 & 84312 & 80301 \\
\hline W III & $\begin{array}{l}\mathbf{5 d}^{4}, 5 d^{3} 6 s^{1}, 5 d^{2} 6 s^{2}, 5 d^{3} 6 p^{1}, 5 d^{2} 6 s^{1} 6 p^{1} \\
5 d^{3} 7 s^{1}, 5 d^{3} 6 d^{1}\end{array}$ & 487 & 27199 & 27199 \\
\hline W IV & $\begin{array}{l}\mathbf{5 d}^{3}, 5 d^{2} 6 s^{1}, 5 d^{2} 6 p^{1}, 5 d^{1} 6 s^{2}, 5 d^{1} 6 s^{1} 6 p^{1} \\
5 d^{2} 7 s^{1}, 5 d^{2} 6 d^{1}\end{array}$ & 188 & 4452 & 4452 \\
\hline Re I & $\begin{array}{l}\mathbf{5 d}^{\mathbf{5}} \mathbf{6} \mathbf{s}^{\mathbf{2}}, \mathbf{5} \mathbf{d}^{\mathbf{6}} \mathbf{6} \mathbf{s}^{1}, 5 d^{5} 6 s^{1} 6 p^{1}, 5 d^{4} 6 s^{2} 6 p^{1}, 5 d^{5} 6 s^{1} 7 s^{1} \\
5 d^{6} 6 p^{1}, 5 d^{5} 6 s^{1} 6 d^{1}, 5 d^{5} 6 s^{1} 8 s^{1}, 5 d^{4} 6 s^{2} 7 s^{1}\end{array}$ & 1875 & 389214 & 18721 \\
\hline Re II & $\begin{array}{l}\mathbf{5 d}^{\mathbf{5}} \mathbf{6} \mathbf{s}^{1}, \mathbf{5 d}^{\mathbf{4}} \mathbf{6} \mathbf{s}^{2}, 5 d^{5} 6 p^{1}, 5 d^{6}, 5 d^{4} 6 s^{1} 6 p^{1} \\
5 d^{5} 7 s^{1}, 5 d^{5} 6 d^{1}\end{array}$ & 1122 & 138447 & 102286 \\
\hline Re III & $\begin{array}{l}\mathbf{5 d}^{5}, 5 d^{4} 6 s^{1}, 5 d^{4} 6 p^{1}, 5 d^{3} 6 s^{1} 6 p^{1}, 5 d^{3} 6 s^{2} \\
5 d^{4} 7 s^{1}, 5 d^{4} 6 d^{1}\end{array}$ & 851 & 84312 & 83853 \\
\hline Re IV & $\begin{array}{l}\mathbf{5 d}^{4}, 5 d^{3} 6 s^{1}, 5 d^{3} 6 p^{1}, 5 d^{2} 6 s^{2}, 5 d^{2} 6 s^{1} 6 p^{1} \\
5 d^{3} 7 s^{1}, 5 d^{3} 6 d^{1}\end{array}$ & 487 & 27199 & 27199 \\
\hline Os I & $\begin{array}{l}\mathbf{5 d}^{6} 6 \mathbf{s}^{2}, 5 d^{7} 6 s^{1}, 5 d^{6} 6 s^{1} 6 p^{1}, 5 d^{6} 6 s^{1} 7 s^{1}, 5 d^{7} 6 p^{1} \\
5 d^{7} 7 s^{1}, 5 d^{7} 7 p^{1}, 5 d^{7} 6 d^{1}\end{array}$ & 984 & 107910 & 27186 \\
\hline Os II & $\begin{array}{l}\mathbf{5 d}^{6} \mathbf{6} \mathbf{s}^{1}, 5 d^{6} 6 p^{1}, 5 d^{7}, 5 d^{6} 6 p^{1}, 5 d^{5} 6 s^{1} 6 p^{1} \\
5 d^{6} 7 s^{1}, 5 d^{6} 6 d^{1}, 5 d^{5} 6 s^{1} 7 p^{1}\end{array}$ & 1435 & 195855 & 112108 \\
\hline Os III & $\begin{array}{l}\mathbf{5 d}^{6}, 5 d^{5} 6 s^{1}, 5 d^{5} 6 p^{1}, 5 d^{4} 6 s^{1} 6 p^{1}, 5 d^{5} 6 d^{1} \\
5 d^{5} 7 s^{1}\end{array}$ & 1088 & 130730 & 117655 \\
\hline Os IV & $\begin{array}{l}\mathbf{5 d}^{5}, 5 d^{4} 6 s^{1}, 5 d^{4} 6 p^{1}, 5 d^{3} 6 s^{2}, 5 d^{3} 6 s^{1} 6 p^{1} \\
5 d^{4} 7 s^{1}, 5 d^{4} 6 d^{1}\end{array}$ & 851 & 84312 & 84312 \\
\hline Ir I & $\begin{array}{l}\mathbf{5 d}^{7} \mathbf{6} \mathbf{s}^{2}, \mathbf{5 d}^{9}, 5 d^{8} 6 s^{1}, 5 d^{7} 6 s^{1} 6 p^{1}, 5 d^{7} 6 s^{1} 7 s^{1} \\
5 d^{8} 6 p^{1}, 5 d^{8} 7 s^{1}\end{array}$ & 385 & 16855 & 9449 \\
\hline Ir II & $\begin{array}{l}\mathbf{5 d}^{7} \mathbf{6} \mathbf{s}^{1}, 5 d^{8}, 5 d^{6} 6 s^{2}, 5 d^{7} 6 p^{1}, 5 d^{6} 6 s^{1} 6 p^{1} \\
5 d^{7} 7 s^{1}, 5 d^{7} 7 p^{1}\end{array}$ & 699 & 32647 & 29639 \\
\hline Ir III & $\begin{array}{l}5 \mathbf{d}^{7}, 5 d^{6} 6 s^{1}, 5 d^{6} 6 p^{1}, 5 d^{5} 6 s^{2}, 5 d^{6} 6 d^{1} \\
5 d^{6} 7 s^{1}, 5 d^{6} 7 p^{1}\end{array}$ & 818 & 76592 & 73814 \\
\hline Ir IV & $\begin{array}{l}\mathbf{5 d}^{6}, 5 d^{5} 6 s^{1}, 5 d^{5} 6 p^{1}, 5 d^{4} 6 s^{2}, 5 d^{5} 6 d^{1} \\
5 d^{5} 7 s^{1}, 5 d^{5} 7 p^{1}\end{array}$ & 976 & 104622 & 104622 \\
\hline Pt I & $\begin{array}{l}\mathbf{5 d ^ { 9 }} \mathbf{6 s}^{\mathbf{1}}, \mathbf{5 d}^{\mathbf{1 0}}, \mathbf{5 d}^{\mathbf{9}} \mathbf{6} \mathbf{p}^{\mathbf{1}}, \mathbf{5 d}^{\mathbf{9}} \mathbf{7} \mathbf{s}^{\mathbf{1}}, 5 d^{8} 6 s^{2} \\
5 d^{8} 6 s^{1} 6 p^{1}, 5 d^{8} 6 s^{1} 7 s^{1}\end{array}$ & 152 & 2729 & 1637 \\
\hline Pt II & $\begin{array}{l}5 \mathbf{d}^{9}, 5 d^{8} 6 s^{1}, 5 d^{7} 6 s^{2}, 5 d^{8} 6 p^{1}, 5 d^{8} 7 s^{1} \\
5 d^{8} 6 d^{1}, 5 d^{8} 8 s^{1}, 5 d^{8} 7 d^{1}\end{array}$ & 248 & 4837 & 4759 \\
\hline Pt III & $\mathbf{5 d}^{8}, 5 d^{7} 6 s^{1}, 5 d^{7} 6 p^{1}, 5 d^{7} 7 s^{1}, 5 d^{6} 6 s^{1} 6 p^{1}$ & 555 & 19157 & 18477 \\
\hline Pt IV & $\begin{array}{l}\mathbf{5} \mathbf{d}^{7}, 5 d^{6} 6 s^{1}, 5 d^{6} 6 p^{1}, 5 d^{6} 6 d^{1}, 5 d^{6} 7 s^{1} \\
5 d^{6} 7 p^{1}\end{array}$ & 781 & 70102 & 70102 \\
\hline $\mathrm{Au} \mathrm{I}$ & $\begin{array}{l}\mathbf{5 d}^{10} 6 \mathbf{s}^{1}, 5 d^{9} 6 s^{2}, 5 d^{10} 6 p^{1}, 5 d^{9} 6 s^{1} 6 p^{1}, 5 d^{10} 7 s^{1} \\
5 d^{10} 7 p^{1}, 5 d^{10} 6 d^{1}, 5 d^{10} 8 s^{1}, 5 d^{10} 8 p^{1}\end{array}$ & 36 & 141 & 101 \\
\hline $\mathrm{Au} \mathrm{II}$ & $\begin{array}{l}\mathbf{5 d}^{10}, 5 d^{9} 6 s^{1}, 5 d^{8} 6 s^{2}, 5 d^{9} 6 p^{1}, 5 d^{9} 7 s^{1} \\
5 d^{9} 6 d^{1}, 5 d^{9} 7 p^{1}\end{array}$ & 60 & 516 & 516 \\
\hline $\mathrm{Au}$ III & $\begin{array}{l}5 \mathbf{d}^{9}, 5 d^{8} 6 s^{1}, 5 d^{8} 6 p^{1}, 5 d^{7} 6 s^{2}, 5 d^{8} 7 s^{1} \\
5 d^{8} 6 d^{1}, 5 d^{8} 7 p^{1}\end{array}$ & 210 & 5764 & 5690 \\
\hline $\mathrm{Au} \mathrm{IV}$ & $\begin{array}{l}5 \mathbf{d}^{8}, 5 d^{7} 6 s^{1}, 5 d^{7} 6 p^{1}, 5 d^{6} 6 s^{2}, 5 d^{7} 7 s^{1} \\
5 d^{7} 6 d^{1}, 5 d^{7} 7 p^{1}\end{array}$ & 507 & 29606 & 29606 \\
\hline
\end{tabular}


Table B5 - continued Summary of HULLAC calculations. The last column shows the number of transitions whose upper level is below the ionization potential.

\begin{tabular}{|c|c|c|c|c|}
\hline Ion & Configurations & $N_{\text {level }}$ & $N_{\text {line }}$ & $N_{\text {line }}^{*}$ \\
\hline $\mathrm{Hg} \mathrm{I}$ & $\begin{array}{l}\mathbf{5 d}^{10} \mathbf{6 s}^{2}, 5 d^{10} 6 s^{1} 6 p^{1}, 5 d^{10} 6 s^{1} 7 s^{1}, 5 d^{9} 6 s^{2} 6 p^{1}, 5 d^{10} 6 s^{1} 7 p^{1} \\
5 d^{10} 6 s^{1} 6 d^{1}, 5 d^{10} 6 s^{1} 8 s^{1}, 5 d^{10} 6 s^{1} 8 p^{1}, 5 d^{10} 6 s^{1} 7 d^{1}, 5 d^{10} 6 s^{1} 5 f^{1}\end{array}$ & 41 & 229 & 177 \\
\hline Hg II & $\begin{array}{l}\mathbf{5 d}^{10} \mathbf{6} \mathbf{s}^{1}, 5 d^{9} 6 s^{2}, 5 d^{10} 6 p^{1}, 5 d^{9} 6 s^{1} 6 p^{1}, 5 d^{10} 7 s^{1} \\
5 d^{10} 6 d^{1}, 5 d^{10} 7 p^{1}, 5 d^{10} 8 s^{1}, 5 d^{10} 5 f^{1}, 5 d^{10} 7 d^{1}\end{array}$ & 38 & 180 & 180 \\
\hline Hg III & $\begin{array}{l}\mathbf{5} \mathbf{d}^{10}, 5 d^{9} 6 s^{1}, 5 d^{8} 6 s^{2}, 5 d^{9} 6 p^{1}, 5 d^{8} 6 s^{1} 6 p^{1} \\
5 d^{9} 7 s^{1}, 5 d^{9} 6 d^{1}\end{array}$ & 138 & 1948 & 1948 \\
\hline $\mathrm{Hg}$ IV & $\begin{array}{l}\mathbf{5 d}^{9}, 5 d^{8} 6 s^{1}, 5 d^{8} 6 p^{1}, 5 d^{7} 6 s^{2}, 5 d^{8} 6 d^{1} \\
5 d^{8} 7 s^{1}\end{array}$ & 165 & 2882 & 2882 \\
\hline Tl I & $\begin{array}{l}\mathbf{6 s}^{\mathbf{2}} \mathbf{6} \mathbf{p}^{\mathbf{1}}, \mathbf{6 s}^{\mathbf{2}} \mathbf{7} \mathbf{s}^{\mathbf{1}}, \mathbf{6 s}^{\mathbf{2}} \mathbf{7} \mathbf{p}^{\mathbf{1}}, 6 s^{2} 6 d^{1}, 6 s^{2} 8 s^{1}, \\
6 s^{2} 8 p^{1}, 6 s^{2} 7 d^{1} \\
\mathbf{5} \mathbf{d}^{\mathbf{1 0}} \mathbf{6} \mathbf{s}^{\mathbf{2}}, 5 d^{10} 6 s^{1} 6 p^{1}, 5 d^{10} 6 s^{1} 7 s^{1}, 5 d^{9} 6 s^{2} 6 p^{1}, 5 d^{10} 6 s^{1} 6 d^{1} .\end{array}$ & 12 & 30 & 30 \\
\hline Tl II & $\begin{array}{l}5 d^{10} 6 p^{2}, 5 d^{10} 6 s^{1} 7 p^{1}, 5 d^{10} 6 s^{1} 8 s^{1}, 5 d^{10} 6 s^{1} 5 f^{1}, 5 d^{10} 6 s^{1} 7 d^{1} \\
5 d^{10} 6 s^{1} 8 p^{1} \\
\mathbf{5 d}^{\mathbf{1 0}} \mathbf{6} \mathbf{s}^{1}, 5 d^{10} 6 p^{1}, 5 d^{9} 6 s^{2}, 5 d^{9} 6 s^{1} 6 p^{1}, 5 d^{10} 7 s^{1}\end{array}$ & 46 & 312 & 312 \\
\hline Tl III & $\begin{array}{l}5 d^{10} 6 d^{1}, 5 d^{10} 7 p^{1}, 5 d^{10} 5 f^{1}, 5 d^{10} 8 s^{1}, 5 d^{10} 7 d^{1} \\
5 d^{10} 8 p^{1}\end{array}$ & 40 & 195 & 195 \\
\hline Tl IV & $\begin{array}{l}\mathbf{5 d ^ { 1 0 }}, 5 d^{9} 6 s^{1}, 5 d^{9} 6 p^{1}, 5 d^{9} 6 d^{1}, 5 d^{9} 7 s^{1} \\
5 d^{9} 8 s^{1}\end{array}$ & 43 & 234 & 234 \\
\hline $\mathrm{Pb} \mathrm{I}$ & $\begin{array}{l}\mathbf{6 s}^{\mathbf{2}} \mathbf{6} \mathbf{p}^{\mathbf{2}}, \mathbf{6 s}^{\mathbf{2}} \mathbf{6} \mathbf{p}^{\mathbf{1}} 7 \mathbf{s}^{\mathbf{1}}, \mathbf{6 s}^{\mathbf{2}} \mathbf{6} \mathbf{p}^{\mathbf{1}} \mathbf{7} \mathbf{p}^{\mathbf{1}}, \mathbf{6 s}^{\mathbf{2}} \mathbf{6} \mathbf{p}^{\mathbf{1}} \mathbf{6} \mathbf{d}^{\mathbf{1}}, \mathbf{6 s}^{\mathbf{2}} \mathbf{6} \mathbf{p}^{\mathbf{1}} \mathbf{8} \mathbf{s}^{\mathbf{1}} \\
\mathbf{6 s}^{\mathbf{2}} \mathbf{6} \mathbf{p}^{\mathbf{1}} \mathbf{8} \mathbf{p}^{\mathbf{1}}, 6 s^{2} 6 p^{1} 7 d^{1}, 6 s^{2} 6 p^{1} 5 f^{1}, 6 s^{2} 6 p^{1} 9 s^{1}, 6 s^{2} 6 p^{1} 9 p^{1}, \\
6 s^{2} 6 p^{1} 8 d^{1} \\
\mathbf{6 s}^{\mathbf{2}} \mathbf{6} \mathbf{p}^{\mathbf{1}}, 6 s^{1} 6 p^{2}, 6 s^{2} 7 s^{1}, 6 s^{2} 6 d^{1}, 6 s^{2} 7 p^{1},\end{array}$ & 95 & 1398 & 1182 \\
\hline $\mathrm{Pb}$ II & $\begin{array}{l}6 s^{2} 8 s^{1}, 6 s^{2} 5 f^{1}, 6 s^{2} 7 d^{1}, 6 s^{2} 8 p^{1}, 6 s^{2} 9 s^{1} \\
6 s^{2} 6 f^{1}, 6 s^{2} 8 d^{1} \\
\mathbf{5 d}^{10} 6 \mathbf{s}^{2}, 5 d^{10} 6 s^{1} 6 p^{1}, 5 d^{10} 6 p^{2}, 5 d^{10} 6 s^{1} 7 s^{1}, 5 d^{10} 6 s^{1} 6 d^{1}\end{array}$ & 27 & 119 & 119 \\
\hline $\mathrm{Pb}$ III & $\begin{array}{l}5 d^{9} 6 s^{2} 6 p^{1}, 5 d^{10} 6 s^{1} 7 p^{1}, 5 d^{10} 6 s^{1} 5 f^{1}, 5 d^{10} 6 s^{1} 8 s^{1}, 5 d^{10} 6 s^{1} 7 d^{1} \\
5 d^{10} 6 s^{1} 8 p^{1}, 5 d^{10} 6 s^{1} 6 f^{1} \\
\mathbf{5 d}^{10} 6 \mathbf{s}^{1}, 5 d^{10} 6 p^{1}, 5 d^{9} 6 s^{2}, 5 d^{9} 6 s^{1} 6 p^{1}, 5 d^{10} 6 d^{1}\end{array}$ & 50 & 343 & 343 \\
\hline $\mathrm{Pb} \mathrm{IV}$ & $\begin{array}{l}5 d^{10} 7 s^{1}, 5 d^{10} 7 p^{1}, 5 d^{10} 5 f^{1}, 5 d^{10} 8 s^{1}, 5 d^{10} 7 d^{1} \\
5 d^{9} 6 p^{2}, 5 d^{10} 8 p^{1}\end{array}$ & 68 & 746 & 721 \\
\hline Bi I & $\begin{array}{l}\mathbf{6} \mathbf{p}^{\mathbf{2}} 7 \mathbf{s}^{1}, \mathbf{6} \mathbf{p}^{3}, 6 p^{2} 7 p^{1}, 6 p^{2} 6 d^{1}, 6 p^{2} 8 s^{1} \\
6 p^{2} 8 p^{1}, 6 p^{2} 7 d^{1}, 6 p^{2} 9 s^{1}, 6 p^{2} 9 p^{1}, 6 p^{2} 8 d^{1} \\
\mathbf{6 s}^{\mathbf{2}} \mathbf{6} \mathbf{p}^{\mathbf{2}}, \mathbf{6 s}^{\mathbf{2}} \mathbf{6} \mathbf{p}^{1} 7 \mathbf{s}^{1}, 6 s^{1} 6 p^{3}, 6 s^{2} 6 p^{1} 6 d^{1}, 6 s^{2} 6 p^{1} 7 p^{1}\end{array}$ & 176 & 4986 & 270 \\
\hline Bi II & $\begin{array}{l}6 s^{2} 6 p^{1} 8 s^{1}, 6 s^{2} 6 p^{1} 5 f^{1}, 6 s^{2} 6 p^{1} 7 d^{1}, 6 s^{2} 6 p^{1} 8 p^{1}, 6 s^{2} 6 p^{1} 9 s^{1} \\
6 s^{2} 6 p^{1} 6 f^{1}, 6 s^{2} 6 p^{1} 8 d^{1}\end{array}$ & 107 & 1685 & 744 \\
\hline Bi III & $\begin{array}{l}\mathbf{6 s}^{2} \mathbf{6} \mathbf{p}^{1}, 6 s^{1} 6 p^{2}, 6 s^{2} 7 s^{1}, 6 s^{2} 6 d^{1}, 6 s^{2} 7 p^{1} \\
6 s^{2} 5 f^{1}, 6 s^{2} 8 s^{1}, 6 s^{2} 7 d^{1}, 6 s^{2} 8 p^{1}, 6 s^{2} 6 f^{1}\end{array}$ & 24 & 98 & 98 \\
\hline Bi IV & $\begin{array}{l}\mathbf{5 d}^{10} 6 s^{2}, 5 d^{10} 6 s^{1} 6 p^{1}, 5 d^{10} 6 p^{2}, 5 d^{10} 6 s^{1} 6 d^{1}, 5 d^{10} 6 s^{1} 7 s^{1} \\
5 d^{9} 6 s^{2} 6 p^{1}, 5 d^{10} 6 s^{1} 7 p^{1}, 5 d^{10} 6 s^{1} 5 f^{1}, 5 d^{10} 6 s^{1} 8 s^{1}, 5 d^{10} 6 s^{1} 7 d^{1}\end{array}$ & 42 & 262 & 262 \\
\hline Po I & $\begin{array}{l}\mathbf{6 p}^{\mathbf{4}}, \mathbf{6}^{\mathbf{3}} 7 \mathbf{s}^{1}, \mathbf{6} \mathbf{p}^{\mathbf{3}} \mathbf{7} \mathbf{p}^{\mathbf{1}}, \mathbf{6} \mathbf{p}^{\mathbf{3}} \mathbf{6} \mathbf{d}^{\mathbf{1}}, \mathbf{6 p}^{\mathbf{3}} \mathbf{8} \mathbf{s}^{\mathbf{1}} \\
6 p^{3} 8 p^{1}, 6 p^{3} 7 d^{1}, 6 p^{3} 9 p^{1}, 6 p^{3} 8 d^{1}, 6 p^{3} 10 p^{1}\end{array}$ & 251 & 9553 & 135 \\
\hline Po II & $\begin{array}{l}\mathbf{s s}^{2} \mathbf{6} \mathbf{p}^{3}, 6 s^{1} 6 p^{4}, 6 s^{2} 6 p^{2} 7 s^{1}, 6 s^{2} 6 p^{2} 6 d^{1}, 6 s^{2} 6 p^{2} 7 p^{1} \\
6 s^{2} 6 p^{2} 8 s^{1}, 6 s^{2} 6 p^{2} 7 d^{1}, 6 s^{2} 6 p^{2} 5 f^{1}, 6 s^{2} 6 p^{2} 8 p^{1}, 6 s^{2} 6 p^{2} 9 s^{1}\end{array}$ & 165 & 4238 & 873 \\
\hline Po III & $\begin{array}{l}\mathbf{6 s}^{2} \mathbf{6} \mathbf{p}^{2}, 6 s^{1} 6 p^{3}, 6 s^{2} 6 p^{1} 6 d^{1}, 6 s^{2} 6 p^{1} 7 s^{1}, 6 s^{2} 6 p^{1} 7 p^{1} \\
6 s^{2} 6 p^{1} 7 d^{1}, 6 s^{2} 6 p^{1} 8 s^{1}\end{array}$ & 57 & 419 & 369 \\
\hline Po IV & $\begin{array}{l}\mathbf{6 s}^{2} \mathbf{6} \mathbf{p}^{1}, 6 s^{1} 6 p^{2}, 6 s^{2} 6 d^{1}, 6 s^{2} 7 s^{1}, 6 s^{2} 7 p^{1} \\
6 s^{2} 7 d^{1}, 6 s^{2} 8 s^{1}\end{array}$ & 18 & 48 & 48 \\
\hline At I & $\begin{array}{l}\mathbf{6} \mathbf{p}^{5}, 6 p^{4} 7 s^{1}, 6 p^{4} 7 p^{1}, 6 p^{4} 6 d^{1}, 6 p^{4} 8 s^{1} \\
6 p^{4} 8 p^{1}, 6 p^{4} 7 d^{1} \\
\mathbf{6 s}^{2} \mathbf{6} \mathbf{p}^{4}, 6 s^{2} 6 p^{3} 7 s^{1}, 6 s^{1} 6 p^{5}, 6 s^{2} 6 p^{3} 6 d^{1}, 6 s^{2} 6 p^{3} 7 p^{1}\end{array}$ & 116 & 2136 & 3 \\
\hline At II & $\begin{array}{l}6 s^{2} 6 p^{3} 8 s^{1}, 6 s^{2} 6 p^{3} 7 d^{1}, 6 s^{2} 6 p^{3} 5 f^{1}, 6 s^{2} 6 p^{3} 8 p^{1}, 6 s^{2} 6 p^{3} 9 s^{1} \\
6 s^{2} 6 p^{3} 8 d^{1}, 6 s^{2} 6 p^{3} 6 f^{1}\end{array}$ & 289 & 11510 & 881 \\
\hline At III & $\begin{array}{l}\mathbf{6 s}^{\mathbf{2}} \mathbf{6} \mathbf{p}^{\mathbf{3}}, 6 s^{1} 6 p^{4}, 6 s^{2} 6 p^{2} 6 d^{1}, 6 s^{2} 6 p^{2} 7 s^{1}, 6 s^{2} 6 p^{2} 8 s^{1} \\
6 s^{2} 6 p^{2} 7 d^{1}, 6 s^{2} 6 p^{2} 9 s^{1}, 6 s^{2} 6 p^{2} 8 d^{1}\end{array}$ & 121 & 431 & 317 \\
\hline At IV & $\begin{array}{l}\mathbf{6 s}^{2} \mathbf{6} \mathbf{p}^{2}, 6 s^{1} 6 p^{3}, 6 s^{2} 6 p^{1} 6 d^{1}, 6 s^{2} 6 p^{1} 7 s^{1}, 6 s^{2} 6 p^{1} 7 d^{1} \\
6 s^{2} 6 p^{1} 8 s^{1}, 6 s^{2} 6 p^{1} 8 d^{1}, 6 s^{2} 6 p^{1} 9 s^{1}\end{array}$ & 63 & 179 & 179 \\
\hline
\end{tabular}


Table B6. Summary of HULLAC calculations. The last column shows the number of transitions whose upper level is below the ionization potential.

\begin{tabular}{|c|c|c|c|c|}
\hline Ion & Configurations & $N_{\text {level }}$ & $N_{\text {line }}$ & $N_{\text {line }}^{*}$ \\
\hline Rn I & $\begin{array}{l}\mathbf{6} \mathbf{p}^{6}, 6 p^{5} 7 s^{1}, 6 p^{5} 7 p^{1}, 6 p^{5} 6 d^{1}, 6 p^{5} 8 p^{1} \\
6 p^{5} 7 d^{1}, 6 p^{5} 9 s^{1}, 6 p^{5} 5 f^{1}\end{array}$ & 65 & 634 & 0 \\
\hline Rn II & $\begin{array}{l}\mathbf{6 s}^{2} \mathbf{6} \mathbf{p}^{\mathbf{5}}, 6 s^{1} 6 p^{6}, 6 s^{2} 6 p^{4} 7 s^{1}, 6 s^{2} 6 p^{4} 6 d^{1}, 6 s^{2} 6 p^{4} 7 p^{1} \\
6 s^{2} 6 p^{4} 8 s^{1}, 6 s^{2} 6 p^{4} 7 d^{1}, 6 s^{2} 6 p^{4} 5 f^{1}, 6 s^{2} 6 p^{4} 8 p^{1}, 6 s^{2} 6 p^{4} 9 s^{1}\end{array}$ & 155 & 3685 & 477 \\
\hline Rn III & $\begin{array}{l}\mathbf{6 s}^{2} \mathbf{6} \mathbf{p}^{\mathbf{4}}, 6 s^{1} 6 p^{5}, 6 s^{2} 6 p^{3} 6 d^{1}, 6 s^{2} 6 p^{3} 7 s^{1}, 6 s^{2} 6 p^{3} 7 p^{1} \\
6 s^{2} 6 p^{3} 5 f^{1}, 6 s^{2} 6 p^{3} 7 d^{1}, 6 s^{2} 6 p^{3} 8 s^{1}, 6 s^{2} 6 p^{3} 6 f^{1}, 6 p^{6}\end{array}$ & 214 & 6052 & 1336 \\
\hline Rn IV & $\begin{array}{l}\mathbf{6 s}^{\mathbf{2}} \mathbf{6} \mathbf{p}^{3}, 6 s^{1} 6 p^{4}, 6 s^{2} 6 p^{2} 6 d^{1}, 6 s^{2} 6 p^{2} 7 s^{1}, 6 s^{2} 6 p^{2} 5 f^{1} \\
6 s^{2} 6 p^{2} 7 p^{1}\end{array}$ & 100 & 1480 & 1368 \\
\hline Fr I & $\begin{array}{l}\mathbf{6} \mathbf{p}^{6} 7 \mathbf{s}^{1}, 6 p^{6} 7 p^{1}, 6 p^{6} 6 d^{1}, 6 p^{6} 8 s^{1}, 6 p^{6} 8 p^{1} \\
6 p^{6} 7 d^{1}, 6 p^{6} 9 s^{1}, 6 p^{6} 9 p^{1}, 6 p^{6} 8 d^{1}, 6 p^{6} 10 s^{1} \\
\mathbf{6 p}^{6}, 6 p^{5} 7 s^{1}, 6 p^{5} 6 d^{1}, 6 p^{5} 7 p^{1}, 6 p^{5} 8 s^{1}\end{array}$ & 16 & 51 & 18 \\
\hline Fr II & $\begin{array}{l}6 p^{5} 7 d^{1}, 6 p^{5} 5 f^{1}, 6 p^{5} 8 p^{1}, 6 p^{5} 9 s^{1}, 6 p^{5} 6 f^{1} \\
6 p^{5} 8 d^{1}, 6 p^{5} 9 p^{1} \\
\mathbf{6 s}^{2} \mathbf{6} \mathbf{p}^{5}, 6 s^{1} 6 p^{6}, 6 s^{2} 6 p^{4} 6 d^{1}, 6 s^{2} 6 p^{4} 7 s^{1}, 6 s^{2} 6 p^{4} 7 p^{1}\end{array}$ & 103 & 1569 & 132 \\
\hline Fr III & $\begin{array}{l}6 s^{2} 6 p^{4} 5 f^{1}, 6 s^{2} 6 p^{4} 7 d^{1}, 6 s^{2} 6 p^{4} 8 s^{1}, 6 s^{2} 6 p^{4} 8 p^{1}, 6 s^{2} 6 p^{4} 6 f^{1}, \\
6 s^{2} 6 p^{4} 9 s^{1}\end{array}$ & 185 & 4932 & 1231 \\
\hline Fr IV & $\begin{array}{l}\mathbf{6 s}^{2} \mathbf{6} \mathbf{p}^{4}, 6 s^{1} 6 p^{5}, 6 s^{2} 6 p^{3} 6 d^{1}, 6 s^{2} 6 p^{3} 7 s^{1}, 6 s^{2} 6 p^{3} 7 d^{1} \\
6 s^{2} 6 p^{3} 8 s^{1}, 6 s^{2} 6 p^{3} 8 d^{1}, 6 s^{2} 6 p^{3} 9 s^{1}\end{array}$ & 153 & 410 & 307 \\
\hline Ra I & $\begin{array}{l}7 \mathbf{s}^{2}, 7 \mathbf{s}^{1} 8 \mathbf{s}^{1}, 7 \mathbf{s}^{1} 9 \mathbf{s}^{1}, 7 s^{1} 7 p^{1}, 7 s^{1} 6 d^{1} \\
6 d^{1} 7 p^{1}, 7 s^{1} 8 p^{1}, 7 p^{2}, 7 s^{1} 7 d^{1}, 7 s^{1} 5 f^{1} \\
7 s^{1} 6 f^{1}, 8 s^{1} 7 p^{1}, 8 s^{1} 8 p^{1}, 9 s^{1} 7 p^{1}, 9 s^{1} 8 p^{1} \\
6 d^{1} 8 p^{1}, 7 d^{1} 7 p^{1}, 7 d^{1} 8 p^{1}, 7 p^{1} 8 p^{1}, 7 s^{1} 8 d^{1} \\
8 s^{1} 6 d^{1}, 8 s^{1} 7 d^{1}, 8 s^{1} 8 d^{1}, 9 s^{1} 6 d^{1}, 9 s^{1} 7 d^{1} \\
9 s^{1} 8 d^{1}\end{array}$ & 112 & 2024 & 75 \\
\hline Ra II & $\begin{array}{l}\mathbf{6 p}^{6} 7 \mathbf{s}^{1}, 6 p^{6} 8 s^{1}, 6 p^{6} 9 s^{1}, 6 p^{6} 6 d^{1}, 6 p^{6} 7 d^{1} \\
6 p^{6} 8 d^{1}, 6 p^{6} 7 p^{1}, 6 p^{6} 8 p^{1}, 6 p^{6} 5 f^{1}, 6 p^{6} 6 f^{1} \\
\mathbf{6 p}^{6}, 6 p^{5} 6 d^{1}, 6 p^{5} 7 s^{1}, 6 p^{5} 5 f^{1}, 6 p^{5} 7 p^{1}\end{array}$ & 17 & 48 & 7 \\
\hline Ra III & $\begin{array}{l}6 p^{5} 7 d^{1}, 6 p^{5} 8 s^{1}, 6 p^{5} 6 f^{1}, 6 p^{5} 8 p^{1}, 6 p^{5} 8 d^{1} \\
6 p^{5} 9 s^{1}\end{array}$ & 93 & 1251 & 252 \\
\hline Ra IV & $\mathbf{6 s}^{2} \mathbf{6} \mathbf{p}^{\mathbf{5}}, 6 s^{1} 6 p^{6}, 6 s^{2} 6 p^{4} 6 d^{1}, 6 s^{2} 6 p^{4} 7 s^{1}, 6 s^{2} 6 p^{4} 7 p^{1}$ & 60 & 574 & 457 \\
\hline
\end{tabular}



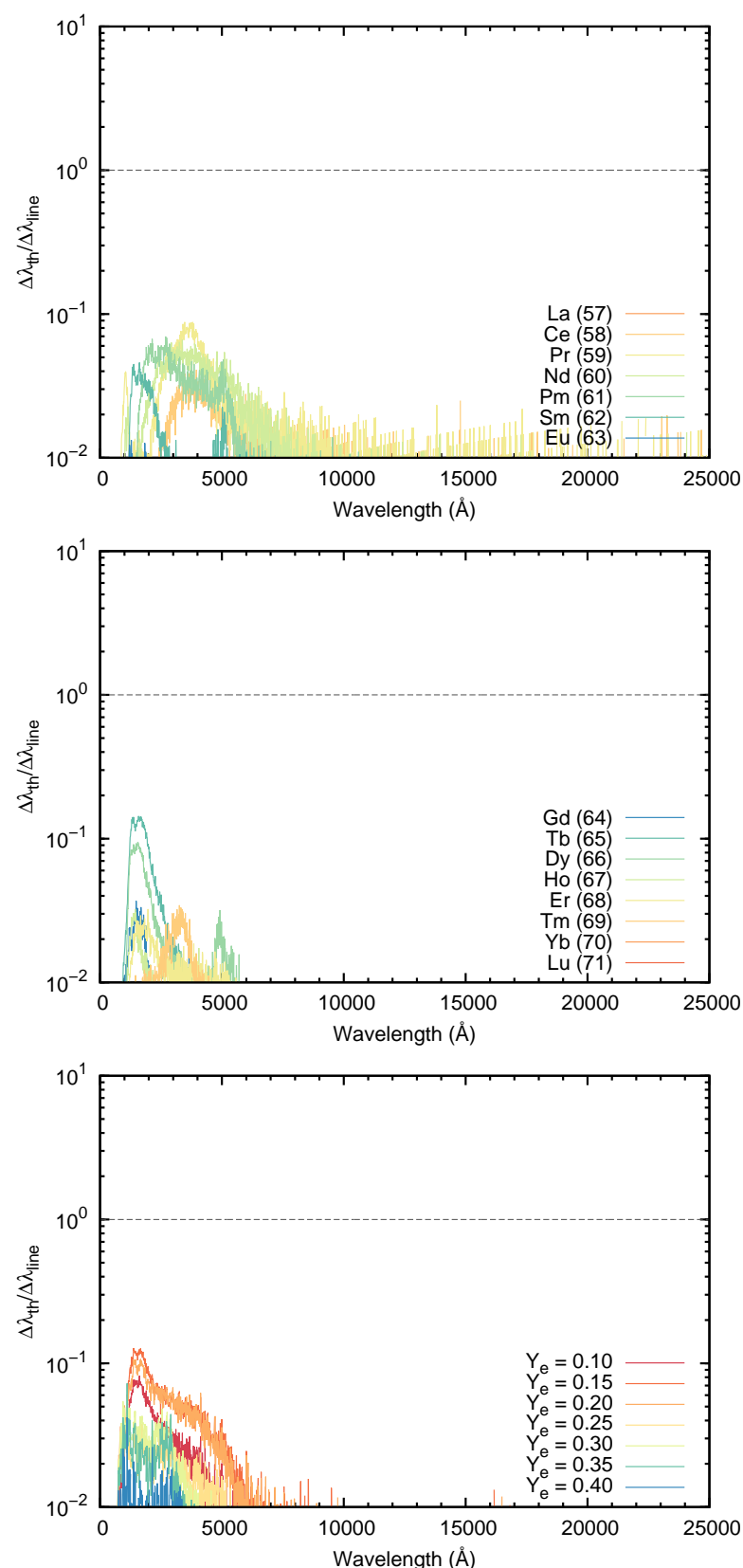

Figure A3. The ratio of the thermal width of the line $\Delta \lambda_{\text {th }}$ to the typical wavelength spacing of the transitions $\Delta \lambda_{\text {line }}$ for the ejecta with $\rho=1 \times 10^{-13} \mathrm{~g} \mathrm{~cm}^{-3}$ and $T=5000 \mathrm{~K}$ at $t=1$ day after the merger. The line spacing is evaluated for the strong lines with $\tau_{l}>1$. Top and middle panels show the ratio for singleelement ejecta (lanthanide) and the bottom panel shows the same for the mixture of the elements. For the mixture of the elements, the thermal width is calculated with the averaged mass number. Below the dashed line, the condition of $\Delta \lambda_{\text {line }}>\Delta \lambda_{\text {th }}$ is satisfied. 